\title{
The role of SNAP29 during epidermal differentiation
}

\author{
Doctoral Thesis
}

\author{
In partial fulfillment of the requirements for the degree \\ "Doctor rerum naturalium (Dr. rer. nat.)" \\ in the Molecular Medicine Study Program \\ at the Georg-August University Göttingen
}

submitted by

\section{Christina Seebode}

born in Göttingen, Germany

Göttingen, 28.06.2015 


\section{Members of the thesis committee}

\section{Supervisors:}

Prof. Dr. med. Steffen Emmert and Dr. Stina Schiller

University Medical Center, Dept. of Dermatology, Venereology and Allergology

Georg-August University Göttingen

First member of the thesis committee:

Prof. Dr. med. Michael P. Schön

University Medical Center, Dept. of Dermatology, Venereology and Allergology

Georg-August University Göttingen

Second member of the thesis committee:

Prof. Dr. Jürgen Brockmöller

University Medical Center, Dept. of Clinical Pharmacology

Georg-August University Göttingen

Third member of the thesis committee:

Prof. Dr. med. Heidi Hahn

Department for Human Genetics, Section of Developmental Genetics

Georg-August University Göttingen

Date of Disputation: 


\section{AFFIDAVIT}

Here I declare that my doctoral thesis entitled

"The role of SNAP29 during epidermal differentiation"

has been written independently with no other sources and aids than quoted.

Date

Signature (Christina Seebode) 
Firstly, I would like to thank my supervisor Prof. Dr. med. Steffen Emmert for giving me the opportunity to write this thesis in his lab and for all the successful publications during this time. I am very thankful for his inspiring guidance, and his constructive and critical support for this thesis project. Furthermore, I owe thanks to Dr. Stina Schiller for her advice and support as my thesis supervisor. She was always available to discuss my research and willing to help with even the smallest of problems.

I also want to acknowledge the members of my Thesis Committee, Prof. Dr. med. Michael P. Schön, Prof. Dr. Jürgen Brockmöller and Prof. Dr. med. Heidi Hahn for their advice regarding research and academic life.

I would like to thank Thorben Ruhwedel at the Max Planck Institute for Experimental Medicine, Department of Neurogenetics, who provided technical support regarding the ultrastructural analyses.

Moreover, I would like to thank all my lab colleagues for their contribution and encouragement during the three years: Dr. Steffen Schubert, Regina Tiede, Petra Laspe, and Sabine Smolorz. Special thanks go to Antje Apel und Janin Lehmann for the great and continuous support during every day work regarding scientific expertise and practical support. I also thank Anette Bennemann for technical support regarding tissue preparation and histology.

I want like to thank all my friends and colleagues in Göttingen for their support during the development of this thesis

Finally, I want to thank my parents for their encouragement and support during the entire course of my $\mathrm{PhD}$. 


\section{List of Publications}

(1) Schäfer A., Schubert S., Gratchev A., Seebode C., Apel A., Laspe P., Hofmann L., Ohlenbusch A., Mori T., Kobayashi N., Schürer A., Schön M.P., Emmert S.; Characterization of 3 XPG-defective patients identifies 3 missense mutations that impair repair and transcription. Journal of Investigative Dermatology, 2013; 133, $1841-1849$.

(2) Schäfer A., Gratchev A., Seebode C., Hofmann L., Schubert S., Laspe P., Apel A., Ohlenbusch A., Tzvetkov M., Weishaupt C., Oji V., Schön MP., Emmert S.; Functional and molecular genetic analyses of 9 newly identified XPD-deficient patients reveal a novel mutation resulting in TTD as well as in XP/CS complex phenotypes. Experimental Dermatology, 2013; 22, 486 - 489.

(3) Schiller S.*, Seebode C.*, Hennies HC., Giehl K., Emmert S.; Palmoplantar keratoderma (PPK): aquired and genetic causes of a not so rare disease. Journal of the German Society of Dermatology, 2014; 12, 781-8 (*contributed equally to this work).

(4) Seebode C.*, Schiller S.*, Emmert S., Giehl K.; Palmoplantar dermatoses: when should genes be considered? Der Hautarzt, 2014; 65: 499-512 (*contributed equally to this work).

(5) Schiller S.*, Seebode C.* Wieser GL., Goebbels S., Moebius W., Horowitz M., Sarig O., Sprecher E., Emmert S. Establishment of two mouse models for CEDNIK syndrome reveals the pivotal role of SNAP29 in epidermal differentiation. Journal of Investigative Dermatology, 2015; in press (*contributed equally to this work). 


\section{Table of contents}

List of Publications........................................................................................... III

List of Tables................................................................................................. VIII

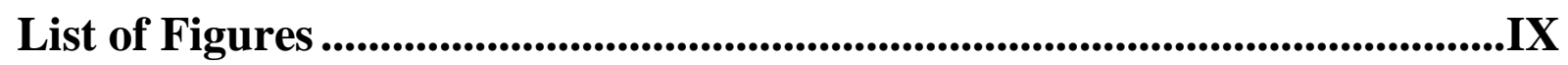

Abstract .........................................................................................................................

List of Abbreviations.................................................................................................. XII

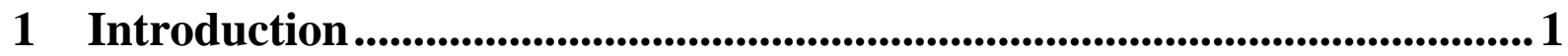

1.1 Structure and function of the skin................................................................ 1

1.2 The epidermis and terminal differentiation of keratinocytes.................................. 1

1.2.1 Formation of an intracellular keratin network ................................................. 3

1.2.2 Formation of the cornified envelope (CE) …............................................. 5

1.2.3 The formation of lipid lamellae ............................................................... 5

1.2.4 Lamellar bodies (LBs) and barrier homeostasis........................................... 6

1.2.5 Desquamation, the shedding of corneocytes ............................................ 7

1.2.6 Epidermal homeostasis and associated skin diseases ................................. 8

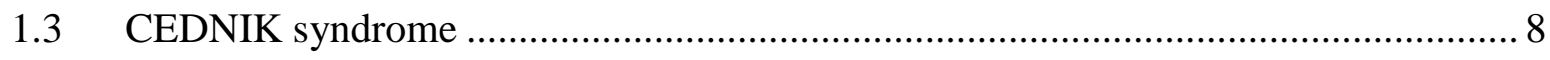

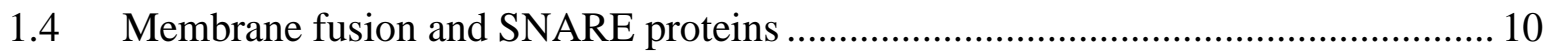

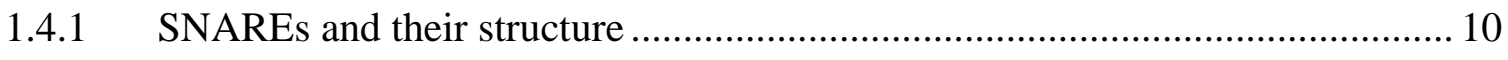

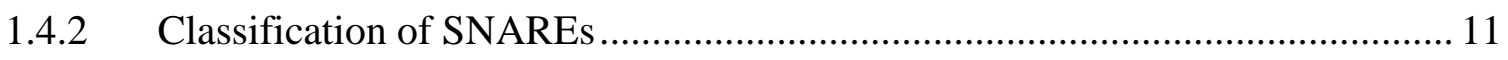

1.4.3 SNARE core complex formation and dissasembly .................................... 12

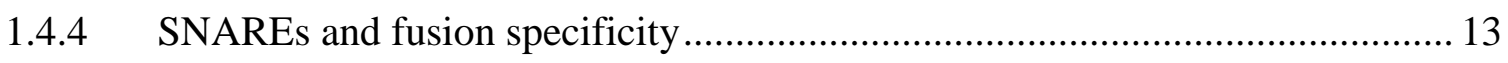

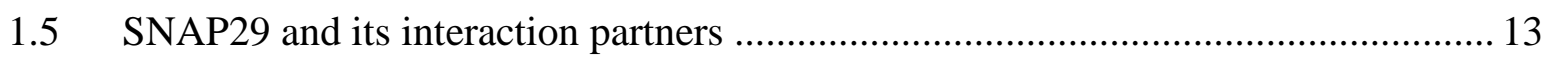

1.5.1 SNAP29 in membrane fusion events ..................................................... 14

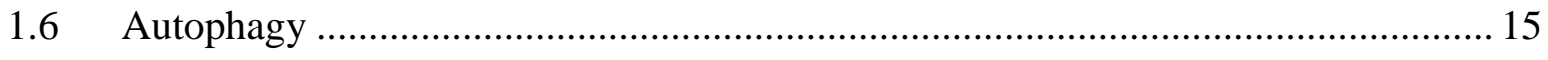

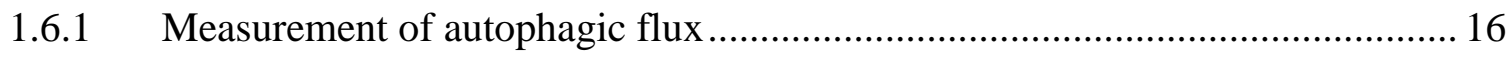

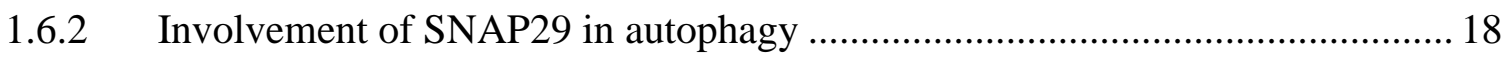

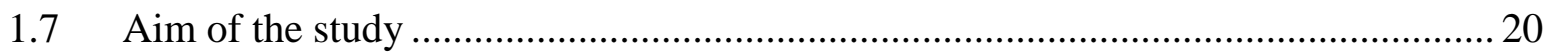

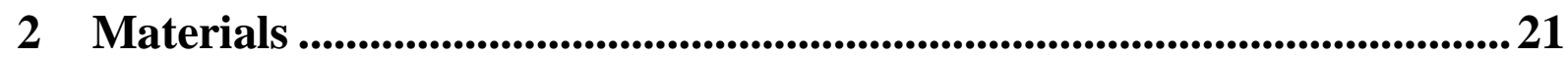




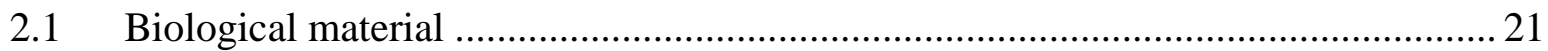

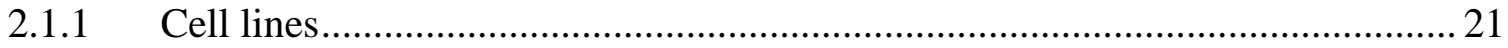

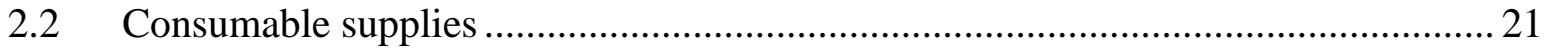

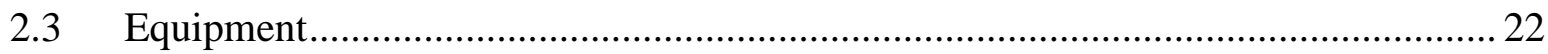

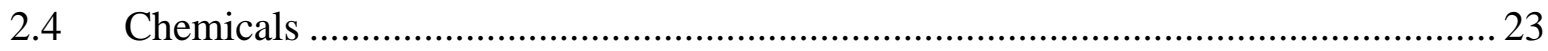

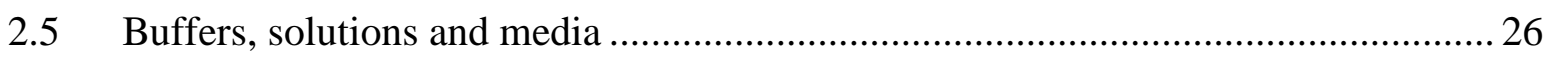

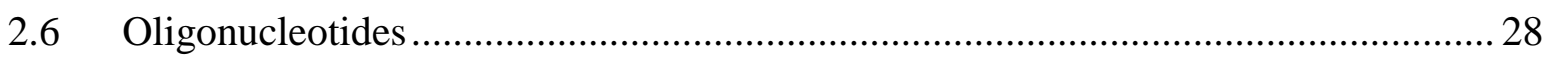

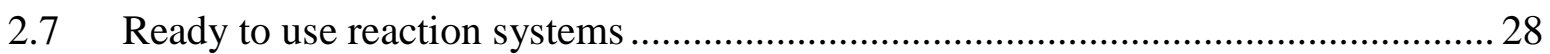

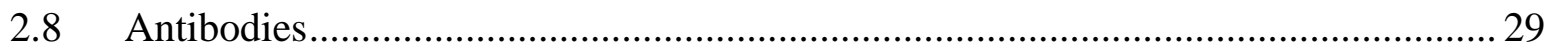

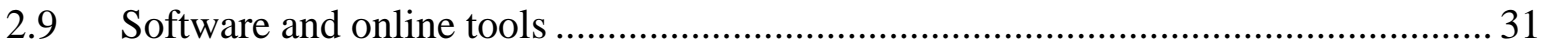

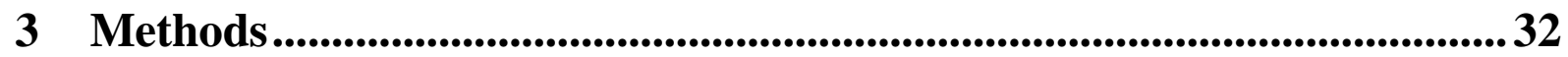

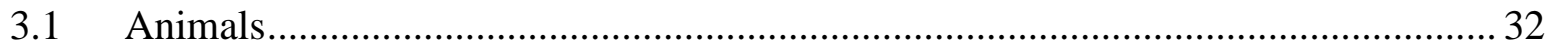

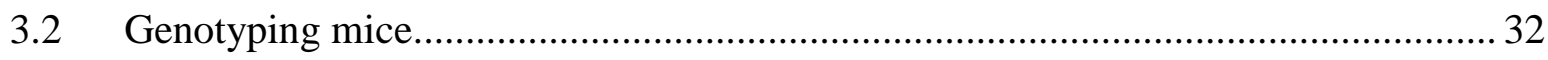

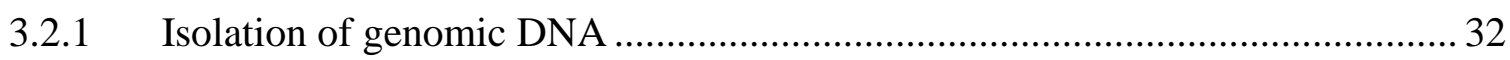

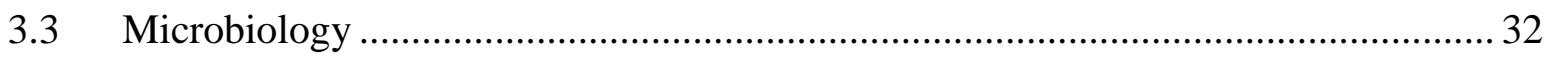

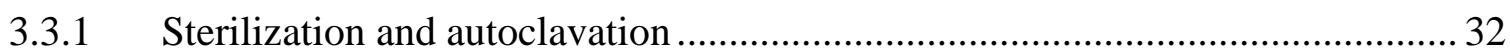

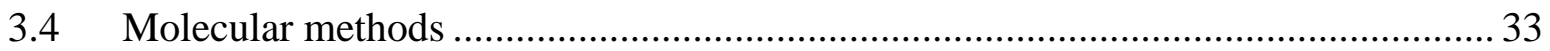

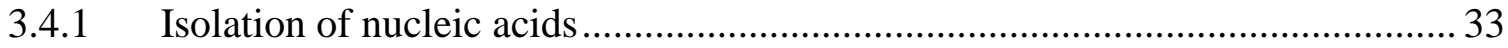

3.4.1.1 Extraction of nucleic acids from agarose gels ........................................ 33

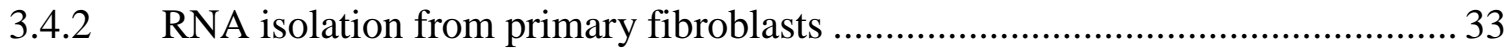

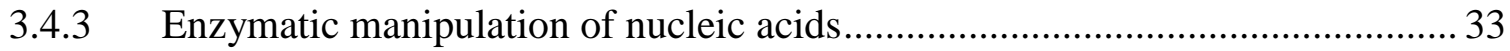

3.4.3.1 Complementary DNA (cDNA) synthesis ............................................ 33

3.4.4 Polymerase Chain Reaction (PCR) ….......................................................... 34

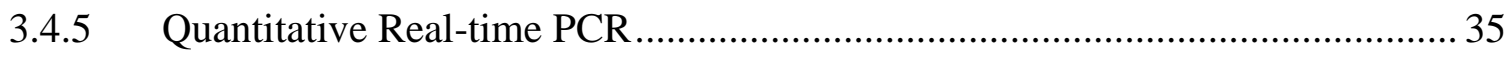

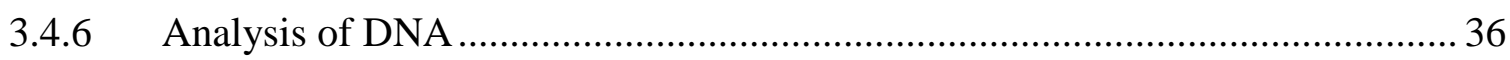

3.4.6.1 Agarose gel electrophoresis (AGE) …................................................ 36

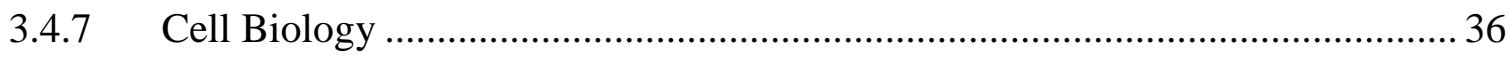

3.4.7.1 Preparation of primary murine fibroblasts ........................................... 36 


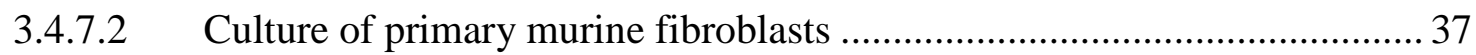

3.4.7.3 Isolation of primary murine keratinocytes .......................................... 37

3.4.7.4 Cultivation of primary murine keratinocytes ............................................... 37

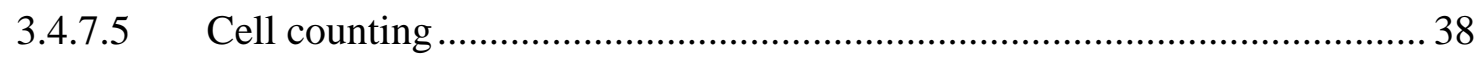

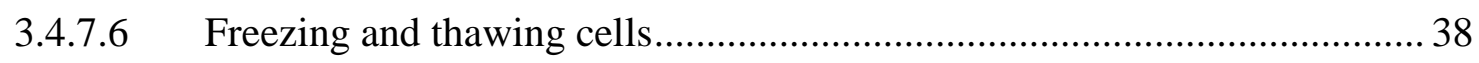

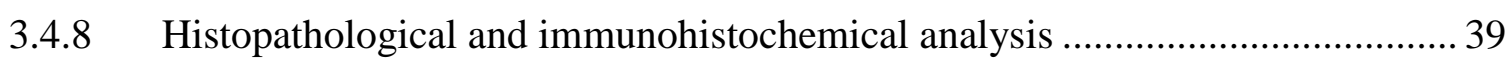

3.4.8.1 Tissue preparation, paraffin and OCT embedding, sectioning .................. 39

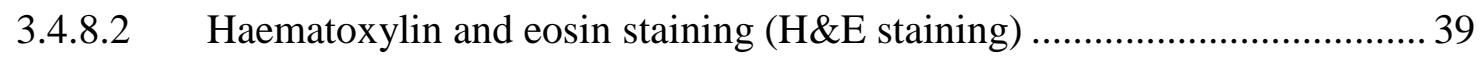

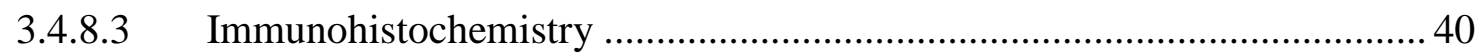

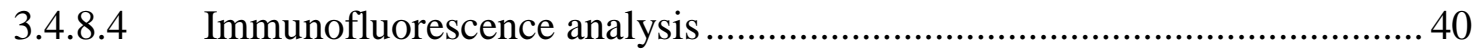

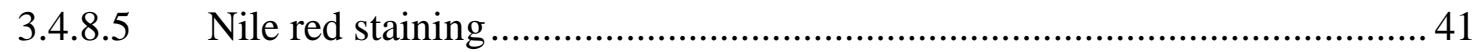

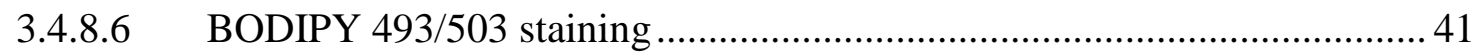

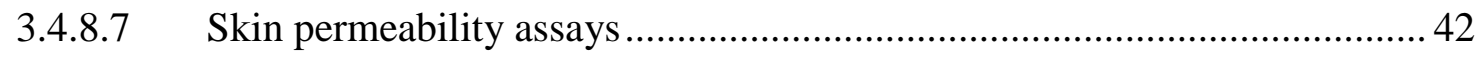

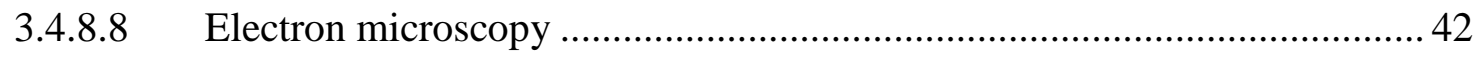

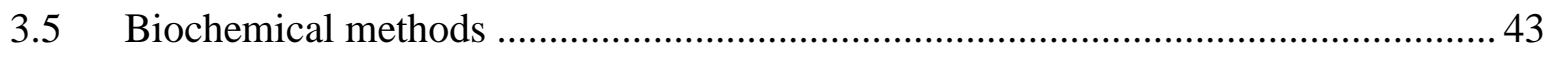

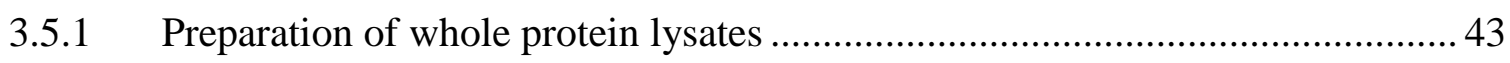

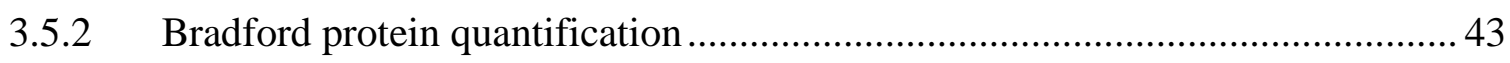

3.5.3 Polyacrylamide gel electrophoresis (SDS-PAGE) ........................................ 43

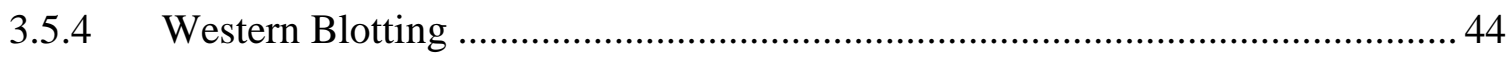

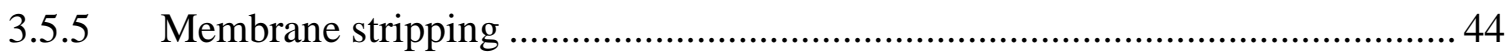

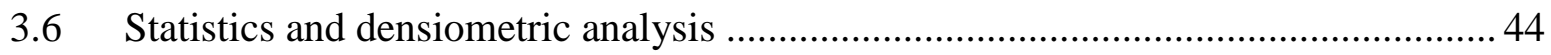

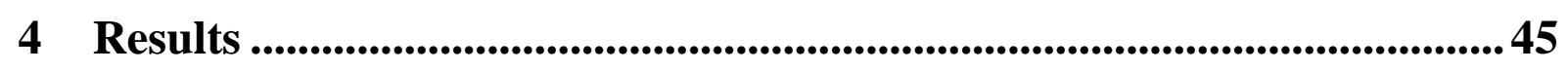

4.1 Generation of total or epidermal Snap29-deficient mice..................................... 45

4.2 Confirmation of a functional Snap29 knockout ............................................... 49

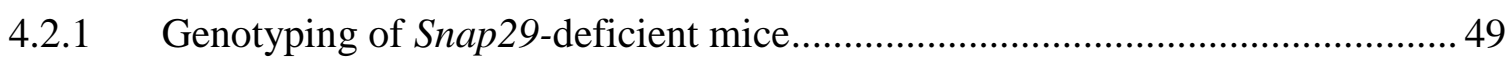

4.2.2 Quantitative Real-time PCR, Western Blot, and immunohistochemical staining.. 
4.3 Snap29 deficient mice exhibit a distinct ichthyotic phenotype and neonatal lethality..

4.4 Snap29 deficiency causes altered histomorphological features in mouse skin.........54

4.5 Keratinocyte differentiation is disturbed in mice lacking SNAP29.......................56

4.6 Snap29 deficiency causes enhanced levels of epidermal proliferation. .................... 60

4.7 Ultrastructural analysis of Snap29-deficient epidermis reveals altered formation and maturation of LBs.

4.8 Snap29-deficient keratinocytes exhibit disturbed secretion of LB contents. ............ 64

4.9 Snap29-deficient newborns show a defective epidermal lipid distribution............... 65

4.10 SNAP29 deficiency causes impaired barrier permeability ....................................... 66

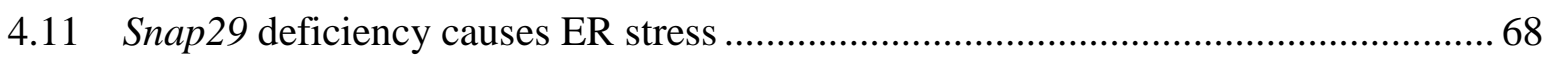

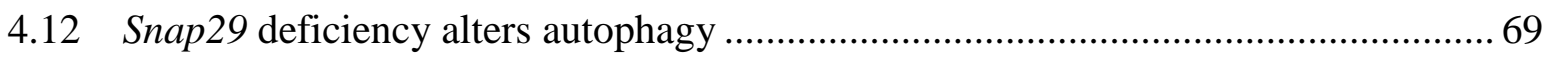

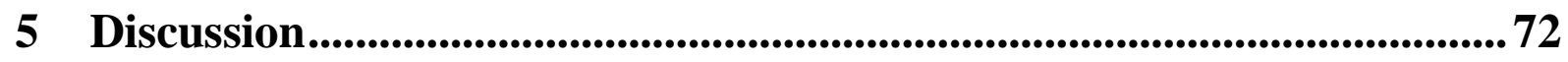

5.1 Generation of two Snap29-deficient mouse models ............................................. 72

5.2 Snap29-deficient mice mimic the human CEDNIK syndrome phenotype................ 73

5.3 SNAP29 is essential for a functional epidermal barrier. ...................................... 74

5.4 Snap29 deficiency provokes a disturbed LB appearance .................................... 75

5.5 Snap29 deficiency alters the epidermal lipid structure........................................ 76

5.6 Increased proliferation and abnormal keratinocyte differentiation in Snap29-deficient

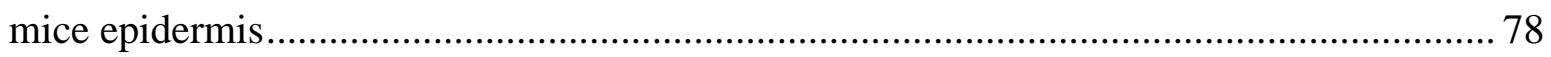

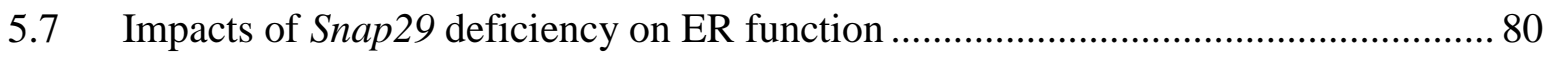

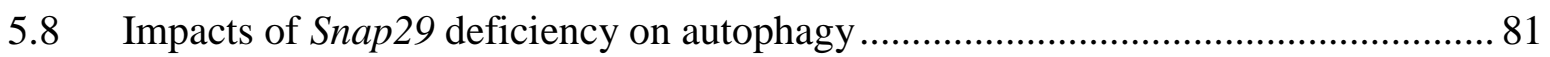

5.9 Total knockout mice show a more pronounced phenotype …................................. 84

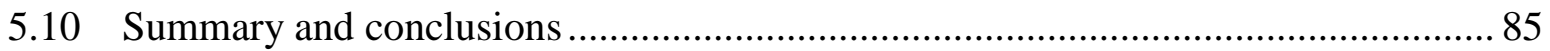

6 Bibliography (........................................................................................................86

7 Curriculum vitae ......................................................................................100 


\section{List of Tables}

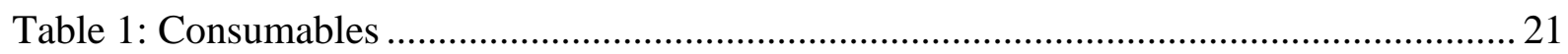

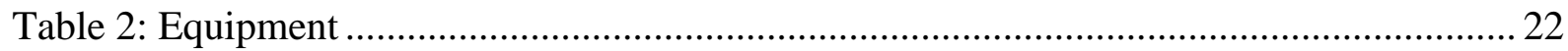

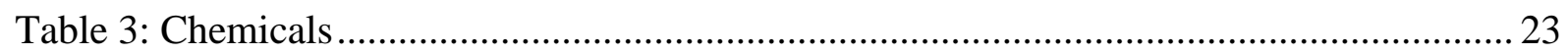

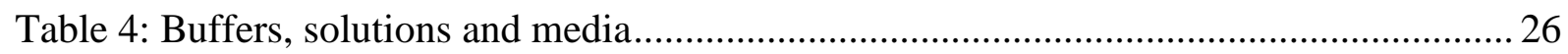

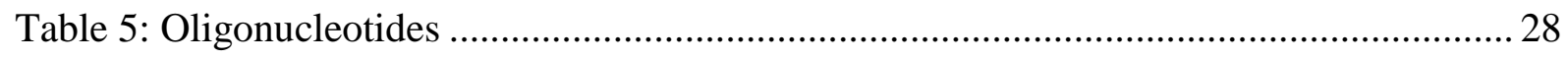

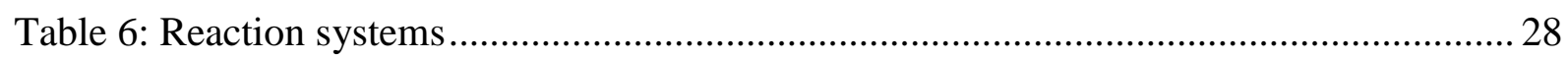

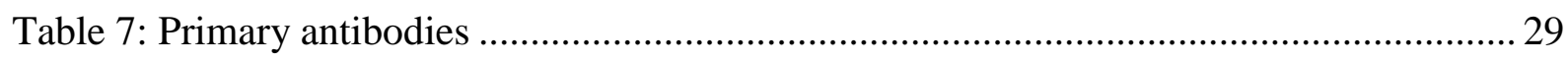

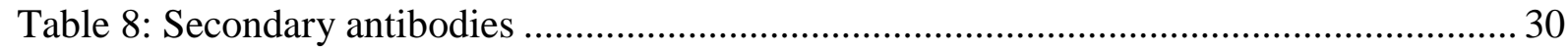

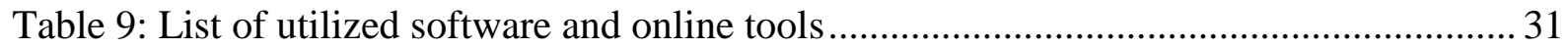




\section{List of Figures}

Figure 1: Schematic illustration of the epidermis in mammals............................................. 2

Figure 2: Schematic illustration of the multi-layered epidermis.......................................... 4

Figure 3: Schematic illustration of formation and maturation of LBs. ................................... 7

Figure 4: Cutaneous phenotype of CEDNIK syndrome patients ......................................... 9

Figure 5: Schematic domain structure of SNAREs......................................................... 12

Figure 6: Schematic illustration of the assembly of the acceptor complex (Qabc-SNARE

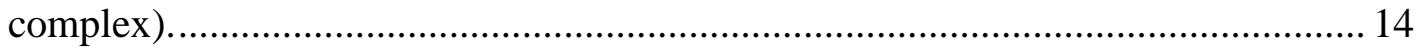

Figure 7: Incorporation of LC3-II and p62 into the autophagosome.................................. 17

Figure 8: Regulation of autophagosome-lysosome fusion ................................................ 19

Figure 9: Alignments of murine SNAP29 with its human ortholog revealed 83\% amino

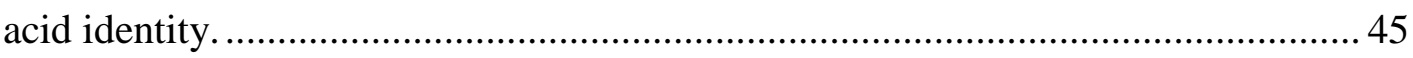

Figure 10: Schematic illustration of the generation of Snap29-deficient mice....................... 47

Figure 11: Allele constructs and strategies used for the generation of Snap29-/- and

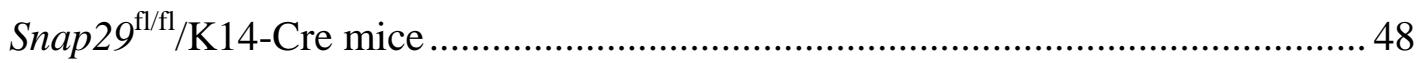

Figure 12: Representative PCR products of genomic DNA isolated from tail biopsies

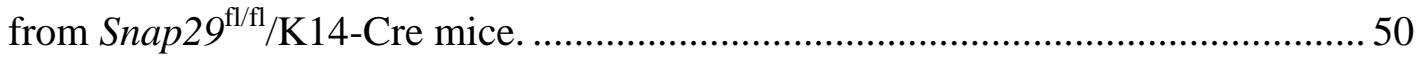

Figure 13: Snap29 ablation in fibroblasts derived from Snap $29^{-/-}$mice. .................................. 50

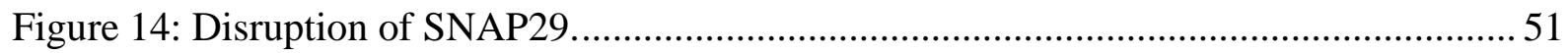

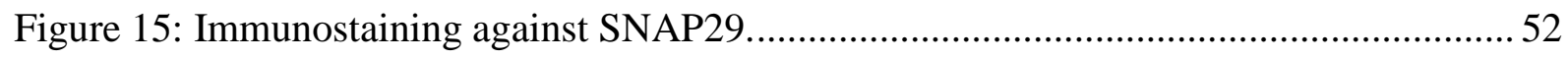

Figure 16: Immunofluorescence staining against SNAP29 in keratinocytes...........................53

Figure 17: Ichthyotic phenotype of Snap29-deficient mice.................................................5 54

Figure 18: Comparison of skin morphology of Snap29-deficient and WT mice.....................56

Figure 19: Analysis of early and late differentiation markers............................................. 57

Figure 20: Immunofluorescence double staining with early and late differentiation

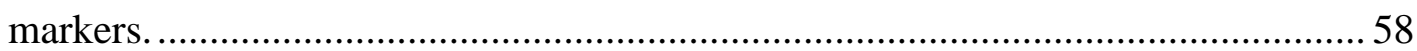

Figure 21: Analysis of late differentiation markers. .......................................................... 59

Figure 22: Hyperproliferative conditions in Snap29-deficient mice....................................... 61

Figure 23: No detection of apoptotic activity in Snap29-deficient mice. ............................... 61

Figure 24: Ultrastructural analysis of control (a/d/g), Snap29 $9^{\mathrm{fl} / \mathrm{fl}} / \mathrm{K} 14-\mathrm{Cre}(\mathrm{b} / \mathrm{e} / \mathrm{h})$, and Snap $29^{-/-}(\mathrm{c} / \mathrm{f} / \mathrm{i})$ epidermis. 63

Figure 25: Ultrastructural variations between total and epidermal knockout mice. ................ 64

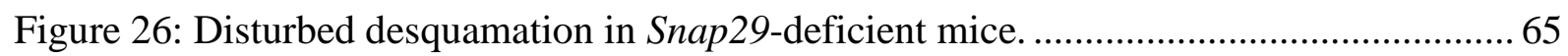

Figure 27: Defective epidermal lipid distribution in Snap29-deficient skin........................... 66 


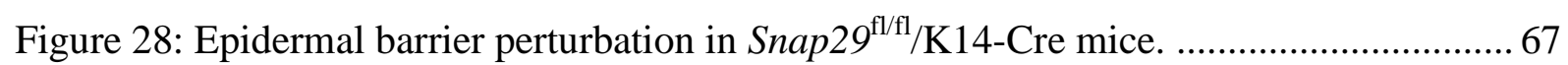

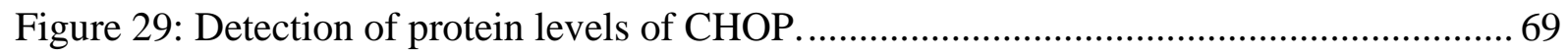

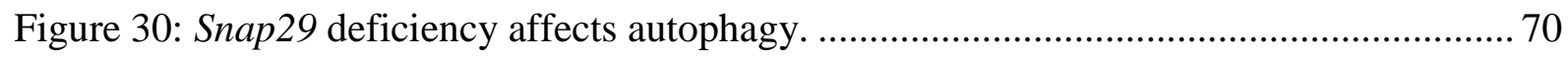

Figure 31: Effects of bafilomycin A1 on LC3B-II levels. ................................................. 71 


\section{Abstract}

The human CEDNIK syndrome is caused by loss-of-function mutations in the SNAP29 gene, encoding a member of the SNARE family of proteins. So far, no Snap29-deficient mammalian animal models have been described for this rare neurocutaneous syndrome and this work reports the generation and characterization of a total $\left(\right.$ Snap29 $\left.{ }^{-1-}\right)$ as well as keratinocyte specific (Snap29 $\left.9^{\mathrm{fl} / \mathrm{fl}} / \mathrm{K} 14-\mathrm{Cre}\right)$ Snap29 knockout mouse line. Both Snap29deficient mouse models developed an ichthyotic phenotype closely resembling the human epidermal CEDNIK syndrome phenotype. Thus, both lines can be used as beneficial models to further enlighten the unique impact of SNAP29 in epidermal differentiation as well as in understanding the pathophysiological mechanisms in common epidermal differentiation disorders and they provide a deeper insight into the molecular basis of the pathogenesis of CEDNIK syndrome.

Using immunohistochemistry, SNAP29 deficiency was found to result in acanthosis and hyperkeratosis as well as in abnormal keratinocyte differentiation with increased proliferation in both mutant mouse lines. In addition, Snap29-deficient mice revealed a severely impaired cutaneous permeability barrier function accompanied by neonatal lethality. These results indicate an essential role of SNAP29 in epidermal differentiation and barrier formation. Furthermore, ultrastructural analyses revealed malformed LBs and markedly decreased deposition of LB contents in mutant mice epidermis indicating a severe impairment in LB function in the epidermis due to the Snap29 knockout. Thus, SNAP29 is essential for LB maturation and secretion as demonstrated by abnormal lipid profiles, and an impaired barrier formation, which are most likely due to an impaired vesicular trafficking.

In addition to the impact on terminal keratinocyte differentiation, ablation of SNAP29 affects ER stress and autophagy. By western blot analyses, SNAP29 ablation was found to result in a strong induction of the ER stress marker CHOP (C/EBP homologous protein) in mutant mice and the autophagosomal marker LC3B-II was increased under starvation and bafilomycin A1 treatment, whereas p62/SQSTM1 protein amounts remained unchanged.

In conclusion, this work contributes to the substantial role of SNAP29 in epidermal differentiation, particularly in LB formation and maturation. SNAP29 is also involved in the complex machinery of autophagy. 


\section{List of Abbreviations}

\begin{tabular}{|c|c|}
\hline${ }^{\circ} \mathrm{C}$ & degree Celsius \\
\hline$\mu g$ & micro gram \\
\hline APS & ammonium persulfate \\
\hline ARC & Arthrogryposis-Renal dysfunction-Cholestasis \\
\hline Atg & autophagy-related \\
\hline ATP & adenosine triphosphate \\
\hline bidest & double distilled water \\
\hline $\mathrm{CaCl}_{2}$ & calcium chloride \\
\hline $\mathrm{CE}$ & cornified envelope \\
\hline cDNA & complementary DNA \\
\hline CDSN & corneodesmosin \\
\hline CEDNIK & $\begin{array}{l}\text { CErebral Dysgenesis, Neuropathy, Ichthyosis and } \\
\text { Keratoderma }\end{array}$ \\
\hline COPI & coat protein complex I \\
\hline COPII & coat protein complex II \\
\hline CRAMP & cathelicidin-related anti-microbial peptide \\
\hline $\mathrm{dH}_{2} \mathrm{O}$ & distilled water \\
\hline DMEM & Dulbecco's modified Eagle's medium \\
\hline DMSO & dimethyl sulfoxide \\
\hline DNA & deoxyribonucleic acid \\
\hline dNTP & deoxynucleoside triphosphates \\
\hline EDTA & ethylenediaminetetraacetic acid \\
\hline e.g. & for example \\
\hline EHD1 & EH domain-containing protein 1 \\
\hline EM & electron microscopy \\
\hline EMMA & European Mouse Mutant Archive \\
\hline EST & Estland \\
\hline $\mathrm{EtOH}$ & ethanol \\
\hline FBS & fetal bovine serum \\
\hline FITC & fluoresceinisothiocyanate \\
\hline $\mathrm{fl}$ & floxed \\
\hline for & forward \\
\hline GER & Germany \\
\hline $\mathrm{H} \& \mathrm{E}$ & hematoxylin and eosin \\
\hline HBSS & Hank's Balanced Salt Solution \\
\hline HRP & horseradish peroxidase \\
\hline $\operatorname{hr}(\mathrm{s})$ & hour(s) \\
\hline K1 & Keratin 1 \\
\hline
\end{tabular}


K5

K6

K10

K14

K16

$\mathrm{kDa}$

KLK5

KLK7

LEKTI

LC3

$\mathrm{M}$

$\mathrm{m} / \mathrm{v}$

MEDNIK

mer

MEF

$\mathrm{MgCl}_{2}$

MGP

$\min$

$\mathrm{mM}$

$\mathrm{mm}$

$\mathrm{NaCl}$

$\mathrm{NaHCO}_{3}$

$\mathrm{NaOH}$

$\mathrm{NMF}$

NPF

$\mathrm{NSF}$

$O$-GlcNAc

OGT

$\mathrm{o} / \mathrm{n}$

$\mathrm{OD}_{\mathrm{xxnm}}$

oligo

PAGE

PBS

PCR

PE

PFA

$\mathrm{pH}$

$\mathrm{P} / \mathrm{S}$

rev
Keratin 5

Keratin 6

Keratin 10

Keratin 14

Keratin 16

kilo Dalton

kallikrein 5

kallikrein7

lympho-epithelial Kazal-type related inhibitor

microtubule-associated protein light chain 3

molar

mass/volume

Mental retardation, Enteropathy, Deafness, Neuropathy, Ichthyosis, and Keratoderma

repeat unit

mouse embryonic fibroblast

magnesium chloride

Mouse Genetics Project

minute(s)

milli molar

millimeters

sodium chloride

sodium bicarbonate

sodium hydroxide

natural moisturizing factor

asparigine-proline-phenylalanine

$\mathrm{N}$-ethylmaleimide-sensitive factor

$O$-linked $\beta$ - $N$-acetylglucosamine

$O$-linked $\beta$ - $N$-acetylglucosamine transferase

over night

optical density at xxnm

oligonucleotide

polyacrylamide gel electrophoresis

phosphate-buffered saline

polymerase chain reaction

phosphatidylethanolamine

paraformaldehyd

potentium hydrogenii

penicillin - streptavidin

reverse 


\begin{tabular}{|c|c|}
\hline RLT buffer & buffer Qiagen \\
\hline RNA & ribonucleic acid \\
\hline $\mathrm{rpm}$ & rounds per minute \\
\hline RT & room temperature \\
\hline $\mathrm{SC}$ & stratum corneum \\
\hline SDS & sodium dodecyl sulfate \\
\hline $\sec$ & seconds \\
\hline SG & stratum granulosum \\
\hline SEM & standard error of the mean \\
\hline SNAP & $\begin{array}{l}\text { soluble n-ethylmaleimide sensitive factor attachment } \\
\text { protein }\end{array}$ \\
\hline SNARE & $\begin{array}{l}\text { soluble n-ethylmaleimide sensitive factor attachment } \\
\text { protein receptor }\end{array}$ \\
\hline TA & transit amplifying \\
\hline Taq & Thermus aquaticus \\
\hline TGase & transglutaminase \\
\hline TGN & trans-Golgi network \\
\hline TEMED & $\mathrm{N}, \mathrm{N}, \mathrm{N}, \mathrm{N}$-tetramethyl-ethane-1,2-diamine \\
\hline Tris & Tris(hydroxymethyl)-aminomethane \\
\hline t-SNARE & target synaptosome-associated protein receptor \\
\hline UK & United Kingdom \\
\hline UMG & University medical center Göttingen \\
\hline USA & United states of America \\
\hline UV & ultraviolet \\
\hline $\mathrm{V}$ & volts \\
\hline VAMP & vesicle-associated membrane protein \\
\hline v-SNARE & vesicle synaptosome-associated protein receptor \\
\hline $\mathrm{v} / \mathrm{v}$ & volume/volume \\
\hline $\mathrm{w} / \mathrm{v}$ & weight/volume \\
\hline wt & wild type \\
\hline WTSI & Wellcome Trust Sanger Institute \\
\hline$\mu l$ & micro liter \\
\hline
\end{tabular}




\section{Introduction}

\subsection{Structure and function of the skin}

Mammalian skin is the largest organ of the integumentary system and it is composed of three compartments: the epidermis, the underlying dermis and the subcutis. The epidermis comprises the outermost layers of the skin and functions as a barrier between the body and the external, often hostile, environment. It is involved in a number of physiological functions, such as the maintenance of epidermal hydration, protection against mechanical and physical assaults, thermoregulation, immune defense, and protection against UV irradiation (reviewed by (Proksch et al, 2008)). In order to maintain these functions, keratinocytes undergo a specialized form of terminal differentiation, thereby forming the epidermal barrier (reviewed by (Eckhart et al, 2013)).

\subsection{The epidermis and terminal differentiation of keratinocytes}

In mammals, the epidermis is a stratified squamous epithelium, which is composed of four cell layers: stratum basale, stratum spinosum, stratum granulosum and a dead cornified layer, the stratum corneum. Keratinocytes represent the predominant cell type of the epidermis, which are tightly interconnected by cell-cell junctions called desmosomes (Green \& Jones, 1996; Samuelov \& Sprecher, 2014). Other cell types within the epidermis are Langerhans cells, melanocytes and Merkel cells (see Figure 1), being accountable for antigenpresentation, production of melanin and sensory response, respectively. The epidermis itself is not vascularized, and therefore, it is provided with all necessary nutrients by the highly vascularized dermis. The basement membrane (lamina basalis) connects and functionally separates the epidermis from the dermis (Behrens et al, 2012). The epithelial cells are connected to the basement membrane via hemidesmoses, which are highly specialized integrin-mediated epithelial attachment structures (Borradori \& Sonnenberg, 1999).

Keratinocytes undergo a continuous process of terminal differentiation, starting from proliferating basal cells, which migrate from the stratum basale, through the transitional layers, and subsequently forming terminal differentiated keratinocytes. These cells are known as corneocytes, which are flattened, enucleated dead cornified cells. This process of terminal keratinocyte differentiation, known as cornification, is well organized, both spatially and temporally. The three main components of this process include the formation of an 
intracellular keratin network, the formation of a cornified envelope (CE), and the formation of lipid lamellae (reviewed by (Matsui \& Amagai, 2015)).

Finally, the most superficial cornified layers are shed off by a process called desquamation. This differentiation process, ensures a constant rejuvenation of the epidermis and consequently, stem cells within the basal layer have a crucial role in maintaining tissue homeostasis (rewieved by (Blanpain \& Fuchs, 2009)). Each stem cell divides asymmetrically to produce one daughter stem cells as well as one daughter transit amplifying (TA) cell, which undergoes a limited number of cell divisions. TA cells detach from the basement membrane, withdraw from the cell cycle and finally commit to terminal differentiation (reviewed by (Fuchs \& Raghavan, 2002)).

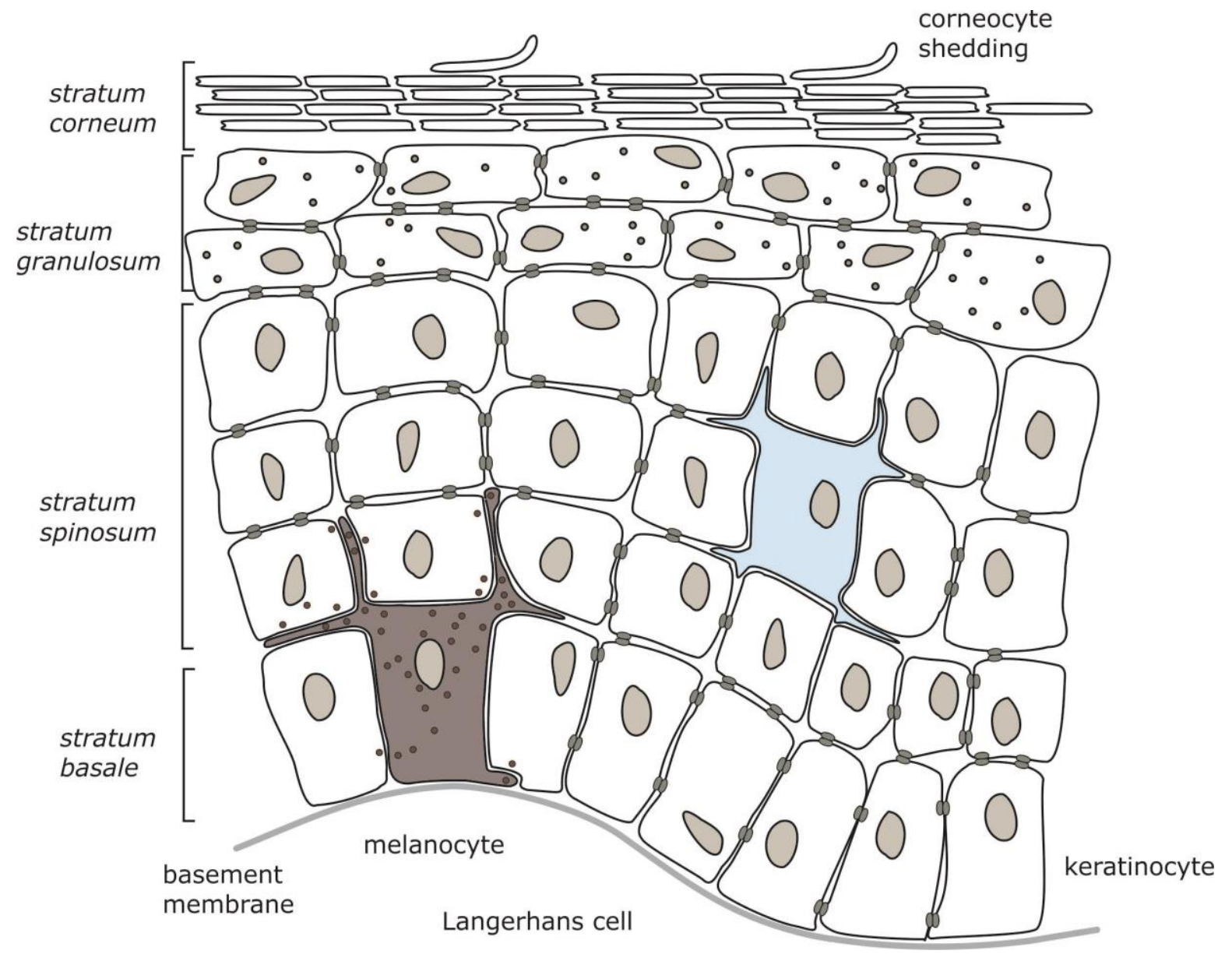

Figure 1: Schematic illustration of the epidermis in mammals.

Keratinocytes are the main cell type in the epidermis. During terminal differentiation they start migrating from the stratum basale, through transitional layers, finally forming the stratum corneum. Other cell types within the epidermis are melanocytes (black), and Langerhans cells (blue), which are pigment-producing and antigen-presenting cells, respectively. Finally, the corneocytes are shed during desquamation. The illustration was kindly provided by Dr. Stina Schiller. 


\subsubsection{Formation of an intracellular keratin network}

The formation of an effective stratum corneum requires the assembly of keratin intermediate filaments. Keratins are the main components of the epithelial cytoskeleton, assembling into intermediate filaments and thereby providing stability to epithelial cells against mechanical stress. In mammalian epidermis keratins are subdivided into type I (acidic) and type II (neutral-basic) keratins, which typically assemble into heterodimers between type I and type II proteins (Fuchs, 1995; Moll et al, 2008). Each cell layer within the epidermis is characterized by the specific expression of genes encoding for different keratins (Fuchs \& Green, 1980). Hence, keratin 14 (K14) together with keratin 5 (K5) are early expression markers of proliferative active keratinocytes of the stratum basale. As keratinocytes move towards the suprabasal layers, the expression of K5 (basic, type II) as well as K14 (acidic, type I) is down-regulated and expression of keratin 1 (K1) and keratin 10 (K10) is switched on, forming an even more stable framework of keratin filaments that is linked to intercellular desmosomes (Fuchs \& Green, 1980; Leigh et al, 1993). Therefore, K1 (basic, type II) and K10 (acidic, type I) are the first proteins to be synthesized when keratinocytes commit to terminal differentiation (reviewed by (Candi et al, 2005)) (see Figure 2). Under pathologic conditions the expression pattern of keratins is generally modified. For example, the expression of genes encoding for keratin 6 (K6) and keratin 16 (K16) is enhanced under hyperproliferative conditions (Stoler et al, 1988). In normal epidermis K6 and K16 are only expressed during wound healing (Lane et al, 1985; McGowan \& Coulombe, 1998).

Later during differentiation granular cells synthesize keratohyalin granules, which can in rodent epidermis be subdivided into L-and F-granules (Steven et al, 1990). F-granules are predominantly composed of profilaggrin, a histidine-rich precursor of filaggrin. During the late stages of terminal keratinocyte differentiation profilaggrin is released from F-granules and proteolytically processed into filaggrin monomers, which aggregate the keratin cytoskeleton, promoting the cell collapse into flattened, enucleated squames (Smith et al, 2006). It has been well established that the monomers are subsequently cleaved into free amino acids forming the natural moisturizing factor (NMF) of the stratum corneum (Brown \& McLean, 2012; Rawlings \& Harding, 2004). Filaggrin is the main keratin-aggregating protein in mammalian stratum corneum (reviewed by (Matsui \& Amagai, 2015)) and loss-of-function mutations in the gene encoding for filaggrin cause ichthyosis vulgaris, which is characterized clinically by dry skin, accompanied by scaling. Filaggrin mutations have been reported as strong predisposing factor for atopic dermatitis (Irvine et al, 2011; Thyssen et al, 2013). 
Although mouse models have often demonstrated remarkable similarity to human epidermal differentiation, there are a few discrepancies. F-granules, for example, have been described both in human as well as in rodent epidermis (Ishida-Yamamoto et al, 1993), whereas Lgranules are found exclusively in rodent epidermis (especially mouse). Its major constituent is loricrin, a glycine-serine-cysteine-rich protein, synthesized in the granular layer (Mehrel et al, 1990; Steven et al, 1990). In mouse epidermis loricrin accumulates into discrete L-granules (Yoneda et al, 1992), whereas in human epidermis it doesn't accumulate in any granular structure (Ishida-Yamamoto et al, 1993). Loricrin comprises up to 70-80\% of the total protein mass of the cornified envelope (CE) and therefore confers to its mechanical resistance (Steinert \& Marekov, 1995; Steinert \& Marekov, 1999).

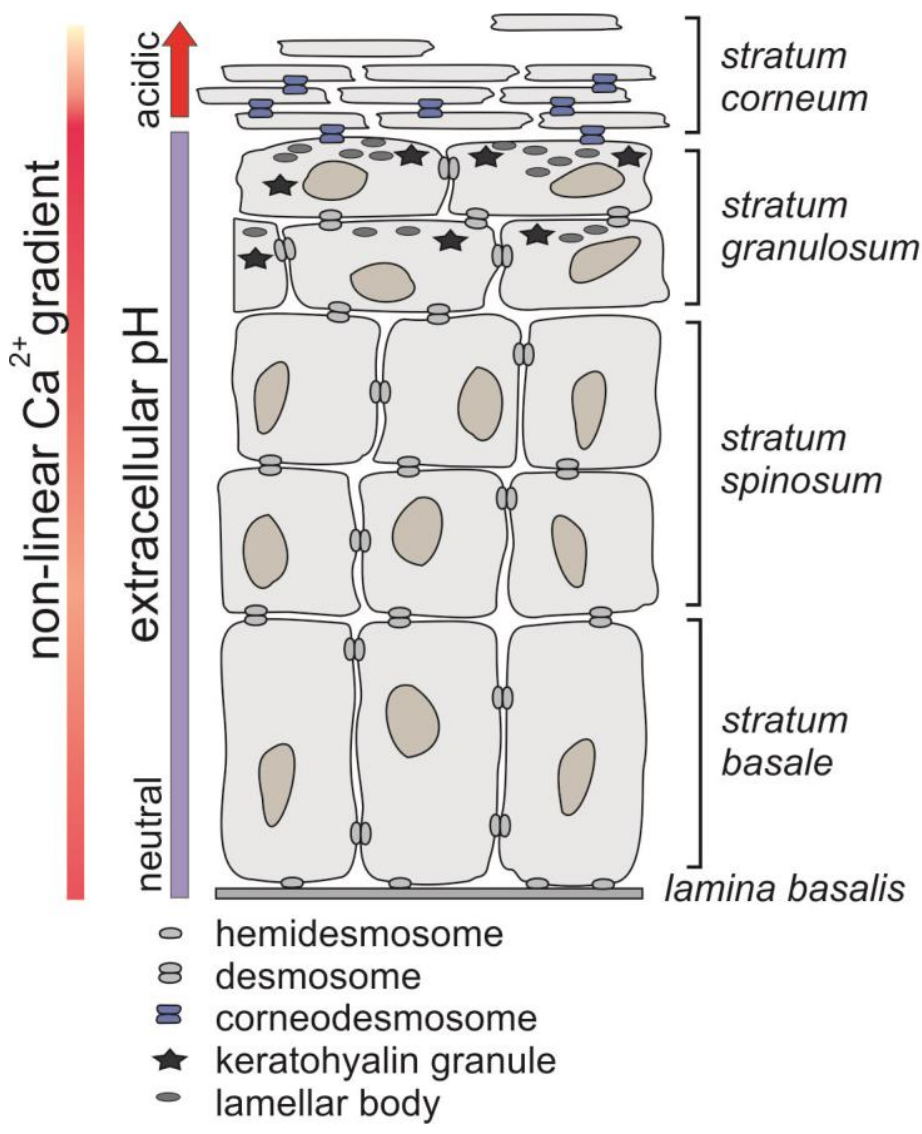

cornification
cornified envelope, filaggrin

late differentiation

loricrin and profilaggrin

early differentiation

keratins 1 and 10

proliferation

keratins 5 and 14

\section{Figure 2: Schematic illustration of the multi-layered epidermis.}

The stratum basale is attached to the basement membrane via hemidesmosomes, and consists of proliferating keratinocytes. During cornification, keratinocytes start migrating from the stratum basale, through transitional layers, and finally forming corneocytes. In the suprabasal layers the keratinocytes are interconnected by desmosomes. Lamellar bodies (LBs) as well as keratohyalin granules are found in the stratum granulosum. The non-linear calcium-gradient as well as the extracellular $\mathrm{pH}$ are depicted in the illustration. On the right, key molecular markers are shown. The illustration was kindly provided by Dr. Stina Schiller. 


\subsubsection{Formation of the cornified envelope (CE)}

As already mentioned, the epidermis serves as a general barrier, but the two most important barrier properties of the skin are creating a barrier against water loss and against the entry of microorganisms, respectively (Elias \& Choi, 2005). The CE is the functional key element of this barrier, which is composed of corneocytes embedded in lipid lamellae. For CE formation, several precursor proteins, including loricrin, filaggrin, involucrin and small proline-rich proteins are assembled directly underneath the plasma membrane of corneocytes. Concomitantly, the cell membrane disintegrates and the structural proteins are covalently crosslinked by transglutaminases, which are calcium-dependent enzymes (Eckert et al, 2005). In general, the CE functions to provide structural and mechanical integrity of terminally differentiated keratinocytes in the stratum corneum.

\subsubsection{The formation of lipid lamellae}

In parallel with the process of CE formation, the intercellular lipid envelope is arranged. Therefore, the extracellular space of corneocytes is filled with sheets of lipid lamellae. This arrangement of lipid lamellae is most important for the skin barrier to prevent epidermal water loss as well as in responding to environmental and mechanical assaults (reviewed by (Matsui \& Amagai, 2015)). The main components of the lipid envelope are ceramides, cholesterol, fatty acids and cholesterol esters (Wertz, 2000) and some of these lipids become covalently bound by ester bonds the CE proteins to form a lipid envelope surrounding the stratum corneum (reviewed by (Nishifuji \& Yoon, 2013)). The formation of the lipid lamellae is dependent on the maturation and secretion of lamellar bodies (LBs), a continuous tubularvesicular structure expanding from the trans-Golgi network (TGN) (Elias et al, 1998; Madison, 2003; Norlen, 2001) (see Chapter 1.2.4). During keratinocyte differentiation, precursor lipids, such as glucosylceramide (GlcCer), are incorporated into lamellar bodies (LBs) and subsequently secreted into the extracellular space between the stratum granulosum and the lower stratum corneum (reviewed by (Feingold, 2009)) (see Figure 3). Consequently, GlcCers are hydrolyzed to ceramides by $\beta$-glucocerebrosidase (Tybulewicz et al, 1992) and arranged into the intercellular space of the stratum corneum. This step is essential for the development of an epidermal lipid-barrier within the cornified layers (Elias et al, 2000; Nishifuji \& Yoon, 2013). 


\subsubsection{Lamellar bodies (LBs) and barrier homeostasis}

LBs are membrane bilayer encircled secretory organelles, occurring especially in keratinocytes of the upper spinous layers and in the stratum granulosum (reviewed by (Feingold, 2009)). Besides the above mentioned precursor lipids, LBs transfer and secret epidermal proteases (kallikreins and cathepsins), protease inhibitors (e.g. lympho-epithelial Kazal-type-related inhibitor) and structural proteins (e.g. corneodesmosin) from the Golgi apparatus to the intercellular spaces of the granular layer (see Figure 3) (Raymond et al, 2008). Moreover, anti-microbial peptides like CRAMP (cathelicidin-related anti-microbial peptide) are also present in LBs playing most probably a role in innate immune responses of the skin (Aberg et al, 2008; Braff et al, 2005). Inhibition of LB formation and maturation impairs barrier homeostasis (Feingold, 2009). Concordantly, disruption of the epidermal barrier results in a homeostatic repair response that rapidly restores barrier function (Taljebini et al, 1996). The initiation of the repair response involves, inter alia, changes in the concentrations of extracellular calcium, especially in the stratum granulosum (Menon et al, 1992).

Normally, mammalian epidermis is characterized by an intraepidermal non-linear calcium gradient (see Figure 2). The highest concentrations of extracellular calcium are found in the granular layers (Mauro et al, 1998), while calcium concentration in the stratum corneum is very low (Proksch et al, 2008). Under basal conditions, the high levels of calcium restrict LB secretion to low levels sufficient in maintaining barrier homeostasis. Following barrier perturbations, epidermal calcium concentrations decline and LB exocytosis is stimulated (Lee et al, 1992; Menon \& Elias, 1991; Menon et al, 1994). In addition to regulating LB secretion, high epidermal calcium concentrations also regulate the expression of differentiation markers as well as the formation of the CE (Elias et al, 2002; Yuspa et al, 1989). 


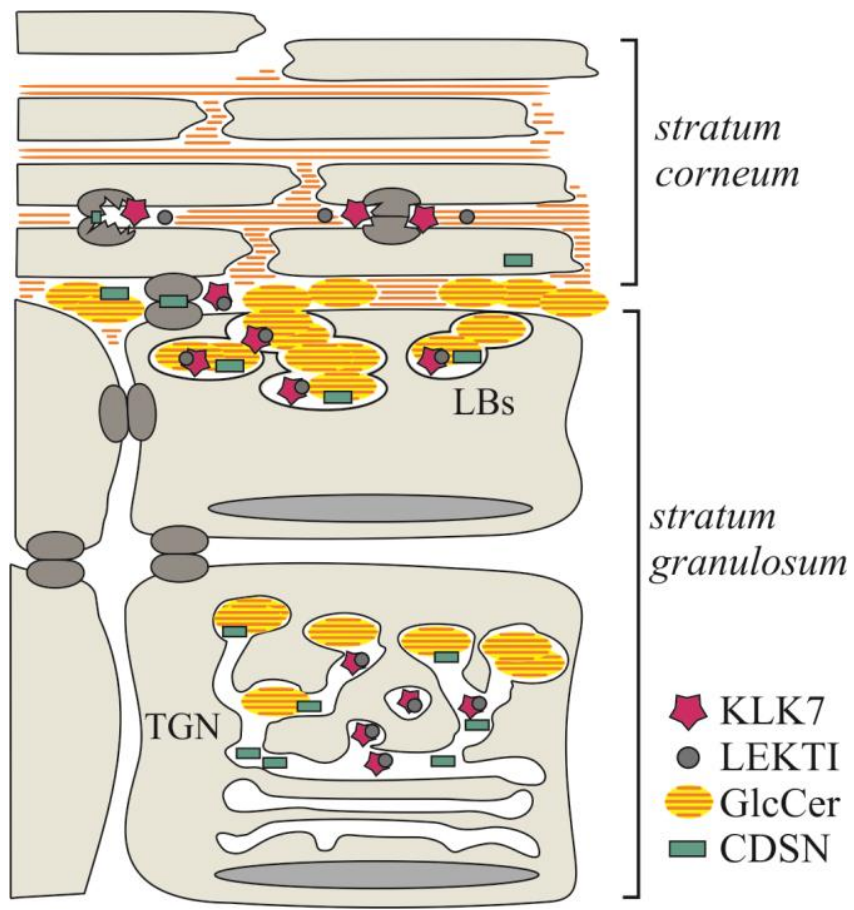

Figure 3: Schematic illustration of formation and maturation of LBs.

LBs are membrane bilayer-encircled secretory organelles, derived from the TGN. LBs are unique to mammalian epidermis and the maximal numbers are present in keratinocytes in the stratum granulosum. They deliver cargoes to the stratum granulosum/corneum interface, including the serine-protease KLK7 (red) and its inhibitor LEKTI (darkgrey), as well as glucosylceramides (GlcCer, yellow) and corneodesmosin (CDSN, blue), where they are secreted in order to form the CE. The LB-derived precursor lipids are subsequently modified and arranged to the lipid lamellae (yellow). The illustration was kindly provided by Dr. Stina Schiller.

\subsubsection{Desquamation, the shedding of corneocytes}

At the final steps of terminal differentiation, corneodesmosomes, which are modified desmosomal structures mediating cell-cell-adhesion between corneocytes, are dissolved by specialized proteases, and continuously shed off by a process called desquamation (reviewed by (Candi et al, 2005)). The major extracellular components of corneodesmosomes are desmoglein1, desmocollin 1, and corneodesmosin (CDSN), the latter being unique to corneodesmosomes and secreted by LBs (Ishida-Yamamoto \& Kishibe, 2011; Serre et al, 1991). The degradation process of corneodesmosomes is carefully controlled by a number of proteases, the most important are the kallikrein-related peptidases such as kallikrein 5 (KLK5) and kallikrein7 (KLK7) (Deraison et al, 2007) and their inhibitors, including the lymphoepithelial Kazal-type related inhibitor (LEKTI) (Caubet et al, 2004). 
The desquamation of corneocytes from the stratum corneum surface is orchestrated by changes in the $\mathrm{pH}$ (Elias et al, 2002; Hachem et al, 2003). The $\mathrm{pH}$ of neonatal both human and rodent stratum corneum is neutral, but an acidic mantle is gradually developed in the stratum corneum. The $\mathrm{pH}$ directly regulates epidermal permeability barrier homeostasis, and affects stratum corneum integrity, cohesion and desquamation (Hachem et al, 2003). Furthermore, the $\mathrm{pH}$ controls the activity of the serine-proteases KLK5 and KLK7 by regulating the interaction with their inhibitor LEKTI. A neutral $\mathrm{pH}$ allows strong interaction between kallikreins and LEKTI, however, as the $\mathrm{pH}$ acidifies along the stratum corneum, this complex dissociates, allowing the serine proteinases to cleave its corneodesmosomal targets (Deraison et al, 2007).

\subsubsection{Epidermal homeostasis and associated skin diseases}

Cell proliferation, differentiation and cell death occur sequentially and the epidermis regenerates continuously throughout the life of an organism. In humans the estimated epidermal turnover time is 40 -56 days (Gelfant, 1976; Halprin, 1972), whereas in mice it is 8 -10 days (Potten et al, 1987). The succession of proliferation, differentiation and desquamation is subjected to a well-orchestrated equilibrium named homeostasis (Blanpain \& Fuchs, 2009). Disturbances of this process, predominantly caused by mutations in genes encoding for epidermal barrier components, result in cutaneous diseases like ichthyoses. Ichthyoses refer to a large, clinically and etiologically heterogeneous group of cornification disorders, which are characterized by visible scaling and/or hyperkeratosis of the skin. Ichthyoses are caused by mutations in various different genes important for keratinocyte differentiation and epidermal barrier function, including genes encoding for CE components (e.g. filaggrin) and genes encoding for enzymes involved in lipid metabolism (e.g. glucocerebrosidase) (Akiyama, 2011b; Schmuth et al, 2013).

\subsection{CEDNIK syndrome}

In 2005, Sprecher et al. described for the first time a neurocutaneous disorder called CEDNIK (CErebral Dysgenesis, Neuropathy, Ichthyosis, and Keratoderma) syndrome (OMIM 609528) in seven individuals from two unrelated consanguineous Arab Muslim families from Northern Israel. CEDNIK syndrome is a rare autosomal recessive disorder, which is uniformly fatal in middle childhood (5 to 12 years). The clinical picture of all patients is characterized by 
microcephaly, facial dimorphism, severe neurological impairment, psychomotor retardation, palmoplantar keratoderma, and late-onset ichthyosis (see Figure 4). After a normal gestation, severe developmental failure of the nervous system and cutaneous symptoms with a progressive worsening appeared within the first months of life (between 5 and 11 months of age) (Sprecher et al, 2005).

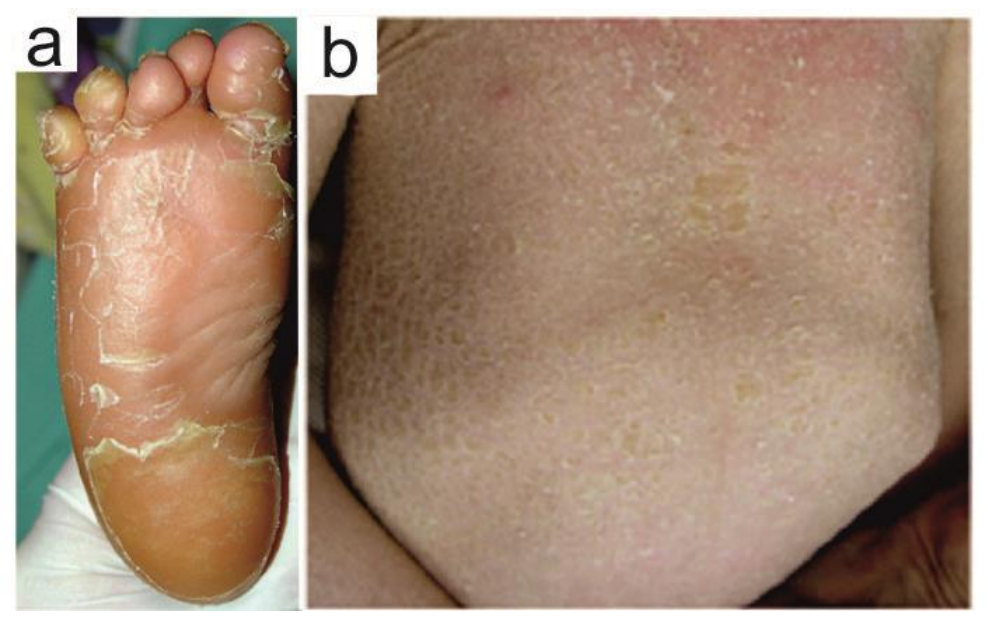

Figure 4: Cutaneous phenotype of CEDNIK syndrome patients (modified from Sprecher et al. 2005).

(a) CEDNIK syndrome patients suffer from a severe thickening of the plantar skin (keratoderma) and (b) lamellar ichthyosis, which is distributed over the whole abdominal surface of the body.

CEDNIK syndrome was found to result from loss-of-function mutations in the SNAP29 gene, encoding for the soluble n-ethylmaleimide sensitive factor attachment protein (SNAP) receptor (SNARE) 29 protein. Until today, seven mutations in the SNAP29 gene have been identified, some of which are frameshift mutations, leading to truncated proteins (Ben-Salem et al, 2015; Fuchs-Telem et al, 2011; McDonald-McGinn et al, 2013; Sprecher et al, 2005). Electron microscopy revealed that the epidermis of CEDNIK patients, aside from normalappearing LBs, exhibits numerous vesicular structures of varying size in the stratum granulosum and stratum corneum. These structures showed retained GlcCer, as well as KLK5 and KLK7, suggesting that SNAP29 deficiency results in markedly impaired maturation and secretion of LBs (Sprecher et al. 2005). The profound cutaneous pathology associated with CEDNIK syndrome unequivocally underscores the importance of SNAP29 during epidermal differentiation. In addition, CEDNIK syndrome is a part of a steadily growing group of cornification disorders associated with abnormal intracellular transport processes like ARC (Arthrogryposis-Renal dysfunction-Cholestasis) syndrome (Gissen et al, 2006) and MEDNIK (Mental retardation, Enteropathy, Deafness, Neuropathy, Ichthyosis, and Keratoderma) 
syndrome, which is caused by mutations in AP1S1 encoding a component of an adaptor protein complex involved in vesicle transport within or out of TGN (Montpetit et al, 2008).

\subsection{Membrane fusion and SNARE proteins}

During epidermal differentiation, membrane fusion between vesicles and target membranes is a crucial step. SNARE proteins have been recognized as the core machinery to catalyze membrane fusion in all the trafficking steps of the secretory pathway (reviewed by (Jahn \& Scheller, 2006)). Trafficking usually involves four central steps: vesicle budding, transport, tethering and fusion of the vesicle with the target membrane (reviewed by (Bonifacino \& Glick, 2004)).

In general, vesicle budding is mediated by protein coats, which can be subdivided into clathrin and non-clathrin coats like COPI and COPII (coat protein complex I/II). Clathrincoated vesicles transport their cargo from the plasma membrane to endosomes and from the TGN to endosomes (Stoorvogel et al, 1996). COPI primarily mediates traffic from the Golgi to the endoplasmic reticulum (ER), whereas COPII mediates the opposite direction (Barlowe et al, 1994; Letourneur et al, 1994; Waters et al, 1991). After budding, vesicles are transported to their acceptor membrane, followed by the initial interaction between a vesicle (e.g., lamellar body) and its target membrane (reviewed by (Cai et al, 2007)). This process is called tethering and it is most probably the earliest stage at which specificity of vesicular trafficking is conferred (reviewed by (Whyte \& Munro, 2002)). The final step of trafficking is the membrane fusion, which is mediated by SNAREs.

\subsubsection{SNAREs and their structure}

SNARE proteins comprise a superfamily of 24 members in Saccharomyces cerevisiae, 23 members in Caenorhabditis elegans, 54 members in Arabidopsis thaliana (Uemura et al, 2004), and at least 35 members in mammals (Bock et al, 2001). Although SNAREs vary markedly in size and structure, all SNARE proteins are characterized by an evolutionary conserved SNARE motif of 60-70 amino acids, containing heptad repeat sequences which are able to form coiled-coil structures (reviewed by (Chen \& Scheller, 2001)). The coiled-coil consists of a bundle of $\alpha$-helical peptides that are entwined around each other into a superhelical motif to form a stable structure (Apostolovic et al, 2010). A common structural 
principle of most SNAREs comprises a single transmembrane domain at the C-terminal end, which is connected to the SNARE motif by a short linker. Contrary to the conserved SNARE motifs, SNAREs contain different types of independently folded N-terminal domains, connected to the SNARE motif by a flexible linker, which is frequently palmitoylated (reviewed by (Jahn \& Scheller, 2006)) (see Figure 5).

\subsubsection{Classification of SNAREs}

SNAREs can be classified functionally into v-SNAREs, located on vesicular membranes, and t-SNAREs, located on target membranes (Sollner et al, 1993). In order to prevent ambiguity in the case of homotypic fusion events, SNAREs have been reclassified into Q- and RSNARES according to their highly conserved glutamine (Q) or arginine (R) residue (Fasshauer et al, 1998). A functional core complex consists of coiled-coils of four $\alpha$-helices, each contributed by another SNARE motif. Accordingly, the contributing SNARE motifs are classified into four subtypes: Qa, Qb, Qc- and R-SNAREs, based on their contribution in the central layer of the SNARE complex (see Figure 5). The ionic layer of the assembled core SNARE complex is composed of three glutamine (Q) residues, provided by Q-SNAREs, and one arginine $(\mathrm{R})$ residue, provided by a R-SNARE, respectively (Bock et al, 2001; Fasshauer et al, 1998). This heptad helical wheel is the only polar layer in the otherwise hydrophobic SNARE complex, and the residues are almost completely conserved within the SNARE family (Fasshauer et al, 1998; Ossig et al, 2000).

In general, three Q-SNAREs (Qabc-SNAREs) and one R-SNARE associate into a stable QabcR-SNARE complex, which catalyzes the fusion between the vesicle and target membrane (reviewed by (Ungar \& Hughson, 2003)) (see Figure 6). The coiled-coil structure can either be parallel (amino termini next to each other) or antiparallel, in which the amino terminus of one helix is close to the carboxyl terminus of the other (Lin \& Scheller, 1997). In vitro SNARE motifs assemble rather promiscuously in other, less stable conformations (Fasshauer et al, 1999), for example the anti-parallel Qaaaa (Misura et al, 2001b) or the parallel Qabab complex (Misura et al, 2001a). However, these complexes are lacking sufficient energy to drive complete membrane fusion. 


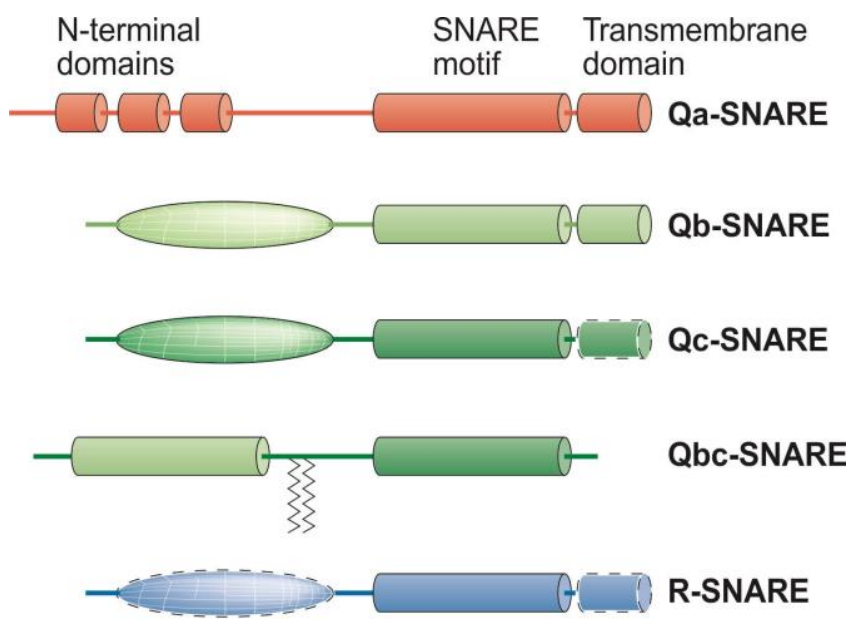

Figure 5: Schematic domain structure of SNAREs (modified from Jahn and Scheller 2006).

Qa-SNAREs have N-terminal antiparallel three-helix bundles (red), whereas the N-terminal domains of Qb-, Qc- and R-SNAREs are more diverse (oval shape). Qbc-SNAREs (e.g. SNAP29) represent a subfamily of SNAREs, which contain one Qb-SNARE motif and one Qc-SNARE motif, which are connected by a linker that is frequently palmitoylated (zig-zag lines in the figure). Qbc-SNAREs contain no transmembrane domain.

\subsubsection{SNARE core complex formation and dissasembly}

The key in understanding the fusion process of vesicles was the discovery of the assembly of the stable four helix bundle (QabcR). The association of the SNARE complex starts in transconfiguration at the N-terminal ends of the SNARE motifs and continues towards the Cterminal membrane anchors, pulling the membranes tightly together and forming the stable bundle of four parallel $\alpha$-helices. This 'zippering' of opposed membranes is associated with an extensive release of energy, sufficient to initiate membrane fusion ( $\mathrm{Li}$ et al, 2007; Wiederhold \& Fasshauer, 2009). The SNARE complex is extremely stable and resistant to SDS denaturation (Hayashi et al, 1994), protease digestion (Fasshauer et al, 1998; Hayashi et al, 1994; Poirier et al, 1998), and it is heat stable up to $90^{\circ} \mathrm{C}$ (Yang et al, 1999).

After fusion, the QabcR-SNARE complex resides in the low energy cis-configuration in the fused membrane (reviewed by (Jahn \& Scheller, 2006)). Subsequently, the disassembly of all SNARE complexes is mediated by the $\mathrm{AAA}^{+}$ATPase NSF factor (Littleton et al, 2001; Sollner et al, 1993). NSF by itself is not able to bind the surface of the SNARE complex and therefore the cofactor $\alpha$-SNAP (soluble NSF attachment protein) is required. Sufficient energy for the complete dissociation by the SNARE-SNAP-NSF complex is provided by several ATP-hydrolysis events, followed by the concomitant release of the individual SNAREs. 


\subsubsection{SNAREs and fusion specificity}

It was initially assumed that correct pairing of Qabc- and R-SNAREs mediates the specificity of membrane fusion; however, the extent to which SNAREs mediate fusion specificity remains elusive. As a consequence of the facts, that SNAREs form complexes rather promiscuously (see chapter 1.4.2) and that cognate SNAREs can be potentially substituted by non-cognate SNAREs of the same subclass, fusion specificity cannot be solely mediated by SNARE proteins (Fasshauer et al, 1999). Nowadays, it is a well established fact that together with Rabs (small GTPases of the Ras family), tethering factors play a critical role in confining the specificity of vesicle targeting (reviewed by (Cai et al, 2007)).

\subsection{SNAP29 and its interaction partners}

The notion that each trafficking event requires an individual set of SNAREs (Pelham, 2001) has led to the identification of many SNAREs within different families, for example the syntaxin and VAMP/synaptobrevin families. SNAP29 belongs to the family of SNAPs (soluble NSF attachment) proteins, which comprises four known members in mammals: SNAP23, SNAP25, SNAP29, and SNAP47 (Holt et al, 2006).

SNAP23, SNAP25 and SNAP47 contain two different SNARE motifs, whereas SNAP29 only contains one SNARE motif and a coiled-coil domain, comprising the second helix (see Figure 5) (Bock et al, 2001). Moreover, the members of the family of SNAP proteins differ from the previously described SNARE structure by lacking a transmembrane domain. In order to mediate membrane anchorage SNAP25 and SNAP23 contain post-translational palmitoylation in one or more cysteins found in the central part of these proteins, whereas SNAP29 and its closest homolog SNAP47 (Holt et al, 2006) are lacking any membrane-anchoring motif. In concordance with its structural features SNAP29 resides in the cytoplasm and associates with membranes transiently (Steegmaier et al, 1998; Wong et al, 1999). In contrast to other members of the family, SNAP29 has an amino acid stretch with a coiled-coil structure, being exclusive to this SNARE member, and a N-terminal asparigine-proline-phenylalanine (NPF) protein binding motif, that binds endocytic adaptors, such as EHD1 (Eps15 homology domain-containing protein 1). The EHD protein family has a role in endosomal membrane trafficking (Zhang et al, 2012), and it was shown, that EHD1 associates directly with SNAP29 through its NPF-motif (Rotem-Yehudar et al, 2001). 
As already mentioned, the SNARE complex for intracellular trafficking consists of one helix contributed by the R-SNARE and three by the Q-SNAREs (Bonifacino \& Glick, 2004) (see chapter 1.4.2). Despite the fact that most SNAREs contribute one helix to the trans-SNARE complex, members of the SNAP25 family contribute two helices. In this context, one helix originates from syntaxin and VAMP, respectively and two helices originate from the respective SNAP member (Bonifacino \& Glick, 2004) (see Figure 6).

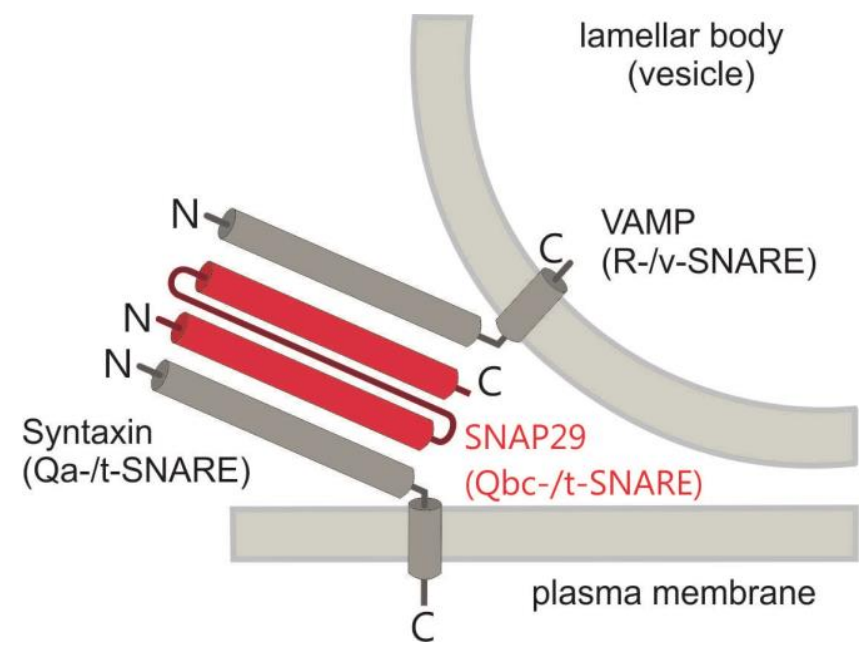

Figure 6: Schematic illustration of the assembly of the acceptor complex (Qabc-SNARE complex).

The cytosolic Qbc-SNARE SNAP29 (red) interacts with two membrane-bound SNAREs, a Qa-SNARE (Syntaxin) and an R-SNARE (VAMP). One helix is contributed by the RSNARE, another one by the Qa-SNARE and two by the Qbc-SNARE. The illustration was kindly provided by Dr. Stina Schiller.

\subsubsection{SNAP29 in membrane fusion events}

SNAP25 and SNAP23 are the most extensively studied members of the SNAP protein family; however SNAP29 has been studied much less. The SNAP29 gene comprises five exons (Rapaport et al, 2010) and it is ubiquitously expressed in a variety of tissues. Four of the seven mutations described so far in the SNAP29 gene are in immediate proximity or directly in the coiled-coil domain (see Chapter 1.3), indicating that mutations in this domain interfere with the efficiency of the interaction of SNAP29 with other SNARE proteins (McDonaldMcGinn et al, 2013).

Mammalian SNAP29 appears to participate in a wide range of fusion events and consistently, it is distributed on multiple internal membranes including Golgi, endosomes, and lysosomes, where it interacts with plasma membranes and with multiple intracellular Qa-SNAREs such 
as syntaxins (Steegmaier et al, 1998; Wong et al, 1999) (see Figure 6). SNAP29 is a general component involved in membrane trafficking (Hohenstein \& Roche, 2001), amongst others in endocytotic recycling and cell motility (Rapaport et al, 2010). SNAP29 has been proposed to promote, as well as inhibit, membrane fusion (Hohenstein \& Roche, 2001; Schardt et al, 2009; Steegmaier et al, 1998; Su et al, 2001; Wesolowski et al, 2012; Wong et al, 1999). It has been suggested that SNAP29 functions as a regulator of SNARE complex disassembly and that it additionally modulates the recycling of the SNARE components after the fusion process ( $\mathrm{Su}$ et al, 2001). More recently, it was demonstrated that Drosophila SNAP29 (dSNAP29) interacts with the late-Golgi dsyntaxin16, suggesting that dSNAP29 participates at fusion events in the Golgi or TGN (Xu et al, 2014). Furthermore, it was recently shown that a SNAP29 is required for the fusion of autophagosomes with lysosomes, in both human and fruit fly (Itakura et al, 2012; Takats et al, 2013) (see chapter 1.6.2). These studies highlight the importance of SNAP29 mediating fusion events in diverse biological processes in eukaryotes.

\subsection{Autophagy}

As mentioned above, SNAP29 is directly involved in autophagy through controlling the fusion of autophagosomal membrane with the lysosomal membrane (Itakura et al, 2012; Takats et al, 2013) (see Chapter 1.6.2). Autophagy is an intracellular degradation process conserved among eukaryotes in which cytoplasmic contents are degraded within the lysosome. The resulting macromolecular constituents are recycled to generate energy to maintain cell viability (reviewed by (Feng et al, 2014)). There are three different types of autophagy: macroautophagy, microautophagy and chaperone-mediated autophagy. Macroautophagy is considered to be the prevalent form and is best characterized at the molecular level (hereafter referred to as autophagy; other types of autophagy are not discussed here) (reviewed by (Lamb et al, 2013)). It was originally believed that autophagy is a nonselective process (Kominami et al, 1983; Kopitz et al, 1990), induced as a survival mechanism in response to cellular stress. Over the past several years, however, a consensus has emerged that autophagosomal membranes can selectively recognize specific organelles and proteins, performing a cellular quality control. The most noted inducer of autophagy is nutrient starvation, but it can also be initiated by other stress stimuli, such as pharmacological agents (e.g. rapamycin and bafilomycin A1) (Barth et al, 2010) or endoplasmic reticulum 
(ER) stress (Ogata et al, 2006). It is therefore useful to distinguish between basal and induced autophagy (Mizushima, 2005).

Autophagy is initiated by the formation of an isolation membrane, which sequesters a region of the cytoplasm, containing cytosolic proteins and organelles. Subsequently, this structure expands around the targeted material and is finally sealed, forming autophagosomes, which represent specialized double-membrane organelles. The formation of the pre-autophagosomal structures is mainly regulated by the function of autophagy-related (Atg) proteins, which operate in a strict hierarchical order (Itakura \& Mizushima, 2010). Upon induction of autophagy a cytosolic form of microtubule-associated protein light chain 3 (LC3-I) is conjugated to phosphatidylethanolamine (PE) converting it into LC3-PE (LC3-II), which is associated with both the inner and outer autophagosomal membranes (reviewed by (Mizushima, 2007)). Autophagosomes transport their cargo to the lysosomes for degradation, where the outer membrane of the autophagosome fuses with the lysosome, converting it into an autolysosome (see Figure 7). Subsequently, the cytoplasmic content together with the inner membrane of the autphagosome are degraded by lysosomal hydrolases. Likewise, LC3-II is degraded in the autolysosomal lumen.

The origin of the isolation membrane remains unclear. However, it has become increasingly evident that in mammals it is originating from ER membranes (reviewed by (Lamb et al, 2013)). In addition, the expansion of the nascent autophagosome requires several other cellular compartments, such as the Golgi (van der Vaart \& Reggiori, 2010), the plasma membrane (Ravikumar et al, 2010) and mitochondria (Hailey et al, 2010).

\subsubsection{Measurement of autophagic flux}

In order to monitor autophagic activity and to further distinguish between basal and induced autophagy, several assays have been established. One important aspect should be considered in this context: autophagic activity does not directly correspond to the accumulation of autophagosmomes. Therefore, monitoring the number of autophagosomes is insufficient to measure autophagic activity. An accumulation of autophagosomes might be due to an induction of autophagy (e.g. by nutrient starvation) or a block in downstream steps, respectively. Therefore, it is important to measure the "autophagic flux", which enables to distinguish between these two possibilities. The term describes the dynamic process of autophagosome synthesis, the delivery of autophagosome cargoes to the lysosome, and the subsequent proteolytical degradation of these components (Mizushima \& Levine, 2010). 
Autophagic flux can be monitored by the turnover of the autophagosomal marker LC3-II (see figure 7), under the treatment with exogenous reagents like Bafilomycin A1, which prevents autophagosome-lysosome fusion and acts by inhibiting the vacuolar $\mathrm{H}^{+}$ATPase (Yamamoto et al, 1998). As a result, autophagosomal structures accumulate (Klionsky et al, 2008; Rubinsztein et al, 2009). However, measurement of LC3 turnover is not sufficient in order to monitor autophagic flux. Levels of other autophagy substrates, including p62 (also known as SQSTM1) have to be used to determine autophagic flux. p62 is incorporated into autophagosomes through direct binding to LC3 and it is subsequently degraded by the lysosome (Bjorkoy et al, 2005). Therefore, inhibition of autophagy leads to the accumulation of p62, whereas decreased levels of p62 are observed when autophagy is induced.

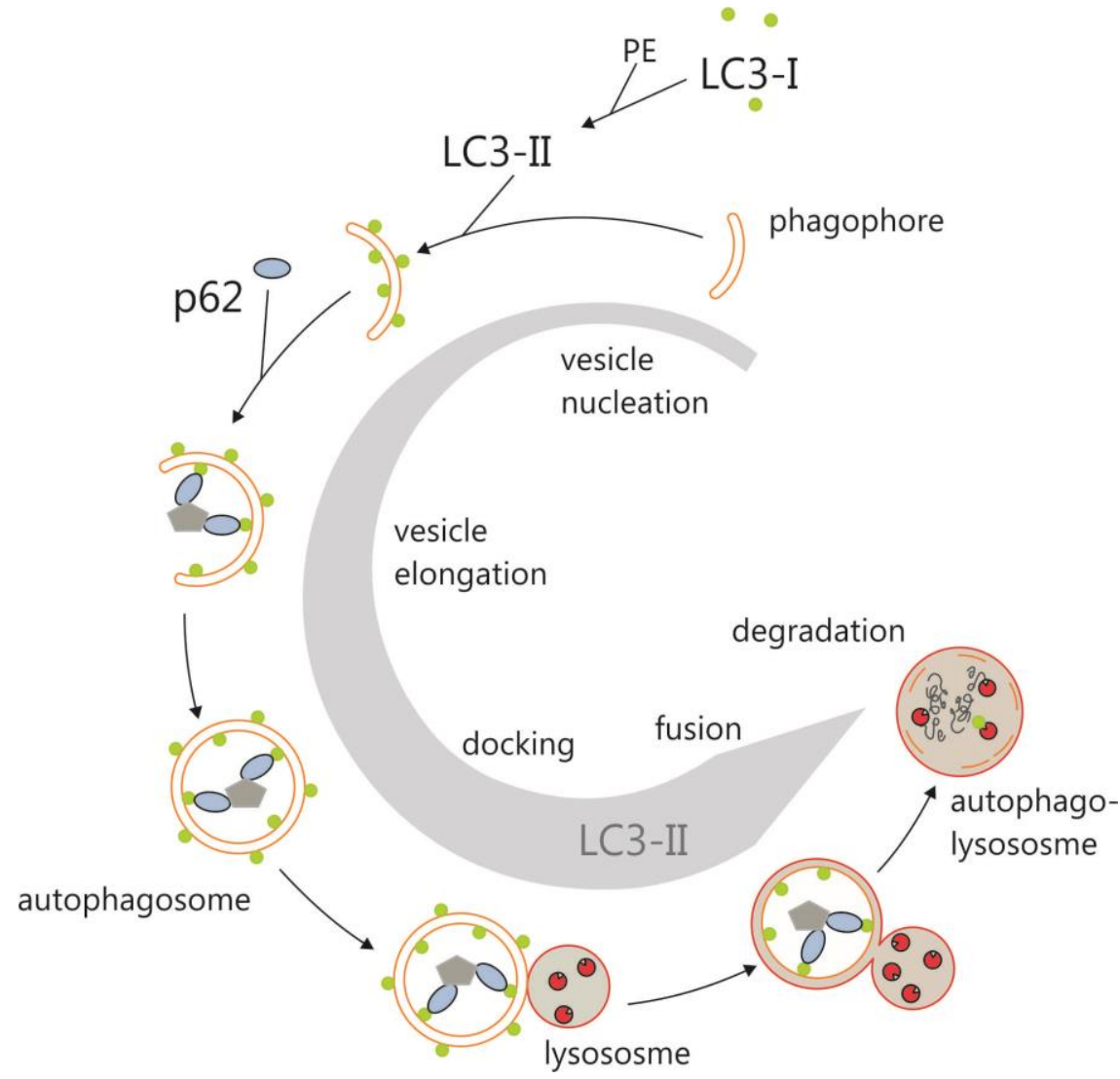

Figure 7: Incorporation of LC3-II and p62 into the autophagosome.

Cytosolic LC3-I is conjugated to PE and this step promotes integration of LC3-II into lipid membranes at the phagophore and autophagosomes. LC3B-II correlates with increased levels of autophagic vesicles. The ratio between LC3-I and LC3-II was used to determine changes in autophagosomal structures. The mammalian autophagy-specific substrate p62 interacts with poly-ubiquitinated protein aggregates and is incorporated into autophagosomes through direct binding to LC3-II. LC3-II as wells as p62 are subsequently degraded by the lysosome. Accumulation of p62 indicates a block in autophagic flux. The illustration was kindly provided by Dr. Stina Schiller. 


\subsubsection{Involvement of SNAP29 in autophagy}

Recently it has been shown in Drosophila melanogaster that Syntaxin17 (Stx17) is recruited to the outer membrane of autophagosomes to mediate fusion through its interactions with SNAP29 and VAMP7 (Takats et al, 2013). The fusion between autophagosome and lysosome is mediated by the three SNARE proteins: Stx17 (Qa-SNARE), SNAP29 (Qbc-SNARE), and the R-SNARE VAMP8 in human or VAMP7 in Drosophila melanogaster, respectively (see Figure 9) (Itakura et al, 2012; Takats et al, 2013). A knock-down of these genes in mammals resulted in accumulation of autophagosomes and a block of autolysosomal degradation during basal and starvation-induced autophagy. Furthermore, a role for Stx17 and its interaction partners in autophagy had also emerged in two other recent studies in mammals (Hamasaki et al, 2013; Itakura et al, 2012). Stx 17 was found to be located on autophagosomes and knockdown of Stx17 resulted in a blockage of autophagic flux. The formation of autophagosomes was not blocked, but knockdown of Stx17 blocked the fusion of autophagosmes with lysosomes. Therefore, Stx17 is required for the fusion of autophagosomes with lysosomes in mammalian cells (Itakura et al, 2012).

In addition, a recent study reported that SNAP29 activity and, correspondingly, autophagy are regulated by post-translational addition of $O$-linked $\beta$ - $N$-acetylglucosamine in Caenorhabditis elegans and HeLa cells (Guo et al, 2014). O-GlcNAcylation is a common post-translational modification that occurs on cytosolic as well as nuclear proteins (Mizushima, 2014). It has been established that the $O$-linked $\beta$ - $N$-acetylglucosamine $(O$-GlcNAc) transferase (OGT) modifies SNAP29 in a nutrient-dependent manner. This sugar modification of SNAP29 inhibits autophagosome-lysosome fusion as well as autophagic flux (Guo et al, 2014). Furthermore, it is reduced under starvation conditions, suggesting that signals under nutrientpoor conditions can activate the fusion of autophagosomes with lysosomes by blocking $O$ GlcNAcylation of SNAP29 (see Figure 8) (Mizushima, 2014). 
Introduction

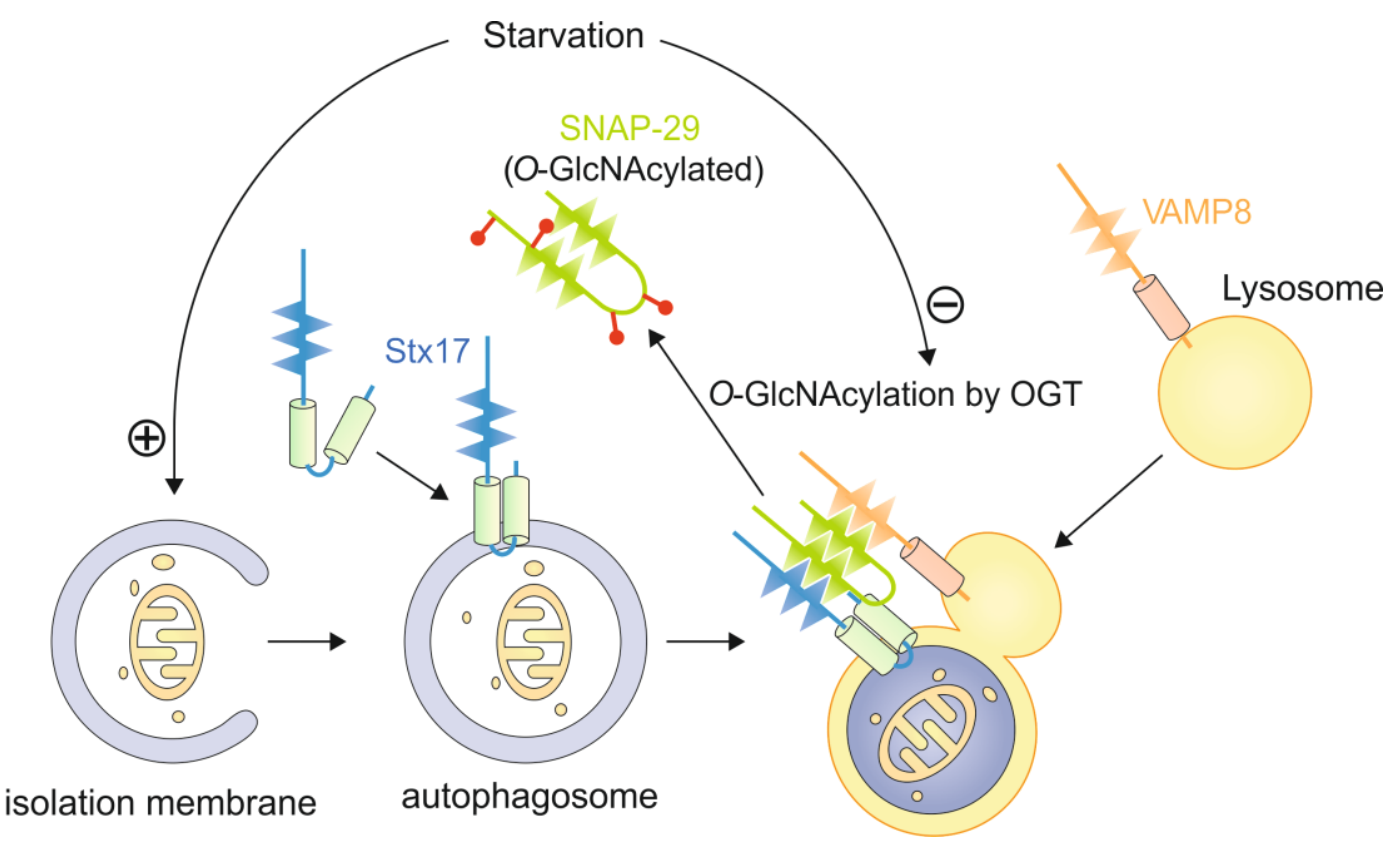

Figure 8: Regulation of autophagosome-lysosome fusion (modified from Mizushima 2014).

At the initiation step of autophagy the isolation membrane sequesters a part of the cytoplasm and eventually the autophagosome is formed. Afterwards, autophagosome-lysosome fusion proceeds in a process mediated by three SNARE proteins, Stx17 (blue), SNAP29 (green), and VAMP8 (orange). $O$-GlcNAcylation of SNAP29 (red symbols) by $O$-GlcNAc transferase (OGT) inhibits the formation of the SNARE complex, and thereby the fusion between the autophagosome and lysosome. 


\subsection{Aim of the study}

The profound cutaneous phenotypes associated with CEDNIK syndrome (Sprecher et al, 2005) unequivocally underscores the importance of SNAP29 during epidermal differentiation and in consideration of the very limited number of CEDNIK syndrome patients physiologically relevant models would greatly facilitate the study of the role of SNAP29 during epidermal differentiation.

Until now, merely a small number of genetic diseases associated with mutations in genes encoding for modulators of vesicle trafficking and mutations in genes encoding for SNAP molecules have been reported. CEDNIK syndrome is also part of a steadily growing group of cornification disorders associated with abnormal intracellular transport processes, including ARC syndrome and MEDNIK syndrome, as mentioned earlier.

To date, three non-mammalian Snap29-deficient models have been described. However, a mammalian model is still needed in order to further evaluate the role of SNAP29 in epidermal differentiation. Therefore, the aim of this study is to generate and characterize two different Snap29-deficient mouse models, in which mice are lacking SNAP29 ubiquitously or exclusively in keratinocytes. This approach is expected to dissect out the in vivo contribution of SNAP29 in normal epidermal barrier formation, to lead to an essential understanding of the complex biological function of SNAP29 in skin morphogenesis as well as barrier formation, and to mimic the cutaneous phenotype of the human CEDNIK syndrome.

Taken together, the Snap29-deficient mouse models will be characterized in this thesis regarding the following three guiding objectives:

- Generation of Snap29 knockout mouse lines and associated effects on epidermal differentiation and epidermal barrier function.

- Effects of Snap29 depletion on LB maturation and secretion in murine epidermis.

- Effects of Snap29 depletion on autophagic flux. 


\section{Materials}

\subsection{Biological material}

\subsubsection{Cell lines}

The used cell lines (murine primary fibroblasts, murine embryonic fibroblasts and murine primary keratinocytes) were isolated from the Snap $29^{-/-}$and Snap $29^{\mathrm{fl} / \mathrm{fl}} / \mathrm{K} 14-\mathrm{Cre}$ mouse line, respectively.

\subsection{Consumable supplies}

Table 1: Consumables

\begin{tabular}{|l|l|}
\hline Consumables & Manufacturer \\
\hline Blotting paper extra thick & Bio-Rad Laboratories, Munich GER \\
\hline Cell culture flasks $\left(25 \mathrm{~cm}^{3}, 75 \mathrm{~cm}^{3}, 175 \mathrm{~cm}^{3}\right)$ & Greiner Bio-One, Frickenhausen GER \\
\hline Cell strainer $(40 \mu \mathrm{m})$ & VWR, Darmstadt GER \\
\hline Cryo boxes & Nunc, Wiesbaden GER \\
\hline Cryo tubes $2 \mathrm{ml}$ & Greiner Bio-One, Frickenhausen GER \\
\hline Dispenser multipette ${ }^{\circledR}$ plus & Eppendorf, Hamburg GER \\
\hline Dispenser tips $(50 \mu 1,5 \mathrm{ml})$ & Eppendorf, Hamburg GER \\
\hline Falcon tubes $15 \mathrm{ml}, 50 \mathrm{ml}$ & Sarstedt, Nümbrecht GER \\
\hline Formvar-coated grids & Plano, Wetzlar GER \\
\hline Glass cover slips, round & Roth, Karlsruhe GER \\
\hline Kimtech wipes & Kimberly-Clarke Professional, Roswell USA \\
\hline Glass pipettes $(5 \mathrm{ml}, 10 \mathrm{ml}, 25 \mathrm{ml})$ & Brand, Wertheim GER \\
\hline Neubauer cell counting chamber & Brand, Wertheim GER \\
\hline Microscope slides & Thermo Scientific, Braunschweig GER \\
\hline Microscope slides SuperFrost ${ }^{\circledR}$ plus & Thermo Scientific, Braunschweig GER \\
\hline Parafilm ${ }^{\circledR}$ & Brand, Wertheim GER \\
\hline Pasteurpipettes $(230 \mathrm{~nm})$ & Brand, Wertheim GER \\
\hline PCR tubes & Sarstedt, Nümbrecht GER \\
\hline Petri dishes & Greiner Bio-One, Frickenhausen GER \\
\hline Pipettes $(10 \mu l, 100 \mu l, 200 \mu 1,1000 \mu l)$ & Eppendorf, Hamburg GER \\
\hline Pipette tips $(10 \mu l, 100 \mu l, 1000 \mu l)$ & Sarstedt, Nümbrecht GER \\
\hline
\end{tabular}




\begin{tabular}{|l|l|}
\hline PVDF membrane $(0.45 \mu \mathrm{M})$ & Roth, Karlsruhe GER \\
\hline Reaction tubes $(0,5 \mathrm{ml}, 1,5 \mathrm{ml}, 2,0 \mathrm{ml})$ & Eppendorf, Hamburg GER \\
\hline Scalpel, disposable & Feather, Osaka JPN \\
\hline Syringes, single use $(2 \mathrm{ml}, 5 \mathrm{ml}, 10 \mathrm{ml})$ & Braun, Melsungen GER \\
\hline Tissue culture multiwell plates $(24-w e l l)$ & Greiner Bio-One, Frickenhausen GER \\
\hline Tissue culture dish $(10 \mathrm{~cm})$ & Greiner Bio-One, Frickenhausen GER \\
\hline
\end{tabular}

\subsection{Equipment}

Table 2: Equipment

\begin{tabular}{|c|c|}
\hline Instrument & Manufacturer \\
\hline $2 \mathrm{k}$ side mounted CCD camera & Olympus, Hamburg GER \\
\hline 7900HT Fast Real-Time PCR System & Applied Biosystems, Foster City USA \\
\hline Analytical balance BP2100; MC1 & Sartorius, Goettingen GER \\
\hline Autoclave DE-65 & Systec, Wettenberg GER \\
\hline Benchtop centrifuge $5415 \mathrm{C}$ & Eppendorf, Hamburg GER \\
\hline $\mathrm{CO}_{2}$-Incubator & Sanyo, Munich GER \\
\hline Cold plate EG1150C & Leica Biosystems, Wetzlar GER \\
\hline Cryostat CM3050 S & Leica Biosystems, Wetzlar GER \\
\hline $\begin{array}{l}\text { Double Distilled Water System Arium® } \\
611 \mathrm{VF}\end{array}$ & Sartorius, Göttingen GER \\
\hline DU $640^{\circledR}$ Spectrophotometer & Beckmann, Munich GER \\
\hline EM 900 electron microscope & Carl Zeiss, Oberkochen GER \\
\hline FluoView 1000 confocal microscope & Olympus, Hamburg GER \\
\hline Freezer $-20,+4$ combination & Wielkart, GER \\
\hline Gel iX 20 Imager & Intas, Göttingen GER \\
\hline Hera freeze $-80^{\circ} \mathrm{C}$ freezer & Heraeus Instruments, Hanau GER \\
\hline Ice machine $\mathrm{ZBE} 30-10$ & Ziegra, Isernhagen GER \\
\hline Incubator model 200 & Memmer, Büchenbach GER \\
\hline LAS 4000 & Fujifilm, Düsseldorf GER \\
\hline Liquid Nitrogen container Apollo Biosafe & Messer Griesheim, Bad Soden GER \\
\hline Mega fuge 1,0, model G25 & Thermo Scientific, Braunschweig GER \\
\hline
\end{tabular}




\begin{tabular}{|l|l|}
\hline Microscope Axioskop 2 & Carl Zeiss, Oberkochen GER \\
\hline Microscope Axiovert 100 & Carl Zeiss, Oberkochen GER \\
\hline Microscope Axiovert Imager M1 & Carl Zeiss, Oberkochen GER \\
\hline Microscope BZ-9000 & Keyence, Neu-Isenburg GER \\
\hline Microtome RM2235 & Leica Biosystems, Wetzlar GER \\
\hline Microwave & Panasonic, Hamburg GER \\
\hline Mini-PROTEAN Tetra Cell & Bio-Rad Laboratories, Munich GER \\
\hline Mini Rocking Platform & Biometra, Goettingen GER \\
\hline NanoVuePlus ${ }^{\circledR}$ & GE Healthcare, Buckinghamshire UK \\
\hline Paraffin tissue floating bath & Medax, Neumünster GER \\
\hline pH meter & Schütt, Goettingen GER \\
\hline Pipetboy acu & Integra Biosciences, Fernwald GER \\
\hline Power Supply Ease 500 & Invitrogen, Karlsruhe GER \\
\hline Shaker & Infors, Bottmingen SWZ \\
\hline Steam oven FS20 & Braun, Kronberg/Taunus GER \\
\hline Sterile bench & Technoflow, Dubai UAE \\
\hline Thermomixer 5436 & Eppendorf, Hamburg GER \\
\hline T Gradient Thermo Block & Biometra, Goettingen GER \\
\hline TissueLyser LT & Qiagen, Hilden GER \\
\hline Trans-Blot ${ }^{\circledR}$ Turbo ${ }^{\text {TM }}$ Transfer System & Bio-Rad Laboratories, Munich GER \\
\hline Vortexer Vibrofix VF1 Electronic & IKA Labortechnik, Staufem GER \\
\hline Water bath & GFL, Großburgwedel GER \\
\hline
\end{tabular}

\subsection{Chemicals}

Table 3: Chemicals

\begin{tabular}{|l|l|}
\hline \multicolumn{1}{|c|}{ Chemical } & \multicolumn{1}{|c|}{ Manufacturer } \\
\hline $\begin{array}{l}\text { 5x HOT FIREpol }{ }^{(8)} \text { EvaGreen }^{(B)} \text { qPCR Mix } \\
\text { Plus (ROX) }\end{array}$ & Solis Biodyne, Tartu EST \\
\hline AEC substrate-chromogen & Agilent Technologies Inc., Santa Clara USA \\
\hline Agarose Saekem ${ }^{\circledR}$ & Fluka Chemie, Neu-Ulm GER \\
\hline Ammonium persulfate (APS) & Sigma-Aldrich, Taufkirchen GER \\
\hline
\end{tabular}




\begin{tabular}{|c|c|}
\hline Aqua ad iniectabilia & Braun, Melsungen GER \\
\hline BODIPY 493/503 & Thermo Scientific, Braunschweig GER \\
\hline Boric acid & Sigma-Aldrich, Taufkirchen GER \\
\hline Bradford Mix Roti ${ }^{\circledR}$ & Quant Roth, Karlsruhe GER \\
\hline Bromphenol blue & Sigma-Aldrich, Taufkirchen GER \\
\hline Calcium chloride $\left(\mathrm{CaCl}_{2}\right)$ & Merck, Darmstadt GER \\
\hline Target Retrieval Solution & Agilent Technologies Inc., Santa Clara USA \\
\hline Complete ULTRA Tablets Mini EDTA & Roche, Mannheim GER \\
\hline Dispase $^{(B)}$ II & Roche, Mannheim GER \\
\hline D-PBS & $\begin{array}{l}\text { GIBCO BRL, Life Technologies, Eggenstein } \\
\text { GER }\end{array}$ \\
\hline Dimethylsulfoxide (DMSO) & Sigma-Aldrich, Taufkirchen GER \\
\hline DNase & Qiagen, Hilden GER \\
\hline dNTP Mix (dATP, dCTP, dGTP, dTTP) & Thermo Scientific, Braunschweig GER \\
\hline EBSS & $\begin{array}{l}\text { GIBCO BRL, Life Technologies, Eggenstein } \\
\text { GER }\end{array}$ \\
\hline Eosin B & Merck, Darmstadt GER \\
\hline Ethanol absolute, $99,8 \%$ & Merck, Darmstadt GER \\
\hline Ethylenediaminetetraacetic acid (EDTA) & Sigma-Aldrich, Taufkirchen GER \\
\hline Faramount mounting medium, aqueous & Agilent Technologies Inc., Santa Clara USA \\
\hline Fluorescence mounting medium & Agilent Technologies Inc., Santa Clara USA \\
\hline Formaldehyde $4 \%$ & Serva, Heidelberg GER \\
\hline Gene Ruler (100bp \#SM0241) DNA ladder & Thermo Scientific, Braunschweig GER \\
\hline Glutaraldehyde $\mathrm{CH}_{2}\left(\mathrm{CH}_{2} \mathrm{CHO}\right)_{2}$, EM-grade & Science Services, Munich GER \\
\hline Glycine $\left(\mathrm{C}_{2} \mathrm{H}_{5} \mathrm{NO}_{2}\right)$ & Sigma-Aldrich, Taufkirchen GER \\
\hline Glycerol $\left(\mathrm{C}_{3} \mathrm{H}_{8} \mathrm{O}_{3}\right)$ & Merck, Darmstadt GER \\
\hline HBSS & $\begin{array}{l}\text { GIBCO BRL, Life Technologies, Eggenstein } \\
\text { GER }\end{array}$ \\
\hline HD Green & Intas, Göttingen GER \\
\hline Hematoxylin solution, Mayer's & Roth, Karlsruhe GER \\
\hline Hepes & PAA, Coelbe GER \\
\hline Hoechst33342 & Life Technologies, Eggenstein GER \\
\hline
\end{tabular}




\begin{tabular}{|c|c|}
\hline Isopropanol $\left(\mathrm{C}_{3} \mathrm{H}_{8} \mathrm{O}\right)$ & Merck, Darmstadt GER \\
\hline Magnesium Chloride $\left(\mathrm{MgCl}_{2}\right)$ & Merck, Darmstadt GER \\
\hline Marker VI, protein ladder & Applichem, Chicago Illinois USA \\
\hline Methanol $\left(\mathrm{CH}_{3} \mathrm{OH}\right)$ & Mallinckrodt Baker, Griesheim GER \\
\hline Nile Red & Sigma-Aldrich, Taufkirchen GER \\
\hline Nonidet P40 & Sigma-Aldrich, Taufkirchen GER \\
\hline Tissue-Tek ${ }^{\circledR}$ O.C.T. ${ }^{\text {TM }}$ compound & Sakura, Alphen aan den Rijn NL \\
\hline $\mathrm{OsO} 4(2 \%)$ & Science Services, Munich GER \\
\hline Paraformaldehyde (PFA) & Merck, Darmstadt GER \\
\hline PhosSTOP Phosphatase Inhibitor Cocktail & Roche, Mannheim GER \\
\hline Ponceau S & Sigma-Aldrich, Taufkirchen GER \\
\hline Potassium Chloride $(\mathrm{KCl})$ & Merck, Darmstadt GER \\
\hline Propylen oxide $\left(\mathrm{C}_{3} \mathrm{H}_{6} \mathrm{O}\right)$ & Sigma-Aldrich, Taufkirchen GER \\
\hline Roti ${ }^{\circledR}$-Free Stripping Buffer & Roth, Karlsruhe GER \\
\hline Sodium chloride $(\mathrm{NaCl})$ & Merck, Darmstadt GER \\
\hline Sodium dodecyl sulfate (SDS) & Roth, Karlsruhe GER \\
\hline Sodium hydroxide $(\mathrm{NaOH})$ & Merck, Darmstadt GER \\
\hline Trifluoroacetic acid (TFA) & Merck, Darmstadt GER \\
\hline Toluidine blue & Sigma-Aldrich, Taufkirchen GER \\
\hline Tris-Base & Merck, Darmstadt GER \\
\hline Triton $\mathrm{X}-100$ & Merck, Darmstadt GER \\
\hline Trypsin inhibitor soybean & $\begin{array}{l}\text { GIBCO BRL, Life Technologies Eggenstein } \\
\text { GER }\end{array}$ \\
\hline TSA blocking reagent & Perkin Elmer, Waltham USA \\
\hline $\begin{array}{l}\text { Vectashield Mounting Medium for } \\
\text { Fluorescence with DAPI 1:1200 }\end{array}$ & Vector Laboratories, Burlingham CA/USA \\
\hline$\beta$-mercaptoethanol $\left(\mathrm{C}_{2} \mathrm{H}_{6} \mathrm{OS}\right)$ & Merck, Darmstadt GER \\
\hline
\end{tabular}




\subsection{Buffers, solutions and media}

Frequently used buffers, solutions and media were prepared as listed below.

Table 4: Buffers, solutions and media

\begin{tabular}{|c|c|}
\hline & Receipt/ Manufacturer \\
\hline \multicolumn{2}{|l|}{ Cell culture } \\
\hline 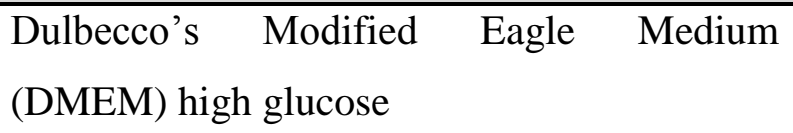 & PAA, Coelbe GER \\
\hline Freezing medium (fibroblasts) & $\begin{array}{l}40 \% \text { DMEM } \\
40 \%(v / v) \text { FBS } \\
20 \%(v / v) \text { DMSO }\end{array}$ \\
\hline Freezing medium (keratinocytes) & $\begin{array}{l}40 \% \text { keratinocyte growth medium } \\
40 \%(\mathrm{v} / \mathrm{v}) \mathrm{FBS} \\
20 \%(\mathrm{v} / \mathrm{v}) \mathrm{DMSO}\end{array}$ \\
\hline Fetal Bovine Serume (FBS) & Biochrom AG, Berlin GER \\
\hline Penicillin-Streptomycin (100x) & PAA, Coelbe GER \\
\hline Trypanblue solution $0,4 \%$ & Sigma-Aldrich, Taufkirchen GER \\
\hline Trypsin/EDTA & Biochrom AG, Berlin GER \\
\hline Keratinocyte growth medium & Provitro, Berlin GER \\
\hline Accutase & Biowest, Nuaillé FRA \\
\hline Gentamicin/Amphotericin B500X & $\begin{array}{l}\text { GIBCO BRL, Life Technologies Eggenstein } \\
\text { GER }\end{array}$ \\
\hline Dispase ${ }^{\circledR}$ II & $\begin{array}{l}\rightarrow \text { dissolved in Hepes-buffered saline } \\
(50 \mathrm{mM} \text { Hepes/KOH pH 7.4, } 150 \mathrm{mM} \mathrm{NaCl}) \\
(10 \mathrm{mg} / \mathrm{ml})\end{array}$ \\
\hline \multicolumn{2}{|l|}{ Gel electrophoresis } \\
\hline TBE & $\begin{array}{l}\text { 89mM Tris-Base } \\
\text { 89mM Boric acid } \\
\text { 2mM EDTA }\end{array}$ \\
\hline \multicolumn{2}{|l|}{ Protein biochemistry } \\
\hline Immunoblot transfer buffer, $\mathrm{pH} 8.3$ & $\begin{array}{l}0.192 \mathrm{M} \text { Glycine } \\
0.025 \mathrm{M} \text { Tris-Base } \\
20 \% \mathrm{MeOH}(\mathrm{v} / \mathrm{v})\end{array}$ \\
\hline
\end{tabular}




\begin{tabular}{|c|c|}
\hline & $0.01 \%$ SDS \\
\hline Ponceau S solution & $\begin{array}{l}0.2 \%(\mathrm{v} / \mathrm{v}) \text { Ponceau } \mathrm{S} \\
3 \%(\mathrm{v} / \mathrm{v}) \mathrm{TFA}\end{array}$ \\
\hline $4 \%$ paraformaldehyde (PFA) & $\begin{array}{l}\text { 3.6g PFA } \\
8 \mathrm{ml} \text { PBS } \\
8 \text { drops } 1 \mathrm{M} \mathrm{NaOH} \\
\rightarrow \text { dissolve stirring at } 60^{\circ} \mathrm{C} \\
\rightarrow \text { ad } 50 \mathrm{ml} \text { with } \mathrm{PBS}\end{array}$ \\
\hline SDS running buffer, $\mathrm{pH} 8.3$ & $\begin{array}{l}\text { 0.192M Glycine } \\
0.025 \mathrm{M} \text { Tris-Base } \\
0.1 \% \text { SDS }\end{array}$ \\
\hline SDS splitting buffer, $\mathrm{pH} 4,7$ & $\begin{array}{l}\text { 30mM Tris-Base } \\
3 \% \text { SDS } \\
15 \% \text { Glycerine } \\
0,04 \% \text { Brompehnol blue } \\
\text { before use add } 10 \% \beta \text {-mercaptoethanol }(\mathrm{v} / \mathrm{v})\end{array}$ \\
\hline Homogenization buffer, $\mathrm{pH}$ 7,0 & $\begin{array}{l}1 \% \text { Triton } \mathrm{X}-100 \\
150 \mathrm{mM} \text { Hepes } \\
1 \mathrm{mM} \text { EDTA } \\
\text { ad } 10 \mathrm{ml} \mathrm{H}_{2} \mathrm{O} \\
\text { ad protease and phosphatase inhibitors }\end{array}$ \\
\hline \multicolumn{2}{|c|}{ Additional commonly used buffers } \\
\hline 10x PBS, pH 7.2 & $\begin{array}{l}1.5 \mathrm{M} \mathrm{NaCl} \\
30 \mathrm{mM} \mathrm{KCl} \\
80 \mathrm{mM} \mathrm{Na}_{2} \mathrm{HPO}_{4} \times 2 \mathrm{H}_{2} \mathrm{O} \\
10 \mathrm{mM} \mathrm{KH}_{2} \mathrm{PO}_{4}\end{array}$ \\
\hline 6x DNA loading buffer & $\begin{array}{l}0.5 \text { M EDTA } \\
50 \% \text { (v/v) Glycerol } \\
0.01 \% \text { Brompehnol blue }\end{array}$ \\
\hline
\end{tabular}




\subsection{Oligonucleotides}

The following oligonucleotides were utilized in this work.

Table 5: Oligonucleotides

\begin{tabular}{|c|c|}
\hline Gene & Oligonucleotide \\
\hline \multicolumn{2}{|l|}{ Genotyping } \\
\hline P1 & 5'-GCAGCGTCTGCATTGGATAC-3' \\
\hline $\mathrm{P} 2$ & 5'-CTTGCTATCCACCTGCCTTAG-3' \\
\hline P3 & 5'-CACACCTCССССТGAACCTGAAAC-3' \\
\hline P4 & 5'-CCACATGTAACCACCAGCCCTC-3' \\
\hline P5 & 5'-GTTCTAGGACTCCTCGCTGCCG-3' \\
\hline EIIa-Cre-s & 5'-CCTGGAAAATGCTTCTGTCCG-3' \\
\hline EIIa-Cre & 5'-GTGAAACAGCATTGCTGTCACTT-3' \\
\hline K14-Cre-up & 5'-GATGAAAGCCAAGGGGAATG-3' \\
\hline K14-Cre-down & 5'-CATCACTCGTTGCATCGACC-3' \\
\hline \multicolumn{2}{|c|}{ Oligonucleotides for quantitative Real-time PCR purchased from Qiagen, Hilden GER } \\
\hline Gene & Order number \\
\hline Snap29 & QT00153853 \\
\hline \multicolumn{2}{|c|}{ Oligonucleotides for quantitative Real-time PCR } \\
\hline$\beta$-actin_fwd & 5'-AGCCATGTACGTAGCCATCCA-3' \\
\hline$\beta$-actin_rev & 5'-TCTCCGGAGTCCATCACAATG-3' \\
\hline
\end{tabular}

\subsection{Ready to use reaction systems}

The following ready to use reaction systems were utilized in this work.

Table 6: Reaction systems

\begin{tabular}{|l|l|}
\hline \multicolumn{1}{|c|}{ Description } & \multicolumn{1}{c|}{ Manufacturer } \\
\hline Gel extraction and PCR Clean Up & Machery + Nagel, Düren GER \\
\hline KAPA mouse genotyping Hot Start Kit & Peqlab, Erlangen GER \\
\hline KAPA Hot Taq DNA polymerase & Peqlab, Erlangen GER \\
\hline $\begin{array}{l}\text { Roti }^{\circledR} \text {-Quant Protein quantification assay } \\
\text { according to Bradford }\end{array}$ & Roth, Karlsruhe GER \\
\hline RNase free DNase Set & Qiagen, Hilden GER \\
\hline
\end{tabular}




\begin{tabular}{|l|l|}
\hline RNeasy Mini Kit & Qiagen, Hilden GER \\
\hline $\begin{array}{l}\text { RevertAid H Minus First Strand cDNA } \\
\text { synthesis Kit }\end{array}$ & Thermo Scientific, Braunschweig GER \\
\hline $\begin{array}{l}\text { WesternBreeze Chemilumonescent } \\
\text { Immunodetection Systems anti mouse/rabbit }\end{array}$ & Applied Biosystems, Foster City USA \\
\hline
\end{tabular}

\subsection{Antibodies}

Primary and secondary antibodies were diluted in TSA blocking reagent (see Table 3) and concentrations are listed below.

Table 7: Primary antibodies

\begin{tabular}{|c|c|c|c|c|}
\hline Antigen & Antibody & Host & Manufacturer & Dilution \\
\hline$\beta$-actin & $\begin{array}{l}\text { Anti-human } \beta \text {-actin } \\
\text { clone AC-74 }\end{array}$ & mouse & $\begin{array}{l}\text { Sigma-Aldrich, } \\
\text { Taufkirchen GER }\end{array}$ & $1: 2500$ \\
\hline caspase 3 & $\begin{array}{l}\text { anti-mouse caspase } 3 \\
\text { clone AF835 }\end{array}$ & rabbit & $\begin{array}{l}\text { R\&D Systems, } \\
\text { Minneapolis USA }\end{array}$ & $1: 1000$ \\
\hline CDSN & $\begin{array}{l}\text { anti-human } \\
\text { corneodesmosin clone } \\
\text { MBS713765 }\end{array}$ & rabbit & $\begin{array}{l}\text { Emelca Bioscience, } \\
\text { Breda NL }\end{array}$ & $1: 500$ \\
\hline $\begin{array}{l}\text { CHOP/ } \\
\text { GADD153 }\end{array}$ & $\begin{array}{l}\text { anti-mouse CHOP } \\
\text { clone NBP2-13172 }\end{array}$ & rabbit & $\begin{array}{l}\text { Novus Biologicals, } \\
\text { Littleton USA }\end{array}$ & $1: 250$ \\
\hline filaggrin & $\begin{array}{l}\text { anti-mouse filaggrin } \\
\text { clone PRB-417P }\end{array}$ & rabbit & Covance, Freiburg GER & $1: 10000$ \\
\hline GlcCer & $\begin{array}{l}\text { rabbit anti- } \\
\text { glucosylceramide }\end{array}$ & & $\begin{array}{l}\text { Glycobiotech }^{\text {TM }} \text {, Kükels } \\
\text { GER }\end{array}$ & $1: 500$ \\
\hline involucrin & $\begin{array}{l}\text { anti-mouse involucrin } \\
\text { clone ab28057 }\end{array}$ & rabbit & Abcam, Cambridge UK & $1: 4000$ \\
\hline keratin 6 & $\begin{array}{l}\text { anti-mouse keratin } 6 \\
\text { clone PRB-169P }\end{array}$ & rabbit & Covance, Freiburg GER & $1: 1500$ \\
\hline keratin 10 & $\begin{array}{l}\text { anti-mouse keratin } 10 \\
\text { clone ab9026 }\end{array}$ & mouse & Abcam, Cambridge UK & $1: 100$ \\
\hline keratin 14 & anti- mouse keratin 14 & rabbit & Covance, Freiburg GER & $1: 1000$ \\
\hline
\end{tabular}




\begin{tabular}{|c|c|c|c|c|}
\hline & clone PRB-155P & & & \\
\hline Ki67 & $\begin{array}{l}\text { anti-mouse Ki67 clone } \\
556003\end{array}$ & mouse & $\begin{array}{l}\text { BD Biosciences, } \\
\text { Heidelberg GER }\end{array}$ & $1: 100$ \\
\hline KLK7 & $\begin{array}{l}\text { anti-mouse kallikrein } \\
7 \text { clone } \mathrm{C}-15\end{array}$ & goat & $\begin{array}{l}\text { Santa Cruz } \\
\text { Biotechnology, } \\
\text { Heidelberg GER }\end{array}$ & $1: 500$ \\
\hline $\mathrm{LC} 3 \mathrm{~B}$ & $\begin{array}{l}\text { anti-mouse LC3B } \\
\text { clone \#2275 }\end{array}$ & rabbit & $\begin{array}{l}\text { Cell Signaling } \\
\text { Technologies }^{\circledR} \text {, } \\
\text { Cambridge UK }\end{array}$ & $1: 1000$ \\
\hline p62 & $\begin{array}{l}\text { anti-mouse p62 clone } \\
\# 5114\end{array}$ & rabbit & $\begin{array}{l}\text { Cell Signaling } \\
\text { Technologies }^{\circledR}, \\
\text { Cambridge UK }\end{array}$ & $1: 1000$ \\
\hline SNAP29 & $\begin{array}{l}\text { anti-mouse SNAP29 } \\
\text { clone } 111303\end{array}$ & rabbit & $\begin{array}{l}\text { Synaptic Systems, } \\
\text { Göttingen GER }\end{array}$ & $1: 300$ \\
\hline
\end{tabular}

Table 8: Secondary antibodies

\begin{tabular}{|l|l|l|l|}
\hline \multicolumn{1}{|c|}{ Antibody } & \multicolumn{1}{|c|}{ Host } & \multicolumn{1}{c|}{ Manufacturer } & Dilution \\
\hline $\begin{array}{l}\text { Alexa-Fluor } \\
\text { rabbit } 488 \text { goat anti- }^{\circledR}\end{array}$ & goat & Dianova, Hamburg GER & $1: 800$ \\
\hline $\begin{array}{l}\text { Alexa-Fluor } \\
\text { mouse } 594 \text { goat anti- }\end{array}$ & goat & Dianova, Hamburg GER & $1: 800$ \\
\hline biotinylated anti-rabbit IgG & goat & $\begin{array}{l}\text { Vector Laboratories, } \\
\text { Burlingame USA }\end{array}$ & $1: 300$ \\
\hline biotinylated anti-mouse IgG & goat & $\begin{array}{l}\text { Vector Laboratories, } \\
\text { Burlingame USA }\end{array}$ & $1: 300$ \\
\hline biotinylated anti-goat IgG & rabbit & $\begin{array}{l}\text { Vector Laboratories, } \\
\text { Burlingame USA }\end{array}$ & $1: 300$ \\
\hline $\begin{array}{l}\text { Streptavidin, Peroxidase } \\
\text { conjugate }\end{array}$ & Merck, Darmstadt GER & $1: 500$ \\
\hline
\end{tabular}




\subsection{Software and online tools}

The following software and online tools were used during this project:

Table 9: List of utilized software and online tools

\begin{tabular}{|l|l|l|}
\hline Label & Supplier/web address & application \\
\hline $\begin{array}{l}\text { CorelDraw Graphics } \\
\text { Suits X5 }\end{array}$ & Corel Corporation, Ottawa CA & Image Editing \\
\hline E!ensembl & http://www.ensembl.org & Genome browser \\
\hline Endnote X1.01 & Thomson Reuters, New York USA & Literature management \\
\hline ImageJ & $\begin{array}{l}\text { National Institutes of Health, } \\
\text { Bethesda USA }\end{array}$ & $\begin{array}{l}\text { Western blot } \\
\text { quantification by } \\
\text { densiometry }\end{array}$ \\
\hline LAS4000 & Fujifilm, Düsseldorf GER & $\begin{array}{l}\text { Imaging System, Western } \\
\text { Blot }\end{array}$ \\
\hline Microsoft Office & Microsoft, Unterschleißheim GER & Word, Powerpoint, Excel \\
\hline Pubmed & http://www.ncbi.nlm.nih.gov/pubmed & Literature request \\
\hline PyRAT & $\begin{array}{l}\text { Scionics Computer Innovation } \\
\text { GmbH }\end{array}$ & $\begin{array}{l}\text { Animal Facility } \\
\text { Management }\end{array}$ \\
\hline TierBase & MPI Freiburg & animal breeding \\
\hline
\end{tabular}




\section{Methods}

\subsection{Animals}

The use of mice as an animal model was approved by the local authorities for Animal Care and Use of the state of Lower Saxony (LAVES, Oldenburg, Germany). All experiments were performed in accordance with animal policies of the University Department of Medicine Göttingen and the Max Planck Institute for Experimental Medicine. B6N;B6N-Snap29 tmla(EUCOMM)Wtsi heterozygous knock-in mice were derived from a mouse line generated by the Wellcome Trust Sanger Institute (WTSI) Mouse genetics programme (MGP) and were provided by the European Mutant Mouse Archive (EMMA: www.emmanet.org) (strain number, EM:04440). All animals were housed under standard conditions in the animal facilities of the University Department of Medicine Göttingen and the Max Planck Institute for Experimental Medicine in Göttingen at $20-22^{\circ} \mathrm{C}$ and $50-55 \%$ relative humidity, on a 12 hour light and 12-hour dark schedule.

\subsection{Genotyping mice}

\subsubsection{Isolation of genomic DNA}

Genomic DNA was extracted from tail biopsies using the KAPA Mouse Genotyping Kit according to manufacturer's specification. Sequences of primers (see Table 5), the expected product sizes and the corresponding genotypes are given in Figure 11.

\subsection{Microbiology}

\subsubsection{Sterilization and autoclavation}

Liquids as well as S1-waste were autoclaved at $120^{\circ} \mathrm{C}$ and $3 \mathrm{bar}$ for $20 \mathrm{~min}$. Plastic pipette tips were autoclaved at $120^{\circ} \mathrm{C}$ and 3 bar and subsequently dried at $70^{\circ} \mathrm{C}$ for 4 hours. Glass pipettes used for cell culture were washed in a dish washer at $40^{\circ} \mathrm{C}$. Afterwards, glass pipettes were sealed with cotton wool and sterilized at $190^{\circ} \mathrm{C}$ for at least 4 hours. 


\subsection{Molecular methods}

\subsubsection{Isolation of nucleic acids}

\subsubsection{Extraction of nucleic acids from agarose gels}

Extraction of specific DNA fragments from 1\% agarose gels (see Chapter 3.4.6.1) was performed using the NucleoSpin ${ }^{\circledR}$ Gel extraction and PCR clean up Kit by Macherey-Nagel according to the instructions of the manufacturer. Prior to extraction, DNA fragments were separated by agarose gel electrophoresis and subsequntly, the fragment of interest was excised using a clean scalpel.

\subsubsection{RNA isolation from primary fibroblasts}

Total RNA was isolated from primary murine fibroblasts using the RNeasy Mini Kit by Qiagen. Cells were harvested as described in chapter 3.4.7.2 and disrupted using RLT buffer. Depending on the number of pelleted cells, either $350 \mu 1$ or $600 \mu l$ RLT buffer was added to the pellet or further steps were carried out according to the manufacturer's specifications. In order to remove any residual contaminating DNA, DNAse treatment was performed. Finally, RNA quantification and its assessment of purity were determined by spectrophotometry using the NanoVuePlus ${ }^{\circledR}$ (see Chapter 3.4.2) and the RNA was stored at $-80^{\circ} \mathrm{C}$ until further use.

\subsubsection{Enzymatic manipulation of nucleic acids}

\subsubsection{Complementary DNA (cDNA) synthesis}

cDNA was synthesized using the Revert Aid H Minus First Strand cDNA Synthesis Kit according to the manufacturer's instructions. This kit contains an Oligo (dT) $)_{18}$ primer, which hybridizes to the poly(A)-tail of the messenger RNA (mRNA). The RevertAid H Minus Reverse Transcriptase lacks a ribonuclease $\mathrm{H}$ activity and therefore, it does not degrade RNA in RNA-DNA hybrids during synthesis of the first strand cDNA.

In order to synthesize cDNA, $1 \mu \mathrm{g}$ of total RNA was mixed with $1 \mu$ l Oligo $\mathrm{d}(\mathrm{T})$ primer, auqa bidest was added to $12 \mu \mathrm{l}$ and incubated at $70^{\circ} \mathrm{C}$ for $5 \mathrm{~min}$. In the mean time, a premix consisting of buffer, RNase inhibitor and dNTPs was prepared. Subsequently, the premix was added to the reaction mix and incubated at $37^{\circ} \mathrm{C}$ for $5 \mathrm{~min}$. Thereafter, $1 \mu 1$ reverse transcriptase was added and the cDNA was synthesized at $42^{\circ} \mathrm{C}$ for $60 \mathrm{~min}$. The reaction was 
stopped by incubation at $70^{\circ} \mathrm{C}$ for $10 \mathrm{~min}$ and the synthesized cDNA was stored at $-20^{\circ} \mathrm{C}$ until further use.

\subsubsection{Polymerase Chain Reaction (PCR)}

The PCR was first described in 1986 (Mullis et al, 1986) and it is commonly used to synthesize multiple copies of a segment of DNA. PCR is very precise and can be used to amplify a specific DNA target from a mixture of DNA molecules. It consits of three steps which are repeated several times (cycles, $n$ ): strand separation of the target DNA by heat (denaturation), binding of the oligonucleotide primers to the single stranded target sequence (annealing) and the extension of the annealed primers by a thermostable DNA polymerase (polymerization). Primer sequences are listed in Table 5. Primers P1, P2, and P3 were used for genotyping Snap29-1- mice and the size of the respective PCR products are depicted in Figure 11. Primers P4, P5, and P2 were used for genotyping Snap29 $9^{\mathrm{fl} / \mathrm{fl}} / \mathrm{K} 14-\mathrm{Cre}$ mice. Primers EIIa-Cre-s and EIIa-Cre were used for genotyping EIIa-Cre mutant mice.

Standard PCR reactions were carried out in a total volume of $20 \mu l$ using $1 \mu$ l of template and a Hot Taq DNA polymerase.

Reaction mix:

- $\quad 1 \mu 1$ template

- $\quad 0,5 \mu 1$ forward primer

- $\quad 0,5 \mu 1$ reverse primer

- $\quad 4 \mu 1$ enhancer solution

- $2 \mu 1$ S-buffer

- $\quad 1 \mu 1$ Hot Taq DNA polymerase (diluted 1:10)

- $\quad 1 \mu 1 \mathrm{dNTPs}$

- $\mathrm{H}_{2} \mathrm{O}$ ad $20 \mu \mathrm{l}$

PCR conditions:

- $95^{\circ} \mathrm{C}, 3 \mathrm{~min}$ initial denaturation

- $\quad 95^{\circ} \mathrm{C}, 30 \mathrm{sec}$ denaturation

- $55^{\circ} \mathrm{C}, 30 \mathrm{sec}$ primer annealing

- $\quad 72^{\circ} \mathrm{C}, 30$ sec elongation

- $\quad 72^{\circ} \mathrm{C}, 10 \mathrm{~min}$ final extension 
Primers K14-Cre-up and K14-Cre-down were used for genotyping K14-Cre mutant mice. PCR conditions were:

- $\quad 95^{\circ} \mathrm{C}, 2 \mathrm{~min}$ initial denaturation

- $\quad 95^{\circ} \mathrm{C}, 30 \mathrm{sec}$ denaturation

- $\quad 60^{\circ} \mathrm{C}, 45 \mathrm{sec}$ primer annealing

- $\quad 72^{\circ} \mathrm{C}, 45 \mathrm{sec}$ elongation

- $\quad 72^{\circ} \mathrm{C}, 5 \mathrm{~min}$ final extension

PCR reactions were analyzed on $1 \%$ agarose gels containing HD Green (5 $\mu 1$ HD Green per $100 \mathrm{ml}$ agarose). Fragments were visualized under ultraviolet light (UV) using the gel documentation system by Intas (see Chapter 3.4.6.1).

\subsubsection{Quantitative Real-time PCR}

For quantitative Real-time PCR, total RNA was extracted from primary murine fibroblasts using the RNeasy Mini Kit according to manufacturer's instructions. Afterwards, cDNA was synthesized from $1 \mu \mathrm{g}$ of total RNA using the RevertAid H Minus First Strand cDNA synthesis Kit (see chapter 3.4.2). Analysis of Snap29 gene expression was carried out using EVA Green-based real-time PCR detection. For each reaction, 50ng of cDNA was used in a final reaction mixture of $10 \mu 1$, containing 1 pmol oligonucleotides and $2 \mu 1$ EvaGreen ${ }^{\circledR}$ qPCR Mix Plus. A passive reference dye (ROX) is included in this mix to normalize the fluorescent reporter signal. Each sample was analyzed in duplicate. Snap29 expression levels were normalized against $\beta$-Actin housekeeping gene. Quantitative Real-time PCR analyses were performed using the Applied Biosystems 7900HT Fast Real-Time PCR System. The sequences of the PCR primer pairs are shown in Table 5.

The PCR protocol was:

- $\quad 95^{\circ} \mathrm{C}, 15 \mathrm{~min}$ initial denaturation

- $\quad 95^{\circ} \mathrm{C}, 15 \mathrm{sec}$ denaturation

- $60^{\circ} \mathrm{C}, 20$ sec primer annealing

- $\quad 72^{\circ} \mathrm{C}, 20 \mathrm{sec}$ elongation

- and a final dissociation curve 


\subsubsection{Analysis of DNA}

\subsubsection{Agarose gel electrophoresis (AGE)}

DNA fragments generated by PCR (see Chapter 3.4.4) were subjected to agarose gel electrophoresis for analysis or preparative purposes. The phosphate backbone of the DNA molecule is negatively charged, therefore in an electric field, DNA fragments will migrate to the positively charged anode. DNA fragments exhibit different mobility due to the DNA fragment size and the pore size of the agarose gel. Accordingly, smaller fragments move faster than bigger ones, and consequently they can be separated by size.

Agarose gels were prepared by microwave heating a suitable amount of agarose in $1 \mathrm{x}$ TBE buffer, which also served as running buffer. The concentration of agarose in a gel was depended on the sizes of the DNA fragments to be separated. For visualization, a nucleic acid stain (HD Green ${ }^{\circledR}$ Safe DNA Dye) was added $(5 \mu 1$ per $100 \mathrm{ml}$ agarose). Samples were mixed with loading buffer (see Table 4) and electrophoresis was run at a current of $90 \mathrm{~V}$ for $45 \mathrm{~min}$. Fragment sizes were compared to an appropriate molecular weight size standard. Visualization of the DNA fragments was performed under UV light using a gel documentation system from Intas.

\subsubsection{Cell Biology}

\subsubsection{Preparation of primary murine fibroblasts}

Primary murine fibroblasts were obtained either from skin biopsies of neonatal or from embryonic mice. Mouse embryonic fibroblasts (MEFs) were isolated from mouse embryos collected at E19 or E20. The pregnant mouse was sacrificed by cervical dislocation, the uterine horns were dissected out and immersed in PBS containing 5\% P/S (v/v) for 1 hour at $4^{\circ} \mathrm{C}$. Tail biopsies for genotyping (see chapter 3.2) were taken and stored at $-20^{\circ} \mathrm{C}$. The following steps were carried out in a tissue culture hood using sterile instruments. The uterine horns were placed in a Petri dish and each embryo was separated from its embryonic sac and afterwards the head was dissected.

Neonatal mice were sacrificed by decapitation and rinsed in PBS containing 5\% P/S (v/v) for 1 hour at $4^{\circ} \mathrm{C}$. The mice were placed in a clean Petri dish containing PBS and 5\% P/S (v/v). The skin biopsies were collected using a razor blade and small pieces were placed in a $75 \mathrm{~cm}^{2}$ cell culture flask. The samples were allowed to dry for 20-30min. Subsequently, 10ml DMEM supplemented with $10 \%$ FBS (v/v) and $1 \%$ P/S (v/v), was carefully added to the culture flask 
in order to prevent detachment of the skin biopsies from the cell culture flask.

\subsubsection{Culture of primary murine fibroblasts}

Murine fibroblasts were cultured with DMEM (high glucose) supplemented with $10 \%$ FBS (v/v) and $1 \% \mathrm{P} / \mathrm{S}(\mathrm{v} / \mathrm{v})$ in a humified atmosphere at $37^{\circ} \mathrm{C}$ and $5 \% \mathrm{CO}_{2}$ until they reached confluency. Prior to passaging, the adherent cells were initially rinsed with $10 \mathrm{ml}$ PBS to remove any residual FBS as well as dead cells. Dissociation from the culture flask was achieved using $4 \mathrm{ml}$ trypsin/EDTA at $37^{\circ} \mathrm{C}$ for $5 \mathrm{~min}$. Trypsin was inactivated by addition of $10 \mathrm{ml}$ culture medium (complete), followed by centrifugation at $1000 \mathrm{rpm}$ for $5 \mathrm{~min}$. The supernatant was discarded and the pellet was resuspended in an adequate volume of medium. Depending on the cell density and growing behavior, cells were usually split at a ratio of 1:2 for primary fibroblasts and transferred into a new culture flask.

\subsubsection{Isolation of primary murine keratinocytes}

Newborn mice were sacrificed by decapitation and washed in D-PBS with $20 \mu \mathrm{g} / \mathrm{ml}$ Gentamicin/Amphotericin for at least 1 hour. Tail and limbs of the mice were cut off (for genotyping see Chapter 3.2) and the skin of was peeled off, most suitable in one piece. In order to detach the epidermis from the dermis, the skin was incubated for 16-18 hours in a dispase solution ( $1 \mathrm{ml}$ dispase, $5 \mathrm{ml} \mathrm{HBSS}, 4,8 \mu \mathrm{l} \mathrm{Gentamicin/Amphotericin)} \mathrm{at} 4{ }^{\circ} \mathrm{C}$ in a Petri dish. The following day, the epidermis was gently separated from the dermis with a pair of curved forceps. The epidermis was transferred into a new petri dish containing $2 \mathrm{ml}$ trypsinEDTA and incubated for $10 \mathrm{~min}$ at $37^{\circ} \mathrm{C}$. Afterwards, the epidermis was minced using a sclapel in order to separate single cells from the cell sheet and $4 \mathrm{ml}$ of trypsin-inhibitor were added to stop the reaction. The cell suspension was given trough a cell strainer $(40 \mu \mathrm{m})$ and subsequently centrifuged for $10 \mathrm{~min}$ at $500 \mathrm{rpm}$. The supernatant was discareded and the cell pellet was resuspended in an adequate volume of keratinocyte growth medium. Cells were seeded in culture flasks and the folowing day, the medium was changed to remove unattached cells.

\subsubsection{Cultivation of primary murine keratinocytes}

The cultivation of primary murine keratinocytes has been exceedingly difficult due to the frequent contamination with e.g. fibroblasts (Prose et al, 1967). In order to prevent contaminations with other cell types, primary keratinocytes were cultured in a serum-free 
medium with low calcium concentrations, which was supplemented with a mixture of several growth factors (see Table 4).

Additionaly, primary murine keratinocytes are highly responsive to calcium concentrations (Hennings et al, 1980). Higher calcium concentration than $0,09 \mathrm{mM}$ enhance terminal differentiation and therefore decrease proliferation of undifferentiated keratinocytes (Hennings et al, 1980; Roop et al, 1983). Concordantly, keratinocytes were cultured with special keratinocyte growth medium supplemented with Gentamicin/Amphotericin B $(1 \mathrm{ml}$ in $500 \mathrm{ml}$ medium) in a humified atmosphere at $37^{\circ} \mathrm{C}$ and $5 \% \mathrm{CO}_{2}$ until they reached confluency. The culture medium was changed every three to four days. In order to detach the cells from the culture surface, Accutase ${ }^{\circledR}$ was used, which is a mixture of proteolytic and collagenolytic enzymes isolated from crustaceans. The cells were washed with D-PBS to remove any residual medium and an adequate volume of Accutase $^{\circledR}$ was added, followed by 20-30min incubation at $37^{\circ} \mathrm{C}$. Accutase ${ }^{\circledR}$ leaves most surface proteins intact and it does not require a separate reagent to stop the reaction. After detachment of the cells, Accutase ${ }^{\circledR}$ was diluted with medium, followed by centrifugation at 500rpm for $10 \mathrm{~min}$. The supernatant was discarded, the pellet was resuspended in an adequate volume of medium and transferred into a new culture flask.

\subsubsection{Cell counting}

In order to determine the number of cells/ml, adherent cells were detached with trypsin/EDTA and resuspended in fresh culture medium (complete) as described before (see chapter 3.4.7.2

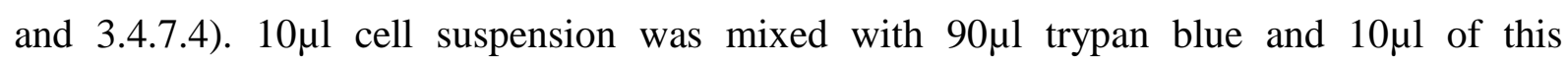
suspension was applied to a Neubauer counting chamber. Four large squares (each $1 \mathrm{~mm}^{2}$ ) were counted under a microscope and the number of cells was the sum of all the counted cells in all squares counted. The cell number per $\mathrm{ml}$ is: the sum of all counted cells $\mathrm{x} 2500 \mathrm{x}$ the dilution factor 10 .

\subsubsection{Freezing and thawing cells}

For cryo-preservation of fibroblasts, cells were sedimented at $1000 \mathrm{rpm}$ for $5 \mathrm{~min}$, the supernatant was discarded and the pellet was resuspended in $500 \mu l$ culture medium. Briefly, $500 \mu 1$ freezing medium was added and the suspension was transferred to cryo tubes. A cell freezing container filled with isopropanol was used to ensure a controlled cooling rate ($1^{\circ} \mathrm{C} / \mathrm{min}$ ) at $-80^{\circ} \mathrm{C}$. The following day, the cryo tubes were stored in liquid nitrogen for 
permanent storage at $-196^{\circ} \mathrm{C}$. For cryo-preservation of keratinocytes, cells were detached using Accutase ${ }^{\circledR}$ as described in Chapter 3.4.7.4 and centrifuged at 500rpm for 10min.

In order to cultivate frozen cells, cells were thawed under running tap water and transferred to $10 \mathrm{ml}$ culture medium (complete) and centrifuged at $1000 \mathrm{rpm}$ for $5 \mathrm{~min}$. The supernatant was discarded and the pellet was resuspended in an appropriate volume of culture medium and reseeded in a culture flask. The culture medium was renewed the following day to remove any residual DMSO and dead cells.

\subsubsection{Histopathological and immunohistochemical analysis}

\subsubsection{Tissue preparation, paraffin and OCT embedding, sectioning}

For assessment of the skin biopsies by immunohistochemistry, mice were sacrificed by decapitation directly after birth and dorsal skin grafts of newborn litters from knock-out and control groups were fixed overnight in $4 \%$ phosphate-buffered formaldehyde (PFA) and then automatically embedded in paraffin. Skin biopsies were dehydrated in an ascending enthanol series, incubated in xylene and afterwards in paraffin at $60^{\circ} \mathrm{C}$. Finally, the samples were embedded in paraffin and the blocks were stored at room temperature.

Prior to sectioning, the blocks were cooled down for at least 30min on a cooling plate. Afterwards, the blocks were sectioned with a rotary microtome in $3 \mu \mathrm{m}$ thick slices and placed on Superfrost ${ }^{\mathrm{TM}}$ Plus microscope slides. Sections were dried overnight at $42^{\circ} \mathrm{C}$ and stored at room temperature for several weeks.

For frozen sections, skin biopsies were embedded in O.C.T. ${ }^{\mathrm{TM}}$ (optimal cutting temperature compound) and stored at $-80^{\circ} \mathrm{C}$ until needed. Slices were sectioned with a cryostat at 5 to $10 \mu \mathrm{m}$, placed on Superfrost ${ }^{\mathrm{TM}}$ Plus microscope slides and air dried for 2 hours. If not needed the same day, cryo-sections were stored at $-80^{\circ} \mathrm{C}$ for several weeks.

\subsubsection{Haematoxylin and eosin staining (H\&E staining)}

H\&E staining can be used amongst others to identify morphologic changes in the epidermis. Hematoxylin stains nucleic acids in a blue-purple manner and eosin stains proteins nonspecifically (Cardiff et al, 2014).

Sections were deparaffinized by incubation in xylene for 10min (two times) and rehydrated with a descending ethanol series $(2 \mathrm{x} 100 \%, 96 \%, 75 \%, 50 \%$, and 30\%) for 5min each. Slides 
were incubated in $\mathrm{dH} 2 \mathrm{O}$ (5min), stained with Mayer's haematoxylin solution for $1 \mathrm{~min}$ and rinsed under running tap water for $10 \mathrm{~min}$. Subsequently, sections were counterstained with eosin staining solution for $5 \mathrm{~min}$. Sections were washed in $\mathrm{dH}_{2} \mathrm{O}$ and mounted with Faramount mounting medium. Pictures were taken with an Axioskop 2.

\subsubsection{Immunohistochemistry}

Protein levels were examined by staining of epidermal sections with the corresponding specific antibodies (Table 7). All immunohistochemical experiments were performed using age-matched littermates. $3 \mu \mathrm{m}$ paraffin-embedded sections of neonatal dorsal skin grafts were deparaffinized by incubation in xylene for 10min, rehydrated in a descendening ethanol series (2x $100 \%, 96 \%, 75 \%, 50 \%$, and 30\%) for $5 \mathrm{~min}$ each and incubated in $\mathrm{dH}_{2} \mathrm{O}$ for $5 \mathrm{~min}$. The sections were subjected to antigen retrieval in pre-heated target retrieval solutiom ( $\mathrm{pH}$ 6.1) in a steam oven for $25 \mathrm{~min}$. Afterwards, the sections were allowed to cool down to room temperature and washed three times with PBS. Endogenous peroxidase activity was quenched for $15 \mathrm{~min}$ at room temperature with $3 \% \mathrm{H}_{2} \mathrm{O}_{2}$ in PBS and washed three times with PBS. Prior to incubation with the primary antibody, the sections were incubated for at least $30 \mathrm{~min}$ at room temperature in TSA blocking reagent and then incubated with the respective primary antibodies overnight at $4{ }^{\circ} \mathrm{C}$ (see Table 7). Every incubation step was performed in a wet chamber preventing the slides from drying out. After every following step, slides were washed three times with PBS. The next day, sections were incubated with a biotinylated secondary antibody for $60 \mathrm{~min}$ at room temperature, followed by incubation with a HRPconjugated streptavidin for 40min at RT. Subsequently, the antibodies were visualized using AEC substrate-chromogen. Staining intensity was controlled under a microscope and the reaction was stopped with $\mathrm{dH}_{2} \mathrm{O}$. The slides were counterstained with hematoxylin for $1 \mathrm{~min}$ and rinsed under running tap water for $10 \mathrm{~min}$. Sections were washed in $\mathrm{dH}_{2} \mathrm{O}$ and mounted with Faramount mounting medium. Pictures were taken with an Axioskop 2. Sections labeled with only the secondary antibody served as controls.

\subsubsection{Immunofluorescence analysis}

Indirect immunofluorescence uses an unlabeled primary antibody (see Table 7), which recognizes the target antigen and binds to it. Afterwards, a secondary antibody conjugated to a fluorescent dye is used to bind the first antibody (see Table 8) (Odell \& Cook, 2013). 
Paraffin-embedded tissue sections were used, deparaffinized, antigen unmasked, and blocked with TSA blocking reagent as described in Chapter 3.4.8.3. Cryo-sections were fixed with ice cold methanol for $10 \mathrm{~min}$ at $-20^{\circ} \mathrm{C}$, washed with PBS and also blocked with TSA blocking reagent. The sections were incubated with the respective primary antibody overnight at $4^{\circ} \mathrm{C}$ in a wet chamber. The following day, the sections were washed extensively with PBS and incubated with the corresponding fluorescent-labeled secondary antibody, diluted 1:800 in blocking buffer, for $45 \mathrm{~min}$ at room temperature. Sections were washed three times with PBS and mounted with Vectashield mounting medium containing DAPI. Pictures were taken with an Axioskop 100 using the corresponding filter set to the fluorescent dye of the secondary antibodies. The pictures were automatically merged by the Axiovision software program.

In order to perform immunofluorescence analysis with cells, primary murine keratinocytes were seeded the previous day on glass cover slips in 24-well plates (5.000 cells per well, see Chapter 3.4.7.5). The following day, the cells were washed with PBS and fixed with 4\% PFA in PBS for 15min. Immunofluorescence was performed as described above.

\subsubsection{Nile red staining}

Nile red (9-diethylamino-5H-benzo[alpha]phenoxazine-5-one) is a lipophilic dye commonly used for the detection of lipids via fluorescence microscopy. The emission shifts from red to yellow according to the degree of hydrophobicity of lipids. Neutral lipids are stained in yellow, whereas polar lipids are stained in red (Diaz et al, 2008).

A stock solution of Nile red $\left(500 \mu \mathrm{g} / \mathrm{ml}\right.$ in acetone) was prepared, stored at $4{ }^{\circ} \mathrm{C}$, and protected from light. Shortly afterwards, a fresh Nile red staining solution $(2,5 \mu \mathrm{g} / \mathrm{ml})$ was prepared by adding $5 \mu \mathrm{l}$ of stock solution to $995 \mu \mathrm{l}$ of $70 \%$ glycerol and for nuclear staining $10 \mu \mathrm{l}$ DAPI $(1 \mu \mathrm{g} / \mathrm{ml})$ were added. A few drops of staining solution were applied to OCT-embedded frozen sections $(5 \mu \mathrm{m})$ and immediately mounted with fluorescence mounting medium. Nile red fluorescence was captured with an Axioskop 100. Fluorescence emission was detected in the range of $450-500 \mathrm{~nm}$.

\subsubsection{BODIPY 493/503 staining}

BODIPY 493/503 is another lipophilic fluorescent dye, which stains as well as Nile red neutral lipids and has been extensively used for lipid droplet labeling (Ohsaki et al, 2010). BODIPY 493/503 was dissolved in DMSO to give a stock solution of $1 \mathrm{mg} / \mathrm{ml}$ and stored at $20^{\circ} \mathrm{C}$. A fresh staining solution of BODIPY 493/503 diluted in PBS $(1 \mu \mathrm{g} / \mathrm{ml})$ was used for 
staining. $5 \mu \mathrm{m}$ frozen sections were fixed for $20 \mathrm{~min}$ with $4 \%$ PFA, washed with PBS and stained for $20 \mathrm{~min}$ at room temperature and mounted with fluorescence mounting medium. Images were taken with a FluoView 1000 confocal microscope (Olympus) at 1024x1024 pixel resolution using a 40x 0.90 NA UPLSAPO objective, 2-fold line averaging and a pinhole size corresponding to 1 Airy disc. BODIPY 493/503 was excited using the 488nm line of the Argon laser. Fluorescence emission was detected in the range of 500-600nm.

\subsubsection{Skin permeability assays}

In order to test potential epidermal barrier defects, inward as well as outward epidermal barrier function were analyzed. The fluid loss across the epidermis through evaporation was assessed by measuring the body weight of the mice (List et al, 2002). Therefore, neonatal mice were separated from their mothers to prevent dietary intake and kept at $37^{\circ} \mathrm{C}$. The body weight was measured every $15 \mathrm{~min}$ using a precision scale for approximately 3 hours. The rate of water loss was calculated by the reduction of body weight as a function of time.

Skin permeability was also assessed by dye diffusion using toluidine blue, which is an acidophilic metachromatic dye. It has an affinity for nucleic acids, and therefore stains tissues with a high DNA and RNA content (Sridharan \& Shankar, 2012). Sacrificed neonatal mice were rinsed in PBS and dehydrated in an ascending methanol series (25\%, 50\%, and 75\% methanol/PBS) followed by incubation with $100 \%$ methanol for $1 \mathrm{~min}$. Subsequently, the mice were rehydrated with the same series of methanol solutions (1min each), rinsed in PBS, and stained for $30 \mathrm{~min}$ in $0.1 \%$ toluidine blue/PBS. After destaining in PBS for $15 \mathrm{~min}$, the mice were photographed immediately.

\subsubsection{Electron microscopy}

Electron microscopy was performed at the Max Planck Institute of Experimental Medicine in the Department of Neurogenetics. Skin biopsies of sacrificed mice were immersed in fixation solution, consisting of $2.5 \%$ glutaraldehyde (EM-grade), and $4 \%$ formaldehyde in phosphate buffer (pH 7.3), according to Karlsson and Schultz (Karlsson \& Schultz, 1965) and suitably modified (Mobius et al, 2010). After postfixation with 2\% OsO4 and dehydration with ethanol, isopropanol, and propylene oxide, samples were embedded in Epon. Ultrathin sections were placed on formvar-coated grids and imaged with an EM 900 electron microscope with a $2 \mathrm{k}$ side mounted CCD camera. 


\subsection{Biochemical methods}

\subsubsection{Preparation of whole protein lysates}

For western blotting, whole protein lysates were either prepared from skin sections or from primary fibroblasts. Skin sections were disrupted and homogenized with lysis buffer (see Table 4) containing a protease-inhibitor mix using the TissueLyser LT for $45 \mathrm{~min}$ at $4{ }^{\circ} \mathrm{C}$. Extracts were put on ice and briefly sedimented at $13000 \mathrm{rpm}$ for $20 \mathrm{~min}$ at $4^{\circ} \mathrm{C}$. Supernatants were re-centrifuged for $15 \mathrm{~min}$.

In order to prepare protein lysates from cells, fibroblasts were harvested as described in Chapter 3.4.7.2. Cell lysis was carried out by resuspending the cell pellet in lysis buffer (see Table 4), followed by incubation for $30 \mathrm{~min}$ on ice. The cell lysates were sedimented to remove the residual cell debris $\left(15 \mathrm{~min}, 13000 \mathrm{rpm}\right.$, at $\left.4^{\circ} \mathrm{C}\right)$ and the supernatant was transferred into a new reaction tube.

\subsubsection{Bradford protein quantification}

Protein concentration was examined by Bradford assay (Bradford, 1976) using a ready to use Bradford solution according to the manufacturer's instructions. Protein concentration was photometrically measured $\left(\mathrm{OD}_{595}\right)$ and quantification was determined using a BSA calibration curve (serial dilutions, $0-10 \mu \mathrm{g} / \mathrm{ml}$ ).

\subsubsection{Polyacrylamide gel electrophoresis (SDS-PAGE)}

Proteins were separated according to their size using the polyacrylamide gel electrophoresis (SDS-PAGE). Therefore, protein lysates were mixed with 3\% SDS splitting buffer, containing B-mercaptoethanol, and heated for $10 \mathrm{~min}$ at $95^{\circ} \mathrm{C}$. SDS as a detergent, along with the reducing agent (to break down protein-protein disulphide bonds), disrupts the tertiary structure and coats the protein with an overall negative charge. The SDS-treated proteins have similar charge-to-mass ratios in an electric field; hence they can be separated according to their molecular weight. Separation of proteins via gel electrophoresis was performed using SDS-PAGE gels with a concentration of $12.5 \%$ or $15 \%$ for the separation gel and $5 \%$ for the stacking gel. Gels were loaded with $25-50 \mu \mathrm{g}$ whole protein lysates. 


\subsubsection{Western Blotting}

Western Blot technique allows protein visualization of one specific protein within a protein mixture by antibody detection. For semi-dry western blotting, the proteins were transferred onto a polyvinylidene difluoride (PVDF) membrane after horizontal SDS-PAGE. Prior to use, the PVDF membrane was activated by soaking it in methanol for a few seconds and afterwards, blot paper as well as the membrane were pre-equilibrated with transfer buffer. The transfer was carried out using the Bio-Rad Transblot Turbo System at a current of 0.6A and maximal voltage of $12 \mathrm{~V}$ for $20-30 \mathrm{~min}$, depending on the size of the respective proteins. Successful protein transfer was visualized by staining with Ponceau S solution, followed by destaining of the membrane with aqua bidest. In order to saturate free protein binding sites, membranes were incubated with blocking buffer for at least 30min according to manufacturer's instructions. Incubation with the respective primary antibody was performed rocking overnight at $4^{\circ} \mathrm{C}$ (see Table 7). The next day, the membranes were incubated with an appropriate secondary antibody for $45 \mathrm{~min}$ at room temperature. Membranes were washed four times with washing solution for $5 \mathrm{~min}$ and rinsed two times with aqua bidest. Antibody binding was detected using the WesternBreeze Chemiluminescent Immunodetection system, according to the manufacturer's instructions. The resulting chemiluminescent signal was detected with a luminescent image analyzer LAS-4000 (25sec - 1min using the Increment super setting).

\subsubsection{Membrane stripping}

Prior to reprobing, membranes were stripped in Roti ${ }^{\circledR}$-Free Stripping Buffer at $56^{\circ} \mathrm{C}$ for 30min, washed in PBS and blocked in blocking buffer for 30min at room temperature. For normalization, membranes were reprobed with anti- $\beta$-actin antibody as described in Chapter 3.5.4.

\subsection{Statistics and densiometric analysis}

Quantification of protein bands on western blots was performed using ImageJ with at least three independent experiments. Values of $\mathrm{n}$ refer to the number of mice in each group. In general, $\mathrm{p}$ values were determined by performing two-sided unpaired student's t-test using Microsoft Excel. A p value $<0.05$ was considered as statistically significant for all comparisons. 
Results

\section{Results}

In consideration of the very limited number of CEDNIK syndrome patients and the absence of a mouse model for this syndrome, physiologically relevant animal models would greatly facilitate the study of the role of SNAP29 during epidermal differentiation. In order to mimic the human CEDNIK syndrome in a mouse model and subsequently gain insight into the role of SNAP29 in epidermal differentiation, particularly in LB formation and maturation (see Chapter 1.3), we created two Snap29-deficient mouse models.

\subsection{Generation of total or epidermal Snap29-deficient mice}

Since no mammalian animal models are available for CEDNIK syndrome, alignment of human and mouse protein sequences revealed an overall conservation at the protein level of $83 \%$. Consequently, the mouse Snap29 gene can be considered to be the human SNAP29 homolog (see Figure 9).

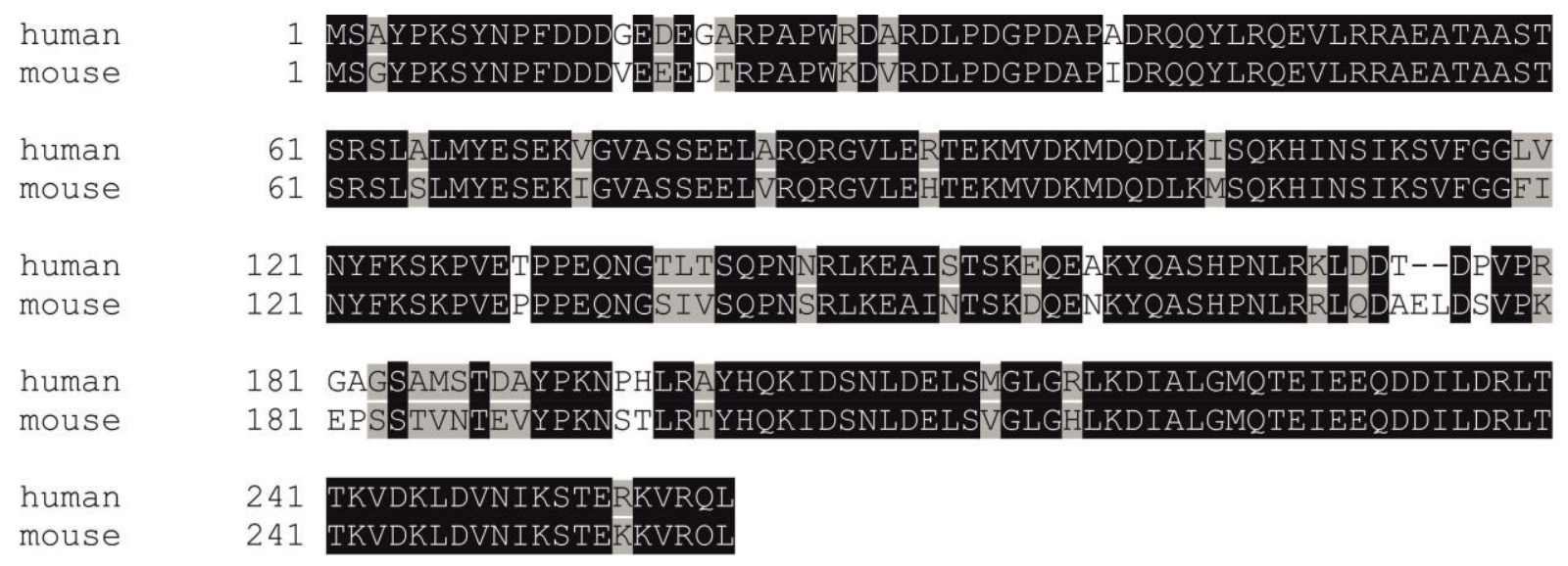

Figure 9: Alignments of murine SNAP29 with its human ortholog revealed $83 \%$ amino acid identity.

Comparison of the human (NCBI Reference Sequence: NM_004782.3) and murine (NCBI Reference Sequence: NM_023348.4) SNAP29 protein sequence. Identical (black) and conserved (grey) amino acid residues are indicated.

Snap29-deficient mice were generated using Cre mediated loxP recombination. Therefore, B6N;B6N-Snap29 ${ }^{\mathrm{tm} 1 \mathrm{a}(\mathrm{EUCOMM}) \mathrm{Wtsi}}$ heterozygous knock-in mice were derived from a mouse line generated by the Wellcome Trust Sanger Institute (WTSI) Mouse genetics programme (MGP) (see Figure 10 and Figure 11a). At first, mice were generated containing a floxed Snap29 allele (Snap29 $9^{\mathrm{fl} / \mathrm{wt}}$ ), and bred to homozygosity (Snap29 $9^{\mathrm{fl} / \mathrm{fl}}$ ). These animals showed no 
phenotypical abnormalities, indicating that the genetic manipulation did not affect the function of the Snap29 gene.

Total Snap29 (also referred to as Snap29-/) mice were generously provided by Prof. Dr. Nave from the Max Planck Institute (MPI) of Experimental Medicine in Göttingen (Department of Neurogenetics). Snap $29^{-/-}$mice were generated by intercrossing Snap $29^{\mathrm{f} / \mathrm{wt}}$ heterozygous knock-in mice with transgenic mice expressing a Cre recombinase under the control of the adenovirus EIIa promoter (Lakso et al, 1996). Snap29 $9^{\mathrm{fl} / \mathrm{wt}}$ mice harbor a FRT flanked lacZ/neomycin sequence, followed by a loxP site in intron 1-2 and an additional loxP site inserted downstream of exon 2 (see Figure 11). This promotor targets the expression of Cre recombinase to the early mouse embryo and EIIa gene products are ubiquitously expressed. In addition to the Cre-mediated excision of exon 2, the abnormal splicing of exon 1 to exon 3 results in a shift of the open reading frame with early termination at the eigth codon of exon 3 (see Figure 11b) (Schiller et al, 2015)

In order to further examine the role of SNAP29 in epidermal differentiation, a transgenic mouse line expressing Snap29 under the control of the keratinocyte specific keratin 14 (K14) promoter was generated (hereafter referred to as Snap $29^{\mathrm{fl} / \mathrm{l} l} / \mathrm{K} 14-\mathrm{Cre}$ mice) (see Figure 10). The K14 promoter is nearly exclusively expressed in basal keratinocytes (in addition to tongue, mouth, thymus and esophagus (Lloyd et al, 1995; Moll et al, 1982; Wang et al, 1997)). In addition, the K14 promoter is transcriptionally active in oocytes (Hafner et al, 2004), therefore, only male mice were taken to transmit the Cre gene, because in females Cre expression would result in recombination in oocytes (Scharfenberger et al, 2014). Prof. Dr. med. Sabine Eming from the Department of the University of Cologne generously provided us with transgenic mice expressing Cre recombinase under the control of the human K14 promotor. 
Results

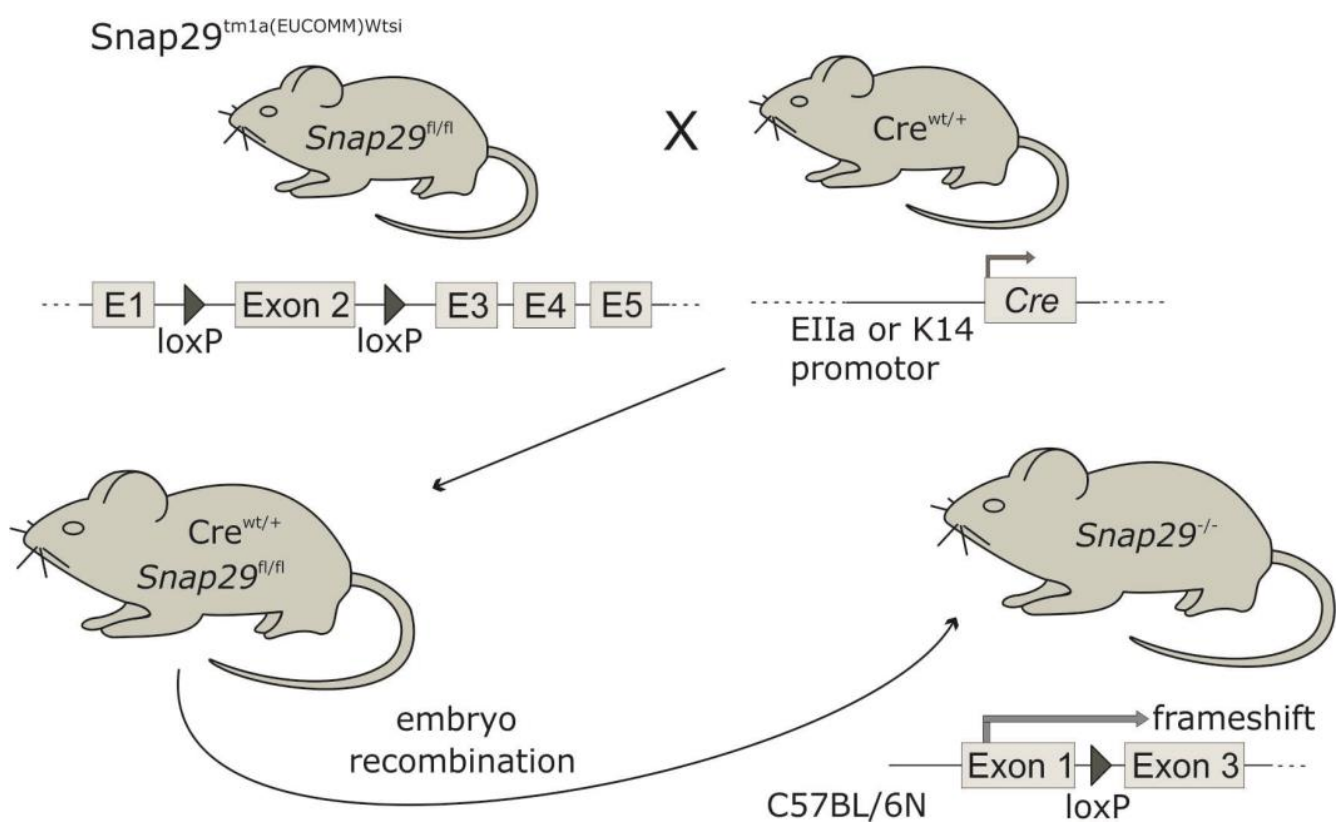

Figure 10: Schematic illustration of the generation of Snap29-deficient mice.

C57BL/6N mice carrying a floxed exon 2 of Snap29 were crossed with mice carrying a Cre recombinase gene under the control of the EIIa or K14 promoter, respectively. The Cremediated excision of the loxP-flanked exon 2 leads to an abnormal splicing of exon 1 to exon 3 and in a shift of the open reading frame with early termination after eight codons of exon 3. The illustration was kindly provided by Dr. Stina Schiller.

Moreover, the positive selection cassette can potentially interfere with normal expression of the targeted allele by disruption of normal splicing patterns, promoter interference, or premature transcript termination (Dragatsis \& Zeitlin, 2001). On this account, Snap $29^{\mathrm{fl} / \mathrm{fl}} / \mathrm{K} 14-$ Cre (epidermal Snap29 knockout) mice were generated by mating the Snap $29^{\mathrm{fl} / \mathrm{wt}}$ mouse line with a flippase (FLP) mouse line (B6.129S4-Gt(ROSA)26Sortm1(FLP1)Dym/RainJ, Jackson Laboratory), which expresses a variant of the FLP1 recombinase gene. By intercrossing animals with flippase knock-in mice, the FRT site-flanked LacZ-Neo cassette was excised (see Figure 11d/e). A Cre recombination event after intercrossing Snap2 $9^{\mathrm{f} / / \mathrm{wt}}$ mutants with transgenic mice expressing Cre under the control of the K14 promoter leads to an excision of Snap29 exon 2 exclusively in keratinocytes (Snap29 $9^{\mathrm{fl} / \mathrm{fl}} / \mathrm{K} 14-\mathrm{Cre}$; see Figure 11e/f), (Hafner et al, 2004). Ablation of the Snap29 gene products was verified using genomic DNA from tail tissue. 


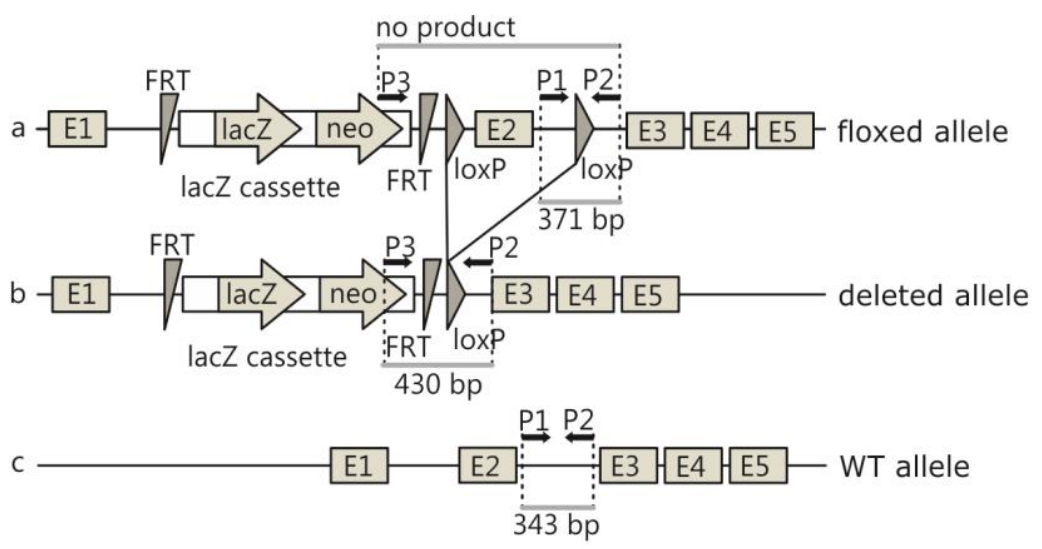

construction procedure and genotyping of Snap $29^{-\%}$ mice

construction procedure and genotyping of Snap $29^{\mathrm{f} / 1 / 1} / \mathrm{K} 14-\mathrm{Cre}$ mice
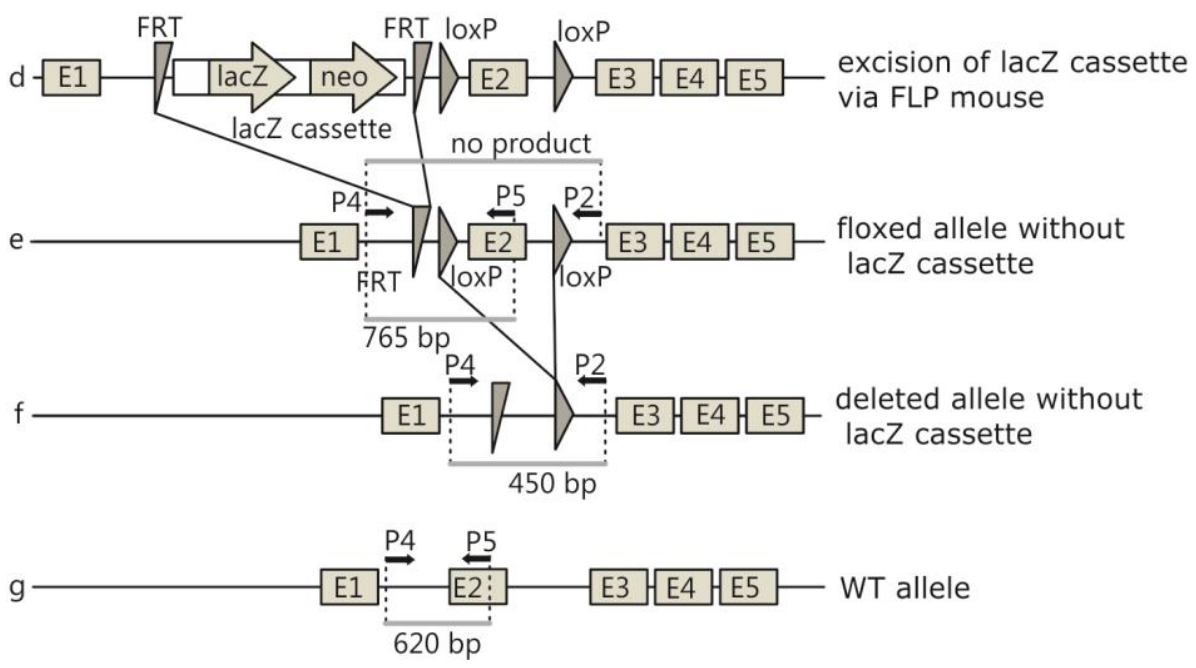

Figure 11: Allele constructs and strategies used for the generation of Snap29-- and Snap $29^{\mathrm{fl} / \mathrm{fl}} / \mathrm{K14}-\mathrm{Cre}$ mice (illustration modified from Schiller, Seebode et al. 2015).

(a) Floxed Snap $29^{\text {tmla(EUCOMM)Wtsi }}$ allele. (b) Snap29 allele after excision of exon 2. Complete loss of Snap29 function in Snap29-1- mice after mating Snap2 $9^{f / / w t}$ mice with mice expressing Cre under the control of the adenovirus EIIa promoter. (c) WT allele of the murine Snap29 gene. (d/e) Mating with a FLP mouse line leads to the excision of the lacZ cassette (f) Functional loss of Snap29 exclusively in keratinocytes (Snap29 $9^{\mathrm{fl} / \mathrm{fl}} / \mathrm{K} 14-\mathrm{Cre}$ mice) after mating with transgenic mice expressing Cre recombinase under the control of the keratinocyte specific K14 promoter. (g) WT allele of the murine Snap29 gene. Primers for genotyping are indicated as P1, P2, P3, P4, and P5, respectively (see Table 5). Grey boxes indicate exons, dark grey wedges the FRT sites, dark grey triangles the loxP sites, grey arrows the lacZ and neomycin cassette. 


\subsection{Confirmation of a functional Snap29 knockout}

\subsubsection{Genotyping of Snap29-deficient mice}

At the beginning of this thesis, Snap $29^{-/-}$mice were generated, followed by the generation of Snap $29^{\mathrm{fl} / \mathrm{fl}} / \mathrm{K} 14-\mathrm{Cre}$ mice in order to analyze the role of SNAP29 during epidermal differentiation. At weaning, no animals with Snap29 deficiency were identified. In order to further investigate whether Snap29 is crucial for embryonic survival, pubs were sacrificed by decapitation directly after birth, resulting in P0 neonates with the following genotypes: Snap $29^{\mathrm{wt} / \mathrm{wt}}$, EIIa-Cre/Snap29 $9^{\mathrm{wt} / \mathrm{wt}}$, Snap $29^{\mathrm{wt} / \mathrm{fl}}$, EIIa-Cre/Snap29 $9^{\mathrm{wt} / \mathrm{fl}}$, Snap29 $9^{\mathrm{fl} / \mathrm{fl}}$ and EIIaCre/Snap29 $9^{\mathrm{fl} / \mathrm{fl}}\left(\right.$ Snap29 $\left.9^{-/}\right)$. The ratio of newborn Snap29-deficient mice (Snap29-/-) was in close agreement with the expected for Mendelian inheritance. Epidermal knockout mice were also born at the expected Mendelian ratio, indicating that disruption of Snap29 did not result in embryonic lethality. The genotype of the animals was determined by PCR analysis of genomic DNA extracted from tail biopsies (see Figure 12). In the offspring, the samples were collected after weaning (see Chapter 3.2 and 3.4.4).

Primers P1, P2, and P3 were used for genotyping Snap $29^{-/-}$mice. For primer sequences see Table 5. Wild-type (WT) Snap29 alleles showed an amplification product with a size of $343 \mathrm{bp}$, whereas floxed Snap29 alleles showed a band with a higher size of $371 \mathrm{bp}$ due to the inserted loxP sites (primer P1 and P2). Heterozygous mice could be identified by the presence of both amplification products, visualized as double bands. The presence of a Cre knock-in could be identified by using the primer pair EIIa-Cre-s and EIIa-Cre. As a result of recombination, an amplification product of 430bp was detected (primer P2 and P3), and therefore confirming the excision of exon 2 (see Figure 11Figure 12).

Primers P4, P5, and P2 were used for genotyping Snap29 ${ }^{\mathrm{fl} / \mathrm{fl}} / \mathrm{K} 14-\mathrm{Cre}$ mice. WT Snap29 alleles showed an amplification product with a size of 620bp, floxed Snap29 alleles showed a band with a higher size of 765bp (primer P4 and P5), respectively. After recombination, an amplification product of 450bp can be detected (primer P2 and P4). Primer K14-Cre-up and K14-Cre-down were used for genotyping K14-Cre mutant mice. 


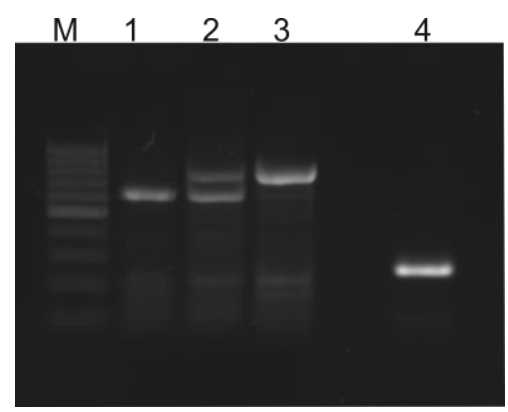

Figure 12: Representative PCR products of genomic DNA isolated from tail biopsies from Snap29 $9^{\mathrm{fl} / \mathrm{fl}} / \mathrm{K} 14-$-Cre mice.

Analysis of the Snap29 alleles: (1) WT Snap29 allele (620bp), (2/3) lower band represents the WT Snap29 allele, upper band represents the floxed Snap29 allele (765bp), (4) band of the inserted Cre recombinase (250bp). Lane 1: WT, lane 2: Snap29 ${ }^{\mathrm{fl} / \mathrm{wt}}$, lane 3: Snap $29^{\mathrm{fl} / \mathrm{fl}}$, lane 4: inserted Cre recombinase, marker: 100bp.

\subsubsection{Quantitative Real-time PCR, Western Blot, and immunohistochemical staining}

In order to examine Snap29 gene expression in mutant mice and to further exclude a residual Snap29 activity due to an incomplete Cre-mediated gene inactivation, quantitative Real-time PCR was performed using mRNA derived from fibroblasts of Snap29-/- mice and control littermates as described in Chapter 3.4.5. Relative quantification of Snap29 expression levels revealed no Snap29 mRNA expression in fibroblasts of Snap $29^{-/-}$mice compared to WT cells (see Figure 13). In fibroblasts derived from heterozygous littermates, Snap29 expression reached about approximately $50 \%$ of the WT level, demonstrating the absence of dose compensation.

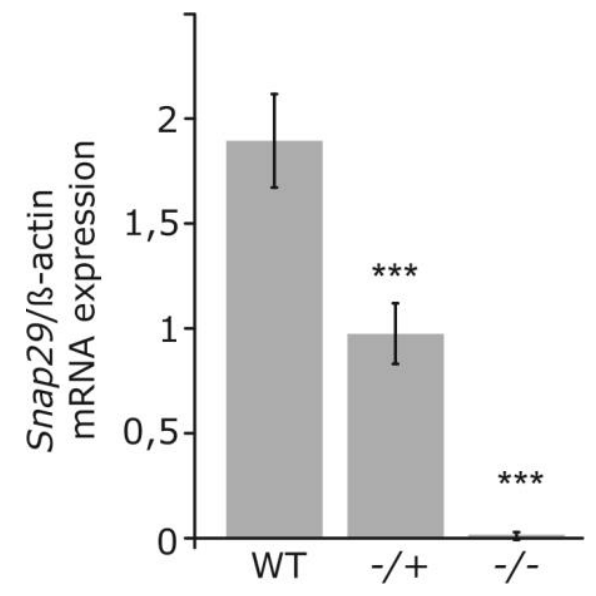

Figure 13: Snap29 ablation in fibroblasts derived from Snap29 ${ }^{-/-}$mice.

Comparison of Snap 29 mRNA expression levels from fibroblasts obtained from control mice (WT), Snap $29^{\mathrm{fl} / \mathrm{wt}} / \mathrm{EIIa}-\mathrm{Cre}$, and Snap $29^{-/-}$mice. Expression levels are shown as absolute values. $\beta$-actin was used as the internal control gene to normalize the expression of the target gene. Results represent mean values from three independent experiments with $n=3$. Error bars indicate error propagation. For calculation of statistical significances the student's t-test was used $(* * *=p<0.001)$ (Schiller, Seebode et al, 2015). 
In addition, efficiency of the Snap29 knockout construct was assessed on protein level via western blot analysis and immunohistochemical staining, respectively (see 3.4.8.3 and 3.5). Western blot analysis was performed using lysates of fibroblasts and whole skin protein extracts obtained from Snap29-/- mice (see Chapter 3.5.1). SNAP29 was detected in extracts from WT and heterozygous mice, whereas the immunoblot showed a $63 \%$ reduction of SNAP29 levels in fibroblasts of heterozygous mice as compared to control littermates. In the skin of Snap29-/- mice, SNAP29 was below limits of detection, verifying the total lack of SNAP29 protein (see Figure 14). The immunoblot with whole skin extracts showed an additional band at $35 \mathrm{kDa}$, which is presumably a non-specific band. The smaller amount of protein in heterozygous mice is consistent with results of quantitative real-time PCR analysis (see Figure 13), which indicates inactivation of one Snap29 allele.

a

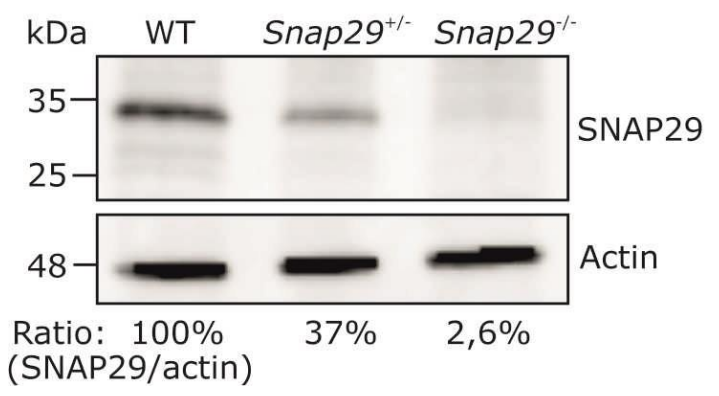

b

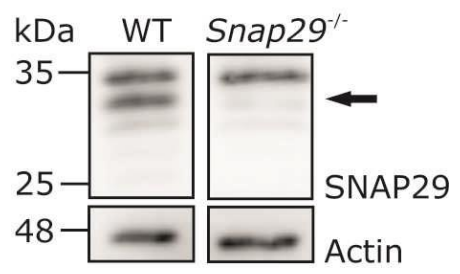

\section{Figure 14: Disruption of SNAP29.}

(a) Western blot analysis: Equal amounts of protein $(25 \mu \mathrm{g})$ of fibroblast extracts obtained from control mice, Snap $29^{\mathrm{fl} / \mathrm{wt}} / \mathrm{EIIa}-\mathrm{Cre}$, and Snap $29^{-/-}$mice were separated by SDS-PAGE and analyzed by western blot analysis using anti-SNAP2 9 and anti- $\beta$-Actin antibody, respectively (see Table 7). The distinct protein band of approximately $29 \mathrm{kDa}$ vanished in homozygous mutant mice. (b) Equal amounts of whole skin protein extracts of control and Snap $29^{- \text {- }}$ dorsal skin samples were separated by SDS-PAGE and examined by western blot analysis using anti-SNAP29 and anti- $\beta$-actin antibody, respectively (see Table 7). A distinct protein band of approximately $29 \mathrm{kDa}$ was exclusively visible in control mice skin. The immunoblot showed an additional band at $35 \mathrm{kDa}$, which is presumably a non-specific band. The SNAP29 blot is depicted on top; $\beta$-actin for normalization is displayed on the bottom. These blots are representative for at least two independent experiments showing similar results. The western blot band intensities were measured with ImageJ software (Schiller, Seebode et al, 2015). 
Immunostaining against SNAP29 of skin sections derived from Snap29 $9^{-/}$showed no staining throughout the entire skin sample (see Chapter 3.4.8.3). As expected, SNAP29 positive cells were observed in the dermis of Snap $29^{\mathrm{fl} / \mathrm{fl}} / \mathrm{K} 14-\mathrm{Cre}$ mice, but immunostaining of skin sections of Snap $29^{\mathrm{fl} / \mathrm{fl}} / \mathrm{K} 14-\mathrm{Cre}$ showed a deficiency of SNAP29 exclusively in the epidermis (see Figure 15).

Furthermore, keratinocytes isolated from Snap29 $9^{\mathrm{fl} / \mathrm{fl}} / \mathrm{K} 14-\mathrm{Cre}$ mice were immunostained against SNAP29 (see Chapter 3.4.8.4). Positive staining was detected in keratinocytes of control mice, whereas no staining was observed in Snap29-deficient keratinocytes (see Figure 16). In concordance with data from other authors, SNAP29 was mostly localized to the perinuclear region in control cells (Fuchs-Telem et al, 2011; Rapaport et al, 2010; RotemYehudar et al, 2001).

As a result, the acquired data confirm a complete absence, or at least significant decrease, of Snap29 expression in the skin of mutant mice.

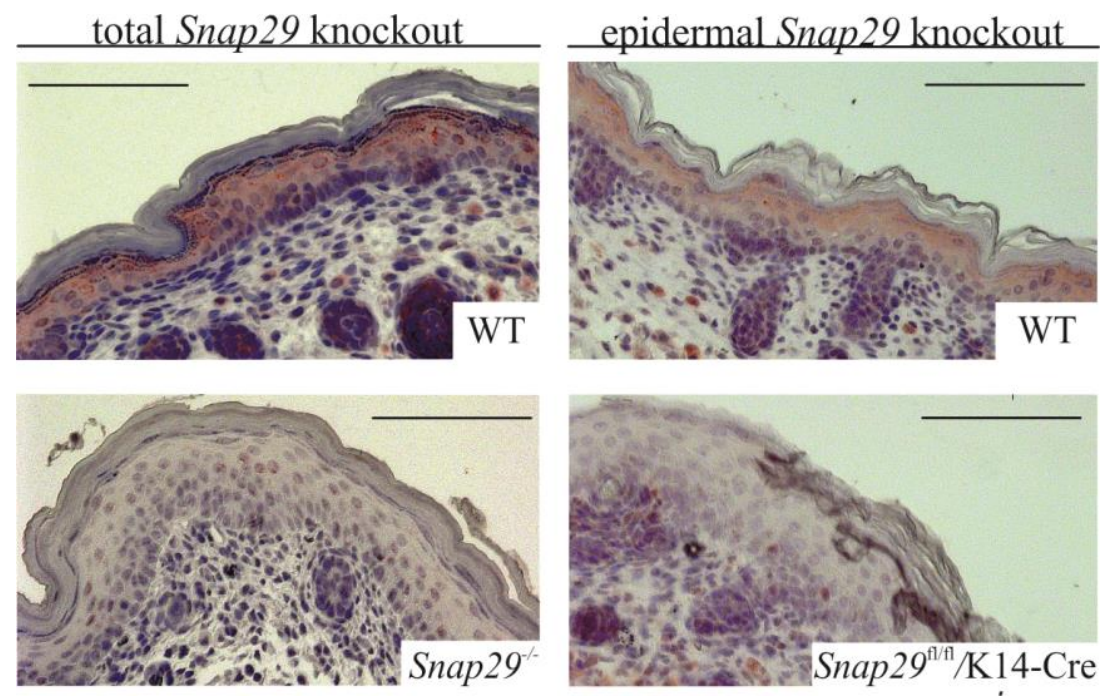

Figure 15: Immunostaining against SNAP29.

Representative, immunohistochemical staining against SNAP29 in dorsal skin sections derived from neonatal Snap $29^{-/-}$, Snap $29^{\mathrm{fl} / \mathrm{fl}} / \mathrm{K} 14-\mathrm{Cre}$ mice and control littermates verified the absence of SNAP29 mutant epidermis or whole skin sections. Scale bars: 100 $\mu$ m (Schiller, Seebode et al, 2015). 

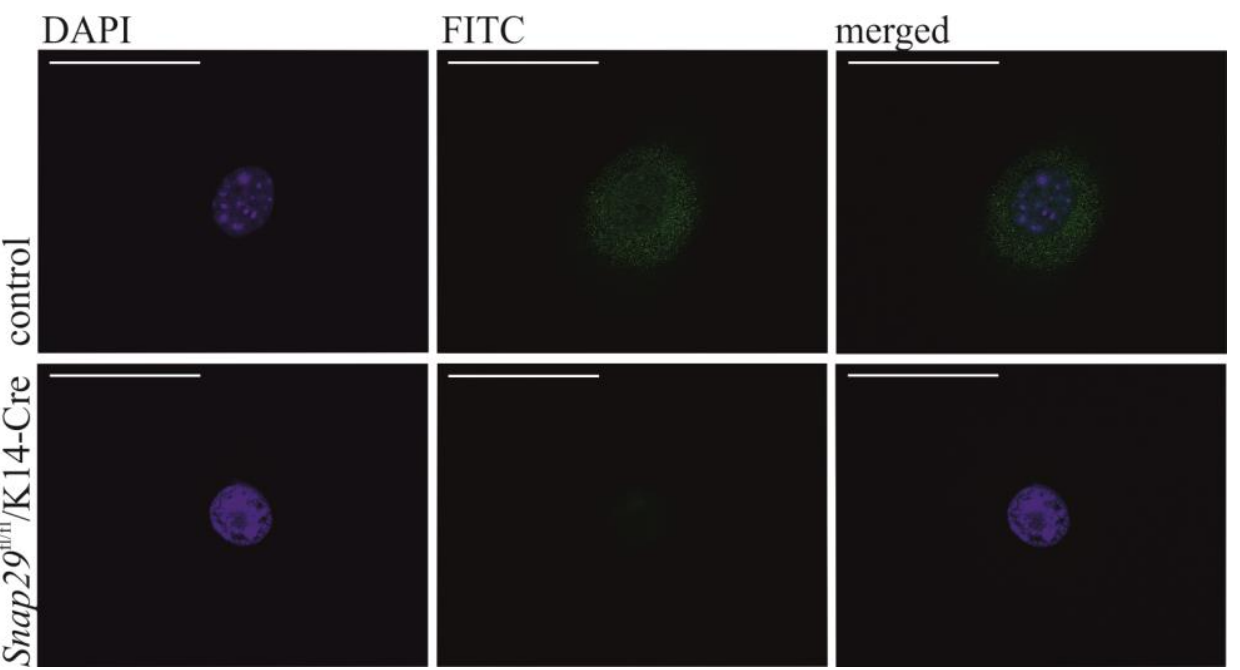

Figure 16: Immunofluorescence staining against SNAP29 in keratinocytes.

Immunofluorescence staining for SNAP29 (green) was carried out on keratinocytes derived from Snap $29^{\mathrm{fl} / \mathrm{fl}} / \mathrm{K} 14-C r e$ mice and control littermates. Positive staining was detected in keratinocytes from control mice and no staining in cells from Snap $29^{\mathrm{fl} / \mathrm{fl}} / \mathrm{K} 14-\mathrm{Cre}$ mice. Representative stainings are shown. Nuclei were counterstained with Hoechst 33342 (blue). Scale bars: $50 \mu \mathrm{m}$.

\subsection{Snap29 deficient mice exhibit a distinct ichthyotic phenotype and neonatal lethality}

Immediately after birth, Snap $29^{-/-}$mice showed a distinct ichthyotic phenotype with taut skin and ichthyotic lesions (see Figure 17), whereas Snap $29^{+/-}$heterozygous pups were phenotypically indistinguishable from control littermates.

Snap29-deficient pups were occasionally found in the first few hours after birth. However, they died shortly after birth, within one to four hours or they were eaten by the mother. Animals with Snap29-deficiency exhibited a slightly reduced body size and extensive abnormalities in skin morphology (see Figure 17). The ichthyosiform phenotype was characterized by taut skin, which was particularly unwrinkled, scaly and stretched very tightly (Schiller, Seebode et al, 2015). Therefore, the animals were unable to extend their limbs. Concordantly, no suckling behavior was observed and none of the mutant mice contained milk in their stomachs.

Due to the neonatal lethality of Snap $29^{-/-}$mice, Snap2 $9^{\mathrm{fl} / \mathrm{fl}} / \mathrm{K} 14-\mathrm{Cre}$ mice were generated, in order to circumvent the early lethality and to further examine the role of SNAP29 in epidermal differentiation in adult mice. Unfortunately, epidermal Snap29-deficient mice 
exhibited the same distinct ichthyotic phenotype (see Figure 17) and neonatal lethality as previously described in the Snap $29^{-/-}$mice.

Adult Snap $29^{\mathrm{fl} / \mathrm{wt}} / \mathrm{EIIa}-\mathrm{Cre}$ and Snap29 $9^{\mathrm{fl} / \mathrm{wt}} / \mathrm{K} 14-$ Cre mice had no apparent phenotype, showed no abnormalities in motor behaviour and were healthy and fertile.
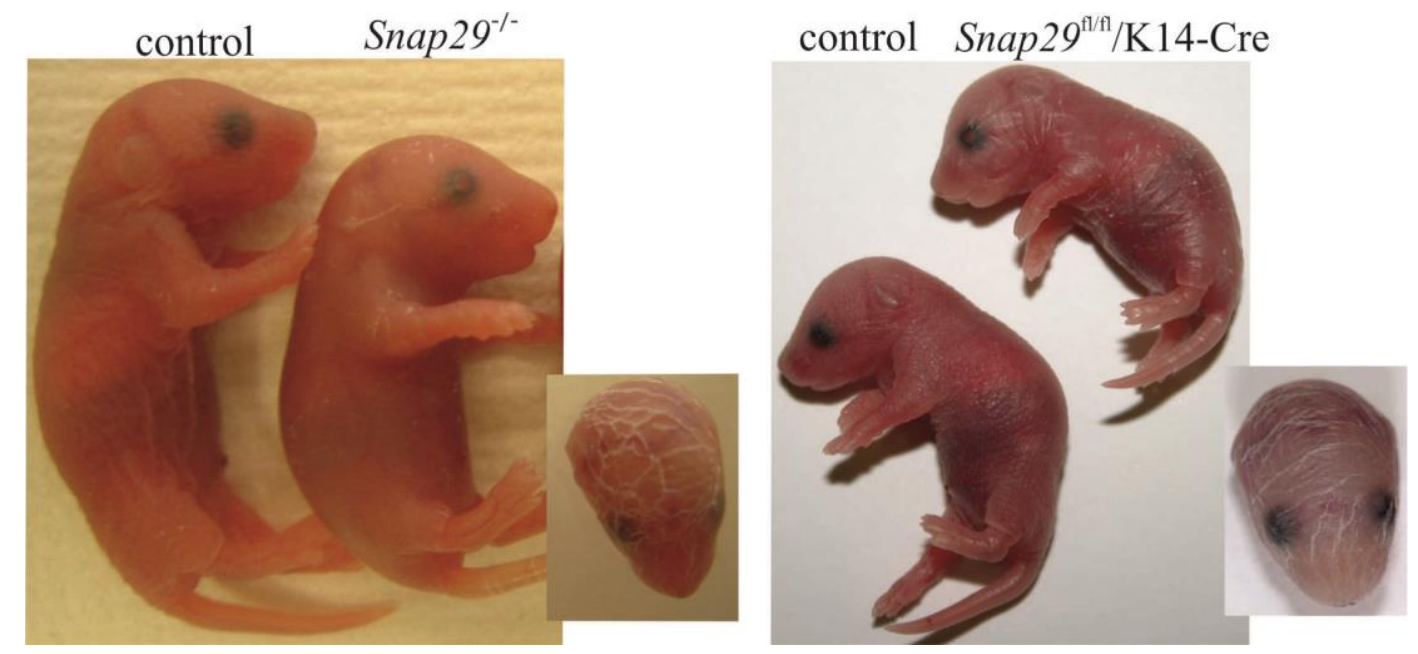

Figure 17: Ichthyotic phenotype of Snap29-deficient mice.

Macroscopic appearance of Snap $29^{-/-}$Snap $29^{\mathrm{fl} / \mathrm{fl}} / \mathrm{K} 14-\mathrm{Cre}$ mice, and control littermates. Snap29-deficient newborns died shortly after birth. The mutant mice showed a smaller size and a cutaneous phenotype with taut and scaly skin. Newborns were separated from their mother immediatly after birth and photographed. Representative pictures are shown (Schiller, Seebode et al, 2015).

\subsection{Snap29 deficiency causes altered histomorphological features in mouse skin.}

After confirming the functional knockout, the impact of the Snap29 knockout on epidermal differentiation was analyzed using immunohistohemistry as described in chapter 3.4.8.3. A histological analysis of skin sections derived from Snap2 $9^{\mathrm{fl} / \mathrm{fl}} / \mathrm{K} 14-\mathrm{Cre}$ and Snap29/- neonates stained with hematoxylin and eosin (see Chapter 3.4.8.2) revealed noticeable disturbances in the epidermal structure (see Figure 18a). All epidermal cell layers were apparent in both Snap29-deficient mouse lines. However, the suprabasal skin layers of Snap29-deficient mice exhibited an intensive thickening. Healthy epidermis of newborn mice consisted of two or three living layers above the stratum basale. Epidermal as well as total Snap29-deficient murine epidermis revealed additional suprabasal cell layers (acanthosis) (see Figure 18b). The stratum corneum of control mice epidermis showed the characteristic basket-weave-like 
appearance (Elias, 2012). However, the stratum corneum of Snap29-deficient mice was thickened and its structure was condensed (hyperkeratosis), indicating disturbances in the formation of lipid lamella as well as the inability of Snap29-deficient keratinocytes to shed off properly during desquamation. The number of keratohyalin granules normally formed in the upper granular and transitional layer of the epidermis was decreased in both mutant mice (see Figure 18a, white arrows). The number of hair follicles was evidently reduced compared to control mice. In addition, there was a stronger reduction of hair follicles in Snap29-/ newborns (50\% reduction) compared to Snap $29^{\mathrm{fl} / \mathrm{fl}} / \mathrm{K} 14-\mathrm{Cre}$ mice (19\% reduction). In contrast to Snap $29^{\mathrm{fl} / \mathrm{fl}} / \mathrm{K} 14-\mathrm{Cre}$ mice, only Snap $29^{-/-}$mice revealed remnants of nuclei in the cornified layers (parakeratosis) (see Figure 18a, black arrows).

Adult Snap $29^{\mathrm{fl} / \mathrm{wt}} / \mathrm{EIIa}-\mathrm{Cre}$ and Snap $29^{\mathrm{fl} / \mathrm{wt}} / \mathrm{K} 14-C r e$ mice showed no overt histological abnormalities.

Taken together, both mutant mouse lines showed epidermal thickening, hyperkeratosis, acanthosis and a reduced number of keratohyalin granules as well as hair follicles. The only histomorphological difference between both lines was the presence of a parakeratosis as well as an increased reduction of hair follicles in total knockout mice. 
a

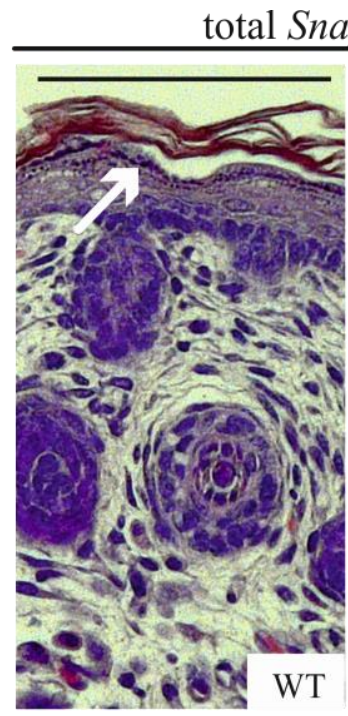

29 knockout

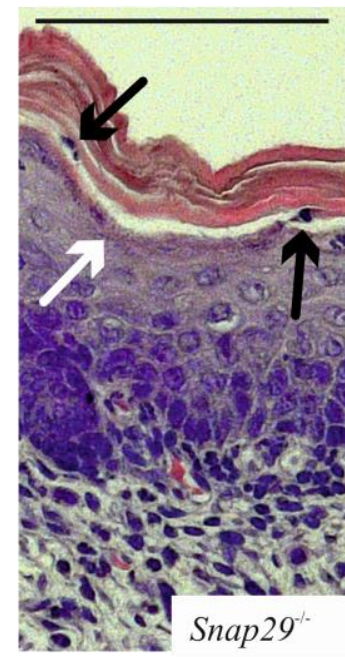

epidermal Snap29 knockout

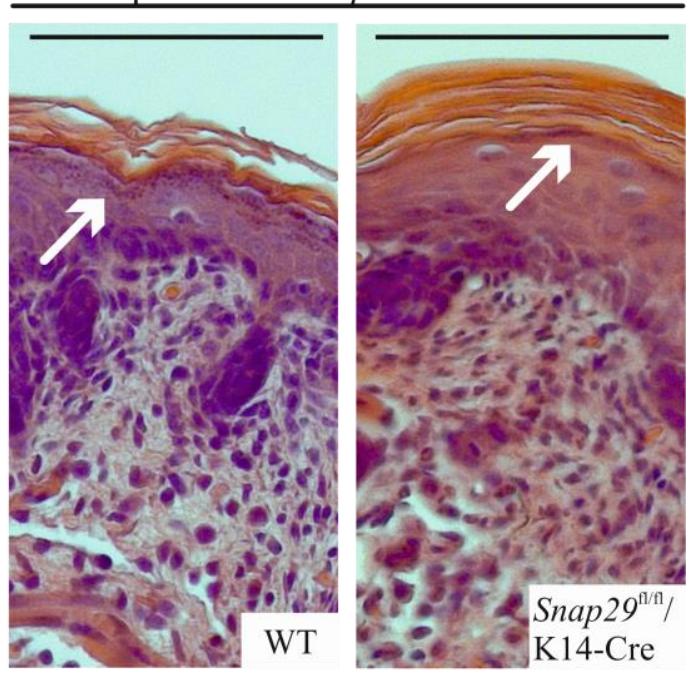

$\mathrm{b}$

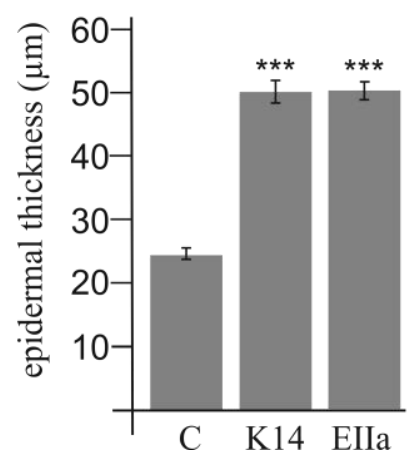

Figure 18: Comparison of skin morphology of Snap29-deficient and WT mice.

(a) Representative hematoxylin and eosin stainings of skin sections from newborn control, Snap $29^{-/-}$and Snap $29^{\mathrm{fl} / \mathrm{fl}} / \mathrm{K} 14-\mathrm{Cre}$ mice showed an overview of the altered histomorphological features in Snap29-deficient epidermis. Control epidermis revealed the characteristic basketweave conformation of the stratum corneum. Compared to control mice, the suprabasal layers in mutant mice epidermis were thickened. The stratum corneum was thickened and condensed (hyperkeratosis). The number of keratohyalin granules (white arrows) and hair follicles were reduced. Retention of nuclei in the stratum corneum (parakeratosis) was exclusively visible in Snap 29 ${ }^{-/}$mice (black arrows). Scale bars: $100 \mu \mathrm{m}$. (b) Quantification of acanthosis in the viable epidermis (three animals per group, three fields per animal). Data are shown as mean, error bars indicate SEM. For calculation of statistical significances the student's t-test was used $(* * *=p<0.001)$. C: control, K14: Snap29 $9^{\mathrm{fl} / \mathrm{fl}} / \mathrm{K} 14-\mathrm{Cre}$, EIIa: Snap29 ${ }^{-/-}$(Schiller, Seebode et al, 2015).

\subsection{Keratinocyte differentiation is disturbed in mice lacking SNAP29.}

During epidermal differentiation proliferating basal keratinocytes withdraw from the cell cycle to move upwards towards the surface and continuously differentiate until they become keratin-filled enucleated corneocytes, which finally shed off during desquamation (see Chapter 1.2.5). 
In order to examine the consequences of Snap29 deletion at the level of epidermal differentiation, skin samples derived from epidermal as well as total knockout mice were stained with several differentiation markers using immunohistochemistry and immunofluoresence as described in Chapter 3.4.8.3 and 3.4.8.4. Among specific markers for epidermal differentiation are early markers, such as K14 and K10, and later markers, such as filaggrin and involucrin (see Chapter 1.2.1). In control epidermis K14 expression was restricted to the basal layer of the epidermis, but was additionally present in all suprabasal layers in Snap29-deficient skin (see Figure 19), indicating abnormal keratinocyte differentiation. K10 is normally expressed in the postmitotic layers of the stratum spinosum and stratum granulosum in healthy skin. Immunohistochemistry revealed no differences in the amount or the distribution of the suprabasal cell-specific K10 (see Figure 19). Furthermore, double immunofluorescence staining of K10 and K14 revealed an increase in keratinocytes of the basal and the spinous layer simultaneously expressing both keratins in Snap29-/- and in

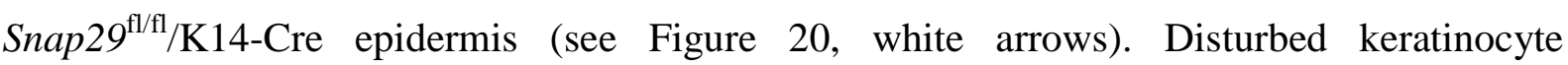
differentiation was additionally demonstrated by the presence of nuclear remnants in the stratum corneum of total knockout mice (see Figure 18).

In conclusion, these findings indicate a disturbed differentiation of keratinocytes in mutant epidermis.
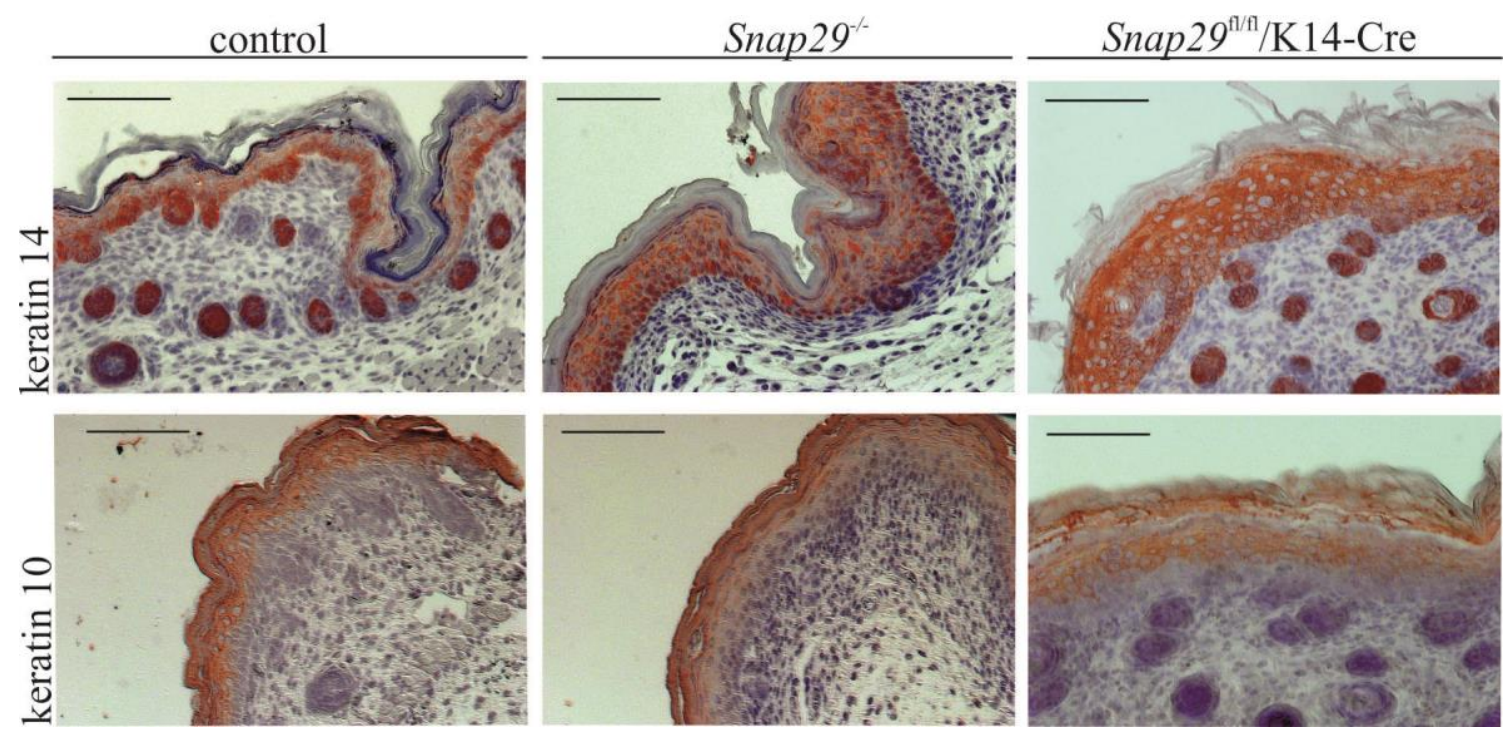

Figure 19: Analysis of early and late differentiation markers.

Immunohistochemical staining of dorsal skin samples derived from Snap29-/- Snap $29^{\mathrm{fl} / \mathrm{fl}} / \mathrm{K} 14-$ Cre and control littermates with antibodies against K10 and K14 (see Table 7). K14 was expressed in all living layers of mutant epidermis. No differences in K10 expression or distribution were detectable. Representative stainings are shown. Scale bars: $100 \mu \mathrm{m}$. (Schiller, Seebode et al, 2015). 
Results
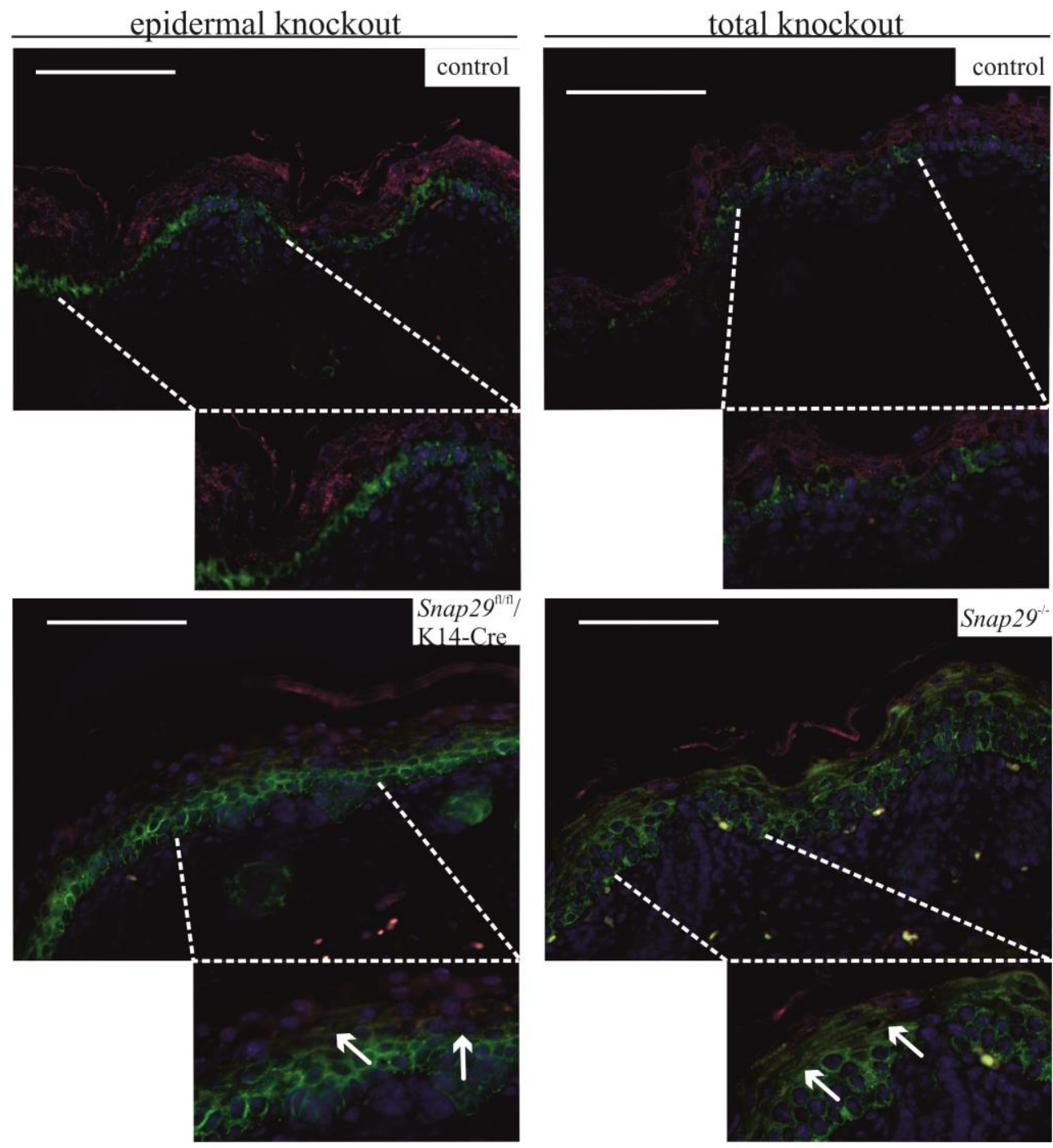

\section{Figure 20: Immunofluorescence double staining with early and late differentiation} markers.

Co-expression of K10 (red) and K14 (green) in mutant epidermis (white arrows), especially in spinous cells. Dorsal skin samples were derived from Snap $29^{-/-}$, Snap $29^{\mathrm{fl} / \mathrm{fl}} / \mathrm{K} 14-$ Cre mice and control littermates. Panels below represent higher magnification views of the marked areas in the upper panel and nuclei were counterstained with DAPI (blue). Representative stainings are shown. Scale bars: $100 \mu \mathrm{m}$.

Since keratinocyte differentiation was disturbed (see Figure 19) and nuclear remnants were observed in the stratum corneum of total knockout mice (see Figure 18a), we further examined structural proteins necessary for epidermal barrier formation by immunohistochemistry (see Chapter 3.4.8.3). During corneocyte maturation, the CE formation relies on intracellular synthesis of structural proteins such as filaggrin, which functions to aggregate keratin intermediate filaments into macrofibrills in the developing corneocytes. Filaggrin is further processed into free amino acids forming natural moisturizing factors (NMFs), which protect the epidermis from water loss (see Chapter 1.2.2). 
We found reduced levels of filaggrin, and since profilaggrin is the main component of keratohyalin granules (see Chapter 1.2.1), the number of these granules was also decreased in the upper stratum granulosum of Snap $29^{-/-}$epidermis compared to control epidermis (as already shown, see Figure 18a) (see Figure 21). Immunostaining of Snap29 $9^{\mathrm{fl} / \mathrm{fl}} / \mathrm{K} 14-\mathrm{Cre}$ epidermis showed the same results (Schiller, Seebode et al, 2015). Involucrin, another structural protein of the CE, is expressed in the suprabasal layers of the epidermis (Kalinin et al, 2002). During early CE assembly, it becomes aligned to the plasma membrane, which is subsequently replaced by the CE (see Chapter 1.2.2). Involucrin levels were decreased in the cells of the upper stratum spinosum and stratum granulosum, indicating disturbed keratinization.

In conclusion, the abnormal formation of suprabasal layers in mutant mouse epidermis was associated with apparent changes in levels of epidermal differentiation markers together with abnormal corneocyte formation.
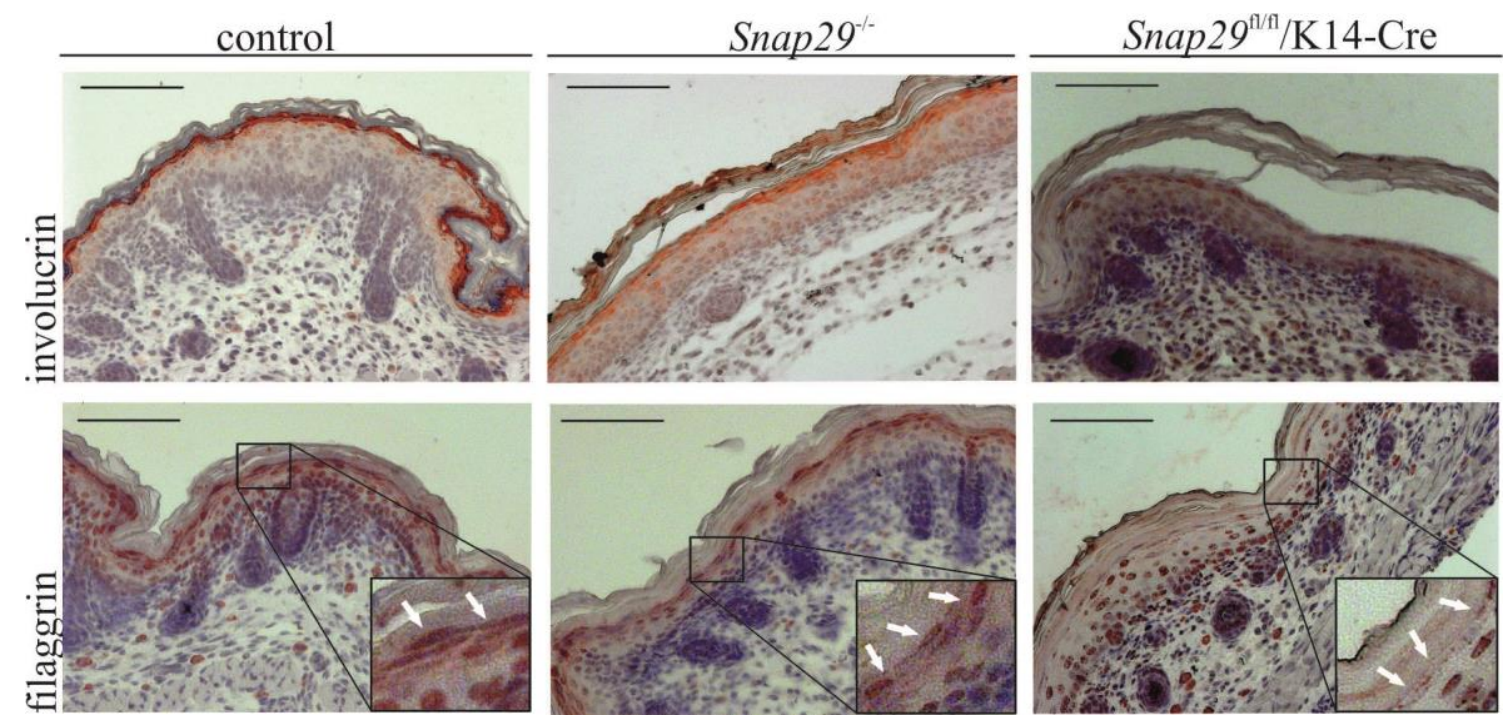

\section{Figure 21: Analysis of late differentiation markers.}

Representative images of immunohistochemical stainings of dorsal skin sections from newborn control and Snap29-deficient mice for filaggrin and involucrin (see Table 7). Protein levels of involucrin and filaggrin were reduced in both mutant mice epidermis. The number of keratohyalin granules (white arrows) was reduced in the epidermis of Snap29 $9^{-/-}$and Snap $29^{\mathrm{fl} / \mathrm{fl}} / \mathrm{K} 14-\mathrm{Cre}$ mice, as previously shown (see Figure 18). Panels below represent higher magnification views of the marked areas in the upper panel. Scale bars: $100 \mu \mathrm{m}$. (Schiller, Seebode et al, 2015). 


\subsection{Snap29 deficiency causes enhanced levels of epidermal proliferation.}

In order to determine whether the epidermal thickening was caused by increased cell proliferation, immunostaining against Ki67 was performed (see Chapter 3.4.8.3, Table 7). Ki67 is a commonly used marker for cell proliferation and the antigen is confined to the nucleus during interphase, whereas in mitosis the protein is relocated to the surface of the chromosome. Ki67 is present during all active phases of cell cycle (G1, S, G2, and mitosis), while it is absent in resting cells (G0 phase) (Scholzen \& Gerdes, 2000). The number of Ki67positive cells was determined by enumeration, which revealed an increased number of Ki67positive cells in both mutant epidermises as compared to controls. Control epidermis showed $36 \%$ of positive nuclei, whereas $52 \%$ were detected in Snap $29^{-/-}$epidermis and $56 \%$ in Snap $29^{\mathrm{fl} / \mathrm{fl}} / \mathrm{K} 14-\mathrm{Cre}$ epidermis, respectively (see Figure 22). Furthermore, Ki67-positive cells were restricted to the basal cells in control epidermis, whereas Ki67-staining was occasionally detected in the suprabasal layers of mutant skin, which is often observed in hyperproliferative skin disoders (see Figure 22) (Merritt et al, 2002; Potten, 1981).

Given an increased number of Ki67-positive cells in mutant epidermis, a possible counterbalance between cell proliferation and apoptosis was investigated. Therefore, immunohistochemical staining against the activated form of the apoptotic mediator caspase 3 was performed (see Table 7). In healthy skin, caspase 3 is not active during epidermal differentiation (Lippens et al, 2000) and no caspase 3-positive cells were detected in control and mutant epidermis of Snap $29^{\mathrm{fl} / \mathrm{fl}} / \mathrm{K} 14-\mathrm{Cre}$ mice (see Figure 23). Immunostaining of Snap $29^{-/}$K14-Cre epidermis showed the same results. In conclusion, no alteration in apoptotic activity was detected in the epidermis of Snap29-deficient mice (Schiller, Seebode et al, 2015).

Expression of K6 indicates disturbances in epidermal homeostasis during wound healing or is a consequence of hyperproliferative conditions (see Chapter 1.2.1). In healthy epidermis K6 expression is restricted to the cells of the hair follicles (Stoler et al, 1988). Consistently with Ki67 staining, pronounced levels of K6 were observed in all viable cell layers of Snap29deficient mice, but not in control mice (see Figure 22a).

Taken together, these observations suggest that the hyperkeratotic phenotype of Snap29deficient mice might occur as a result of increased cell proliferation in the basal epidermal layer. 
a
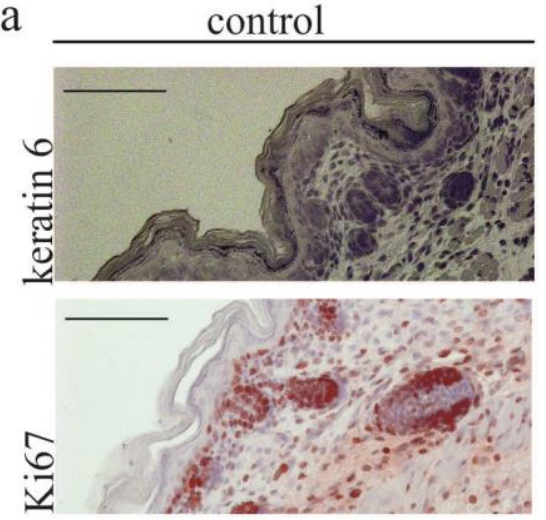

b

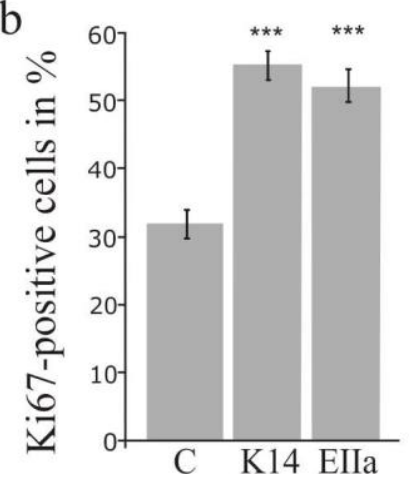

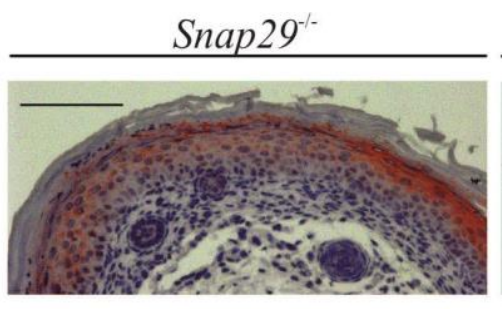

$\operatorname{Snap} 29^{\mathrm{n} / 1 / 1} / \mathrm{K} 14-\mathrm{Cre}$
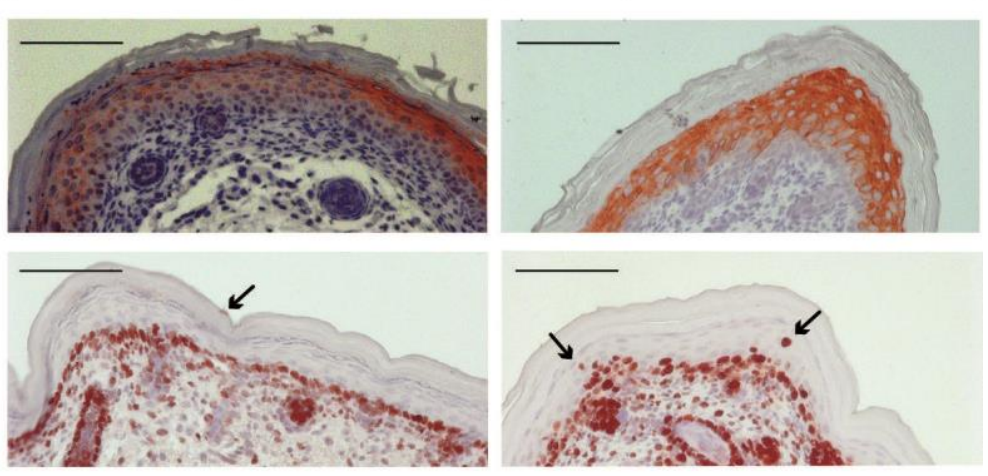

\section{Figure 22: Hyperproliferative conditions in Snap29-deficient mice.}

(a) Representative immunohistochemical stainings of dorsal skin sections of Snap29-/-, Snap $29^{\mathrm{fl} / \mathrm{fl}} / \mathrm{K} 14-\mathrm{Cre}$ mice and control littermates with antibodies against the proliferation markers K6 and Ki67 (see Table 7). Expression of K6 was strongly increased in Snap29deficient epidermis. An enlarged number of Ki67-positive cells was detected by enumeration. Ki67-positive cells were also detectable in subrabasal layers in both mutant mice (black arrows). Scale bars: $100 \mu \mathrm{m}$. (b) Quantification of Ki67 positive cells in the viable epidermis (500 cells enumerated). The plot represents the mean of three independent experiments, error bars indicate SEM. For calculation of statistical significances the student's t-test was used

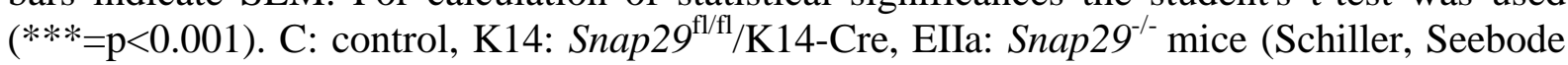
et al, 2015).
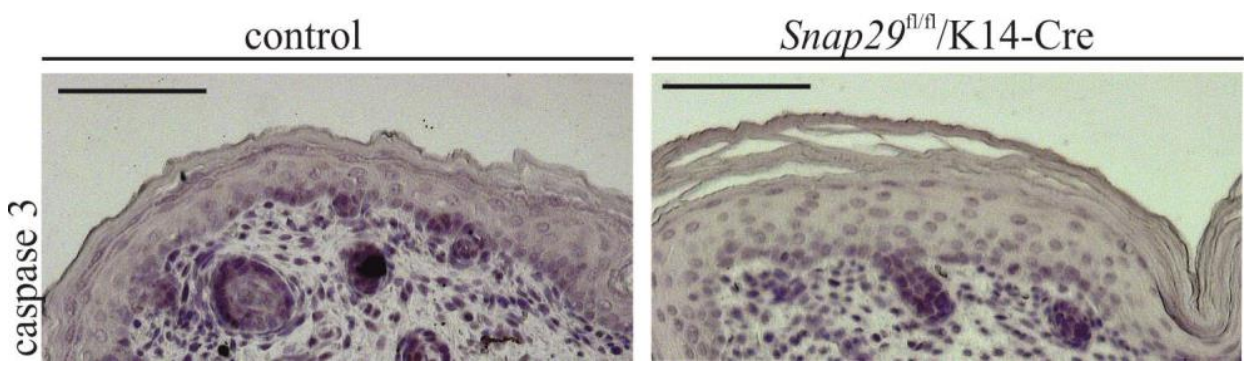

Figure 23: No detection of apoptotic activity in Snap29-deficient mice.

Representative immunohistochemical staining of epidermal dorsal sections of Snap $29^{\mathrm{fl} / \mathrm{fl}} / \mathrm{K} 14-$ Cre mice with an antibody against the active form of caspase 3. No caspase 3-positive cells were detected in control and mutant epidermis. Scale bars: $100 \mu \mathrm{m}$. 


\subsection{Ultrastructural analysis of Snap29-deficient epidermis reveals altered formation and maturation of LBs.}

The cutaneous phenotype of the human CEDNIK syndrome is associated with a markedly impaired maturation and secretion of LBs (Sprecher et al, 2005) (see Chapter 1.3). In order to investigate whether the formation as well as maturation and secretion of LBs is also disturbed in our mouse models, ultrastructural analyses of murine epidermis were performed (see Chapter 3.4.8.8). In accordance with the human CEDNIK syndrome, EM images of skin

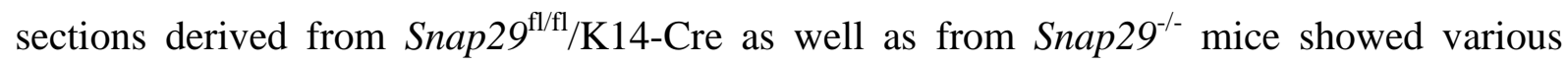
epidermal anomalies.

The stratum corneum of control mice showed a loose, basket-weave structure, however both mutants revealed a condensed stratum corneum with compressed corneocyte leaflets (see Figure $24 \mathrm{a} / \mathrm{e} / \mathrm{c}$ ). Instead of the uniformly filled cells of control stratum corneum, both mutant strains showed remnants of organelles (see Figure 24b/f and Figure 25b/c). Furthermore, the amount of these remnants was increased in Snap $29^{-/-}$epidermis compared to Snap $29^{\mathrm{fl} / \mathrm{fl}} / \mathrm{K} 14-$ Cre stratum corneum (see Figure 25).

The amount of secreted LB content between stratum granulosum and stratum corneum was strongly reduced in both mutants (see Figure 24c/h/i) compared to control epidermis (see Figure 24a/g). In control epidermis normal well-formed LBs were detectable (see Figure 24g), whereas Snap $29^{\mathrm{fl} / \mathrm{fl}} / \mathrm{K} 14-$ Cre and Snap $29^{-/-}$epidermis displayed electron lucent vesicular-like structures, presumably representing malformed LBs (see Figure 24h/i). However, normal shaped LBs were also present in the epidermis of both mutant mouse lines. Further structural examination using electron microscopy revealed numerous electron lucent structures in Snap $29^{-/-}$stratum corneum, presumably representing lipid droplets. These structures were also present in Snap $29^{\mathrm{fl} / \mathrm{fl}} / \mathrm{K} 14-$ Cre epidermis, but less frequent compared to the total knockout (see Figure 24b/c/f and Figure 25) (see Chapter 4.9). Furthermore, remnants of nuclei (parakeratosis) were found in the stratum granulosum and stratum corneum of Snap $29^{-1-}$ mice, but not in the epidermis of Snap29 $9^{\mathrm{fl} / \mathrm{fl}} / \mathrm{K} 14-\mathrm{Cre}$ mice (see Chapter 4.4).

In conclusion, formation as well as maturation of LBs is altered in Snap29-deficient epidermis. Furthermore, electron microscopic analysis of both mouse lines revealed some differences regarding LB secretion and organelle remnants (see Figure 25). 

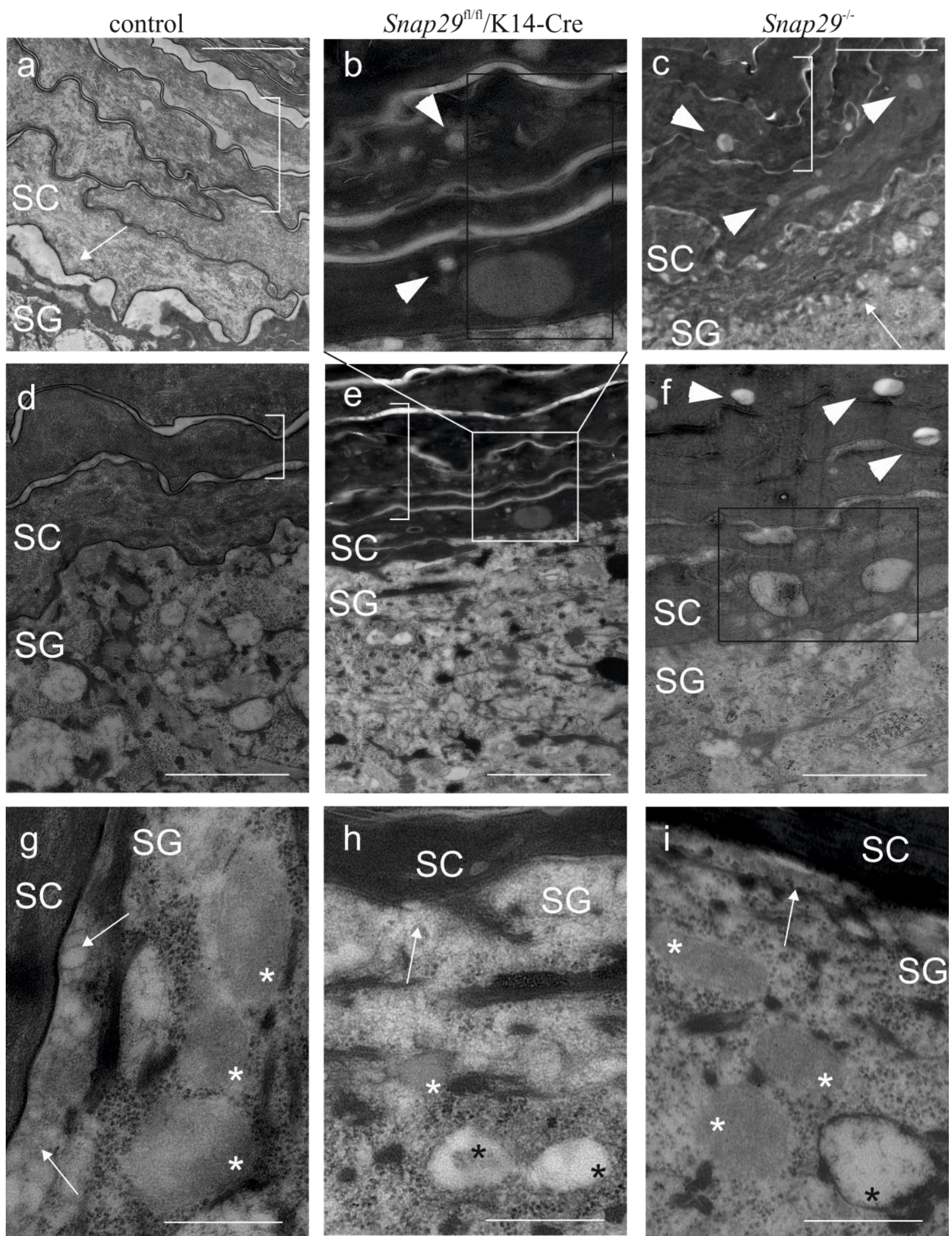

Figure 24: Ultrastructural analysis of control (a/d/g), Snap2 $9^{\mathrm{fl} / \mathrm{fl}} / \mathrm{K} 14-\mathrm{Cre}(\mathrm{b} / \mathrm{e} / \mathrm{h})$, and

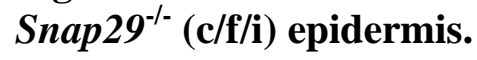

White arrows indicate secreted material between stratum granulosum and stratum corneum. White asterisks indicate LBs without pathological findings; black asterisks indicate LBs without structured content. White arrowheads indicate electron lucent structures, presumably indicating lipid droplets. Brackets show regions of fluffy stratum corneum structures in control skin and corresponding regions in mutant epidermis. Black squares indicate regions containing remnants of organelles. Panel (b) shows a higher magnification view of the marked area in panel (e). Scale bars: $2500 \mathrm{~nm}(\mathrm{a} / \mathrm{c}), 2000 \mathrm{~nm}(\mathrm{~d} / \mathrm{e} / \mathrm{f}), 500 \mathrm{~nm}(\mathrm{~g} / \mathrm{h} / \mathrm{i})$. SC: stratum corneum, SG: stratum granulosum. (Schiller, Seebode et al, 2015). 
Snap29-

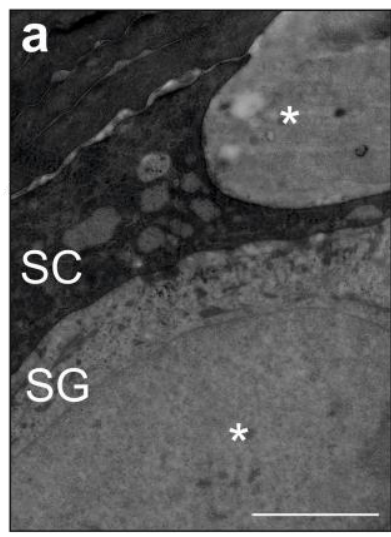

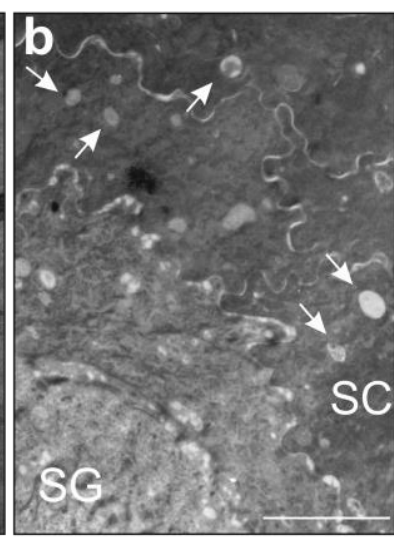

$\operatorname{Snap} 29^{\mathrm{fl} / \mathrm{fl}} / \mathrm{K} 14-\mathrm{Cre}$

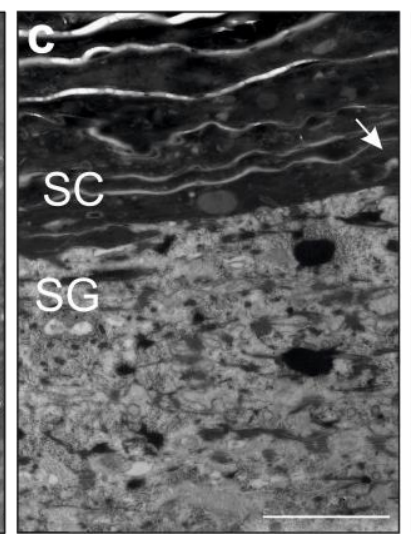

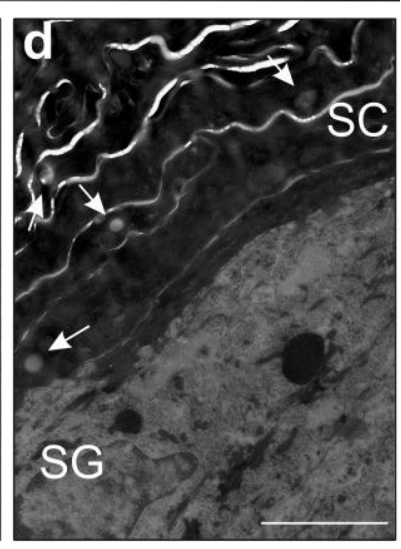

Figure 25: Ultrastructural variations between total and epidermal knockout mice.

Ultrastructural analysis of Snap29-/- (a/b), and Snap29 $9^{\mathrm{fl} / \mathrm{fl}} / \mathrm{K} 14-\mathrm{Cre}$ (c/d) mice. White asterisks indicate remnants of nuclei in the stratum granulosum and stratum corneum (parakeratosis). White arrows indicate electron lucent vesicle-like structures (b/c/d). Scale bars: 2000nm, SC: stratum corneum, SG: stratum granulosum.

\subsection{Snap29-deficient keratinocytes exhibit disturbed secretion of LB contents.}

Due to the finding of an altered formation and maturation of LBs in Snap29-deficient epidermis, the secretion of LB contents was examined by immunohistochemistry. In order to evaluate the potential consequences of Snap29 deletion on the formation of LBs and the secretion of their cargoes into the extracellular space, epidermal levels of kallikrein 7 (KLK7) and corneodesmosin (CDSN) were examined by immunohistochemistry (see Chapter 3.4.8.3). In general, desquamation relies on the controlled proteolytic degradation of corneodesmosomes by LB-derived proteases, such as kallikreins (reviewed by (IshidaYamamoto \& Kishibe, 2011)) (see Chapter 1.2.5).

KLK7 is a kallikrein-related peptidase involved in the cleavage of CDSN, which is secreted by LBs. In mutant epidermis the expression of KLK7 was strongly reduced compared to control skin (see Figure 26). In line with this finding, pathological remnants of CDSN were found in the stratum corneum of mutant mice, also reflecting a disturbed secretion of KLK7 (see Figure 26). During maturation of corneocytes, the sequential proteolytic cleavage of CDSN is a major prerequisite for desquamation (Jonca et al, 2011; Lundstrom et al, 1994).

In conclusion, the release of KLK7 into the extracellular space between stratum granulosum and stratum corneum is impaired, resulting in reduced levels of CDSN degradation and therefore in reduced desquamation of corneocytes in mutant epidermis. 

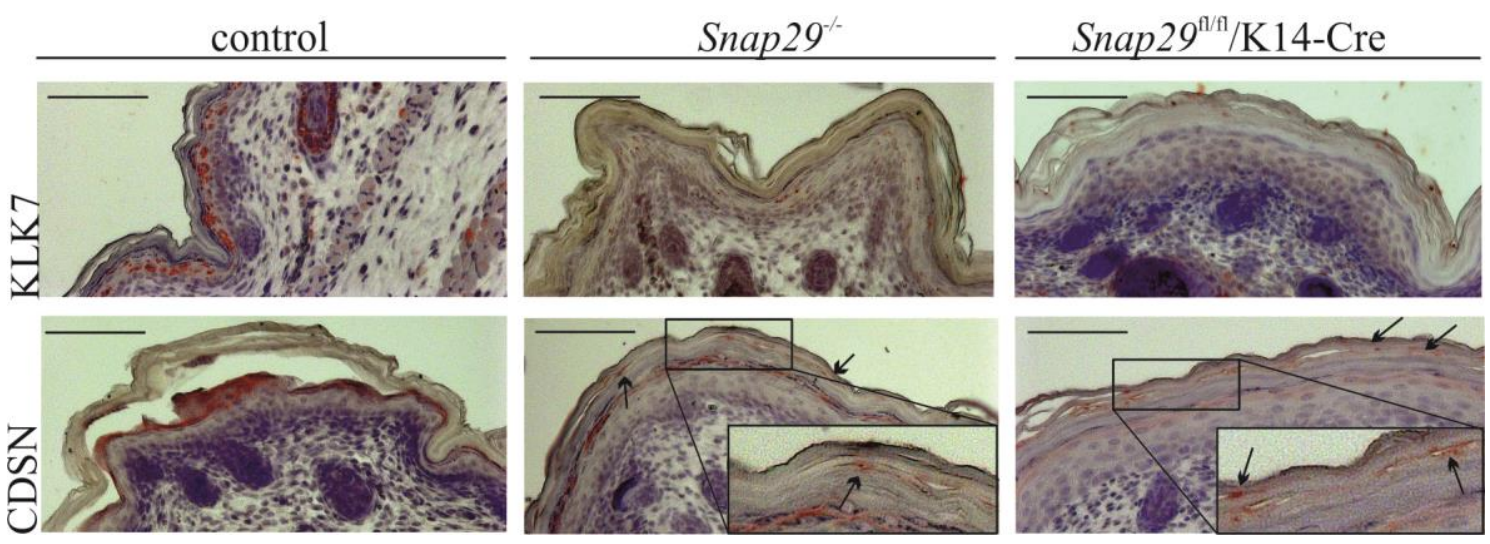

Figure 26: Disturbed desquamation in Snap29-deficient mice.

Immunohistochemical stainings for KLK7 and CDSN (see Table 7) showed decreased levels of both markers in Snap29-deficient epidermis. Remnants of CDSN were found in the stratum corneum of mutant mouse epidermis (black arrows). Panels below represent higher magnification views of the marked areas in the upper panel. Scale bars: $100 \mu \mathrm{m}$. (Schiller, Seebode et al, 2015).

\subsection{Snap29-deficient newborns show a defective epidermal lipid distribution.}

LBs transport glucosylceramides (GlcCer) and other lipids to the upper granular layers, which are essential for epidermal barrier formation (Madison et al, 1998). Since we observed a disturbed formation and maturation of LBs (see Chapter 4.7), epidermal lipid distribution was examined by BODIPY and Nile Red staining (see Chapter 3.4.8.5 and 3.4.8.6). In addition, indirect immunofluorescence with an antibody against glucosylceramide (GlcCer) was performed, which is a main lipid component in LBs and it is part of the intercellular lipid matrix.

Staining with the lipophilic dye Nile Red displayed a decreased deposition of neutral lipids in mutant mice epidermis (see Figure 27). Furthermore, indirect immunofluorescence staining (see Chapter 3.4.8.4) revealed a considerably reduced distribution of GlcCer in the cornified layers of Snap29-deficient mice, compared with the marked labeling in control epidermis (see Figure 27).

Ultrastructural analyses of Snap29-deficient epidermis showed numerous electron-lucent structures in the stratum corneum (see chapter 4.7). By BODIPY staining it was confirmed that these structures within the stratum corneum of Snap29-deficient mice are lipid droplets. In contrast to control epidermis, the stratum corneum of Snap29 $9^{\mathrm{fl} / \mathrm{fl}} / \mathrm{K} 14-\mathrm{Cre}$ newborns 
contained a multitude of lipid droplets (see Figure 27). These specialized intracellular organelles are found in many cell types, depositing neutral lipids, including triacylglycerols and sterol esters (Schmuth, 2003).

In summary, it can be concluded that Snap29-deficient newborns exhibit a defective epidermal lipid distribution.

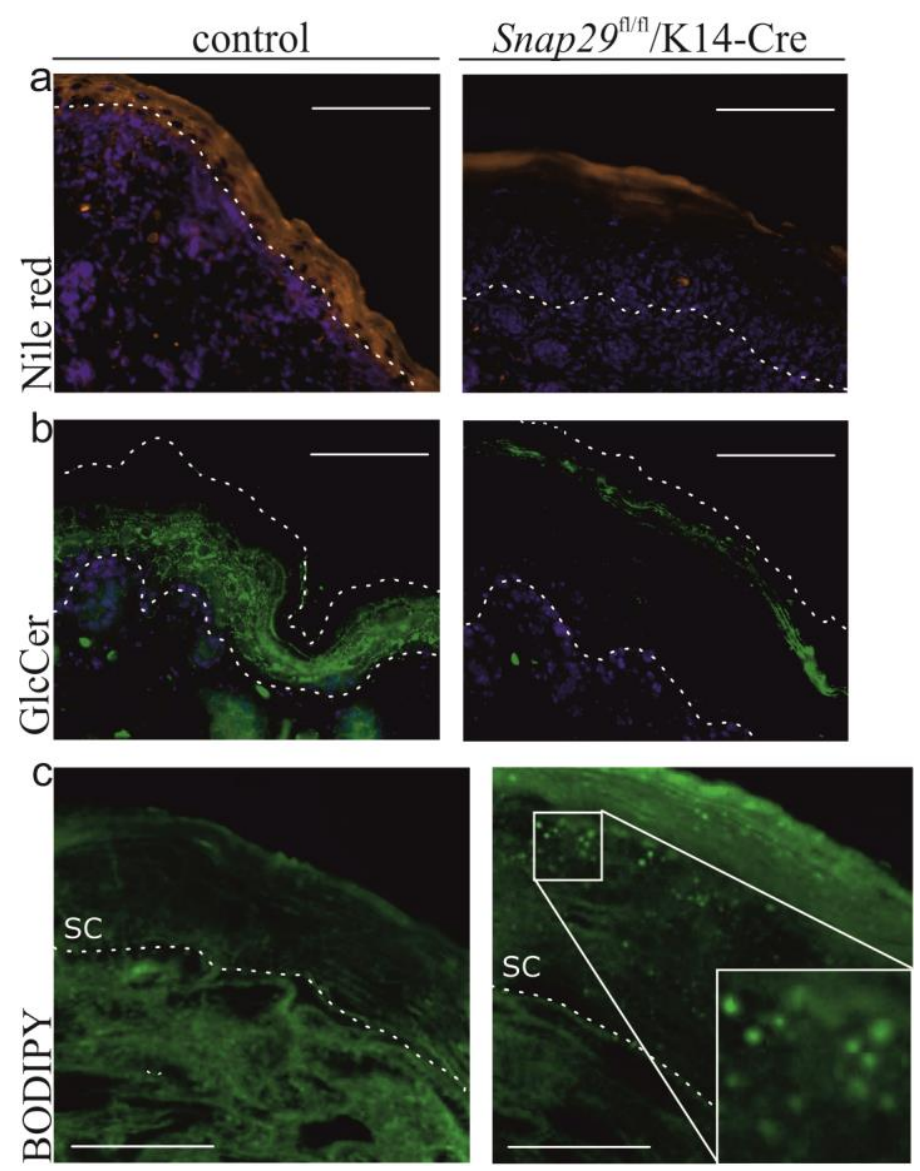

Figure 27: Defective epidermal lipid distribution in Snap29-deficient skin.

(a) Nile red staining showed a sparse deposition of neutral lipids in Snap29 $9^{\mathrm{fl} / \mathrm{fl}} / \mathrm{K} 14-\mathrm{Cre}$ stratum corneum. Scale bars: $100 \mu \mathrm{m}$. (b) Immunofluorescence staining using paraffinembedded skin sections revealed a decreased distribution of GlcCer the intercellular spaces of the cornified layers of Snap29 $9^{\mathrm{fl} / \mathrm{fl}} / \mathrm{K} 14-\mathrm{Cre}$ mice. Scale bars: $100 \mu \mathrm{m}$. (c) BODIPY 493/503 staining showed lipid droplets in Snap $29^{\mathrm{fl} / \mathrm{fl}} / \mathrm{K} 14-C r e$ stratum corneum, but not in control epidermis. Panel below represents higher magnification view of the marked area in the upper panel. Scale bars: $20 \mu \mathrm{m}, \mathrm{SC}$ : stratum corneum (Schiller, Seebode et al, 2015).

\subsection{SNAP29 deficiency causes impaired barrier permeability.}

Since epidermal lipids play a crucial role in the formation of a protective barrier against water loss and Snap29-deficient newborns exhibited a defective epidermal lipid distribution, the functional integrity of Snap $29^{\mathrm{fl} / \mathrm{fl}} / \mathrm{K} 14-\mathrm{Cre}$ epidermis was examined. Therefore, the fluid loss 
through the epidermis was determined, using a dehydration assay (outwards barrier function) and a dye intrusion assay (inwards barrier function) (see Chapter 3.4.8.7).

The loss of weight during a distinct period of time corresponds to the evaporation of water through the skin. Tested Snap29 $9^{\mathrm{fl} / \mathrm{fl}} / \mathrm{K} 14-\mathrm{Cre}$ mice showed a rapid and steady weight loss, whereas control littermates maintained their weight (see Figure 28). Therefore, the rapid weight loss observed in Snap $29^{\mathrm{fl} / \mathrm{fl}} / \mathrm{K} 14-\mathrm{Cre}$ mice may be attributed to the evaporation of water through the epidermis. During the experiment, mutant 2 urinated and consequently lost more weight in comparison to mutant 1 .

Additionally, the newborn mice were dyed with toluidine blue. The permeability of the skin for this dye indicates defects of the epidermal barrier. Snap $29^{\mathrm{fl} / \mathrm{fl}} / \mathrm{K} 14-\mathrm{Cre}$ newborns showed a dye intrusion across the entire epidermal surface, whereas control littermates were completely resistant to toluidine blue penetration (see Figure 28), indicating severe inside-out barrier defects in mutant mice epidermis.

In conclusion, the severe impairment of both inwards and outwards barrier functions of the epidermis in knockout animals highlights the pivotal role of SNAP29 in epidermal barrier function.
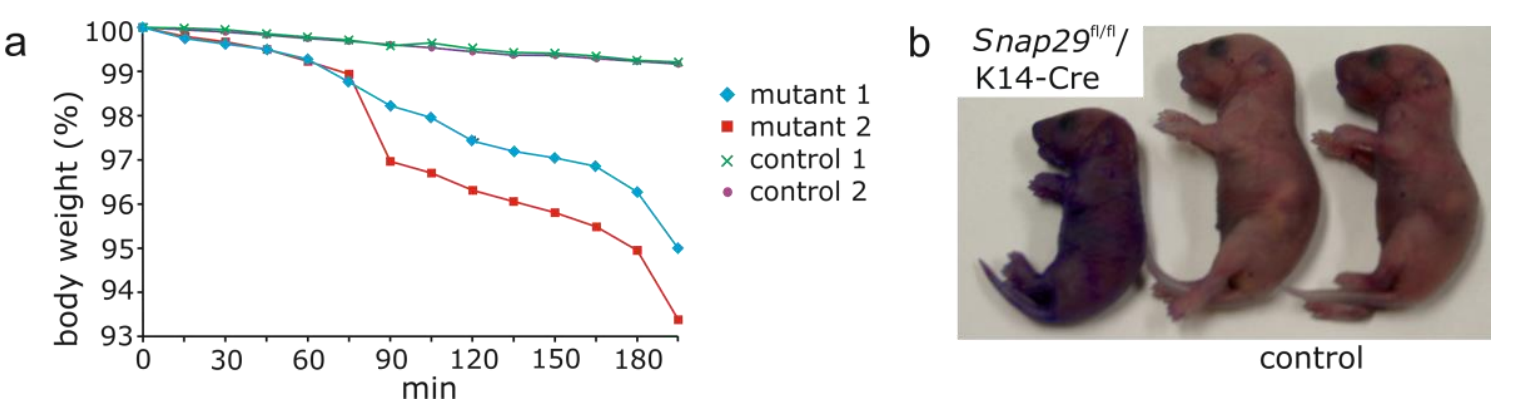

Figure 28: Epidermal barrier perturbation in Snap29 $9^{\mathrm{fl} / \mathrm{fl}} / \mathrm{K} 14-\mathrm{Cre}$ mice.

(a) Dehydration assay over time: Percentage of initial body weight of Snap29 $9^{\mathrm{fl} / \mathrm{fl}} / \mathrm{K} 14-\mathrm{Cre}$ mice and control littermates. The weight of four newborn Snap29 $9^{\mathrm{fl} / \mathrm{fl}} / \mathrm{K} 14-\mathrm{Cre}$ mice was measured every $15 \mathrm{~min}$ for 3 hours using a precision scale. Snap $29^{\mathrm{fl} / \mathrm{fl}} / \mathrm{K} 14-\mathrm{Cre}$ mice lost approximately $5 \%$ of their body weight within three hours, whereas control littermates maintained their weight. Note that mutant 2 urinated during the measurements and died after 3 hours. (b) Dye-penetration assay: Penetration of toluidine blue into the skin of Snap $29^{\mathrm{fl} / \mathrm{fl}} / \mathrm{K} 14-\mathrm{Cre}$ and control mice. Control epidermis was impermeable for the dye and control animals did not show any skin staining. In contrast, toluidine blue permeated the epidermal barrier of Snap29 $9^{\mathrm{fl} / \mathrm{fl}} / \mathrm{K} 14-\mathrm{Cre}$ mice (Schiller, Seebode et al, 2015). 


\subsection{Snap29 deficiency causes ER stress}

The formation of lipid droplets (see Chapter 4.9) as seen in Snap29-deficient epidermis (see Figure 27) can be triggered by endoplasmic reticulum (ER) stress. A major function of the ER is the coordination of protein folding as well as trafficking, and the majority of lipids are synthesized at the ER site. Concordantly, disturbances in ER function and structure lead to ER stress. In order to overcome ER stress, a specific ER quality-control system, called the unfolded protein response (UPR), is activated. However, in the presence of prolonged stress and severely impaired ER function, disturbances in ER homeostasis lead to apoptosis (reviewed by (Kadowaki \& Nishitoh, 2013)). In order to restore ER homeostasis, the UPR can promote a cellular repair process, including the activation of several proteins. In this study, UPR activation was assessed by investigating the protein level of CHOP (C/EBP homologous protein), whose induction has been reported to occur during ER stress (Samali et al, 2010). CHOP, also known as growth arrest and DNA damage-inducible gene 153 (GADD153), is a member of the C/EBP transcription factor family (Anand et al, 2005) and it was used as a marker for UPR activation.

In order to determine whether Snap29 deficiency affects additional ER stress, epidermal levels of CHOP were examined by immunohistochemistry and western blot analysis (see Chapter 3.4.8.3 and 3.5.4). In Snap29 $9^{\mathrm{fl} / \mathrm{fl}} / \mathrm{K} 14-\mathrm{Cre}$ as well as in control epidermis CHOP was detected (see Figure 29a). In order to ascertain whether a significant increase in CHOP expression is found in the skin of mutant mice, western blot analyses were performed with fibroblasts derived from total Snap29 knockouts. Evidently more CHOP was detected in fibroblasts derived from mutant mice compared to control fibroblasts (see Figure 29b/c).

In summary, it could be envisaged that Snap29 deficiency alters normal ER homeostasis and leads to UPR activation. 

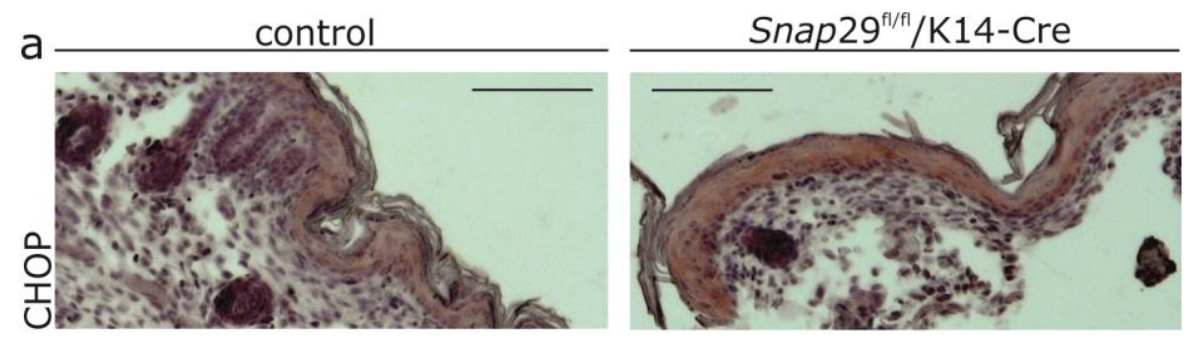

b

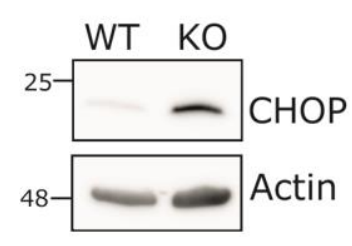

C

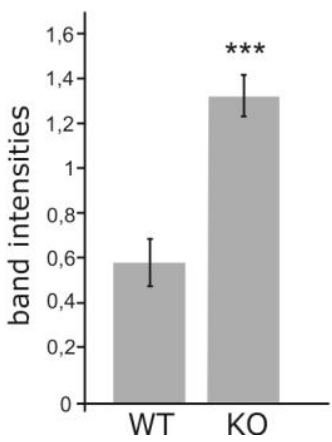

Figure 29: Detection of protein levels of CHOP.

(a) Immunohistochemical detection of CHOP in skin samples. Scale bars: $100 \mu \mathrm{m}$. (b) Western Blot analyses were performed with $25 \mu \mathrm{g}$ whole cell lysate (MEFs), using a specific antibody against endogenous CHOP. As loading control $\beta$-actin was used (see Table 7). Representative western blot shows a significant upregulation of CHOP. (c) The western blot band intensities were measured with ImageJ software. The graph shows the mean of three independent experiments and their respective standard deviation. For calculation of statistical significances the student's t-test was used $(* * *=p<0.001)$.

\subsection{Snap29 deficiency alters autophagy}

Recent studies revealed an involvement of SNAP29 in autophagy through the direct control of the fusion of the autophagosomal membrane with the lysososomal membrane (see Chapter 1.6.2) and it was hypothesized that ER stress is a potent inducer of autophagy (Ogata et al, 2006). Furthermore, our data clearly indicate a delay in the degradation of organelles in the stratum corneum (see Chapter 4.7). Keratinocyte differentiation involves organelle clearance and recycling of cellular components and autophagy is an important element involved in this process (Belleudi et al, 2014; Moriyama et al, 2014). The increasing significance attached to SNAP29 in autophagy has generated the need to examine autophagic activation in Snap29deficient cells.

In order to determine whether Snap29 deficiency affects autophagy, levels of LC3B-II as well as p62/SQSTM1 were analyzed by western blot under normal and starvation conditions (see Chapter 44). The membrane-associated LC3B-II is derived by conversion of its cytosolic form (LC3B-I) and can be used as a well-established marker for autophagosomes (see 
Chapter 1.6.1). Western blot analyses were performed using protein extracts from Snap29deficient fibroblasts obtained from Snap $29^{-/-}$and control mice. Immunoblot analyses revealed markedly increased LC3B-II protein levels in Snap29-- cells under basal as well as under starvation conditions (see Figure 30a/c).

Given that autophagy is a highly dynamic and complex process, the accumulation of autophagosomes in mutant cells could indicate either an induction of autophagy or a block in autophagic flux (see Chapter 1.6.1). Therefore, the mere detection of LC3 turnover is insufficient for an overall evaluation of this process. In order to distinguish between these possibilities, levels of p62, also known as SQSTM1/sequestosome 1, were analyzed (see Chapter 1.6.1) (Bjorkoy et al, 2005). p62 protein amounts remained largely unchanged in Snap $29^{-/-}$cells under basal as well as starvation conditions (see Figure 30b).

a

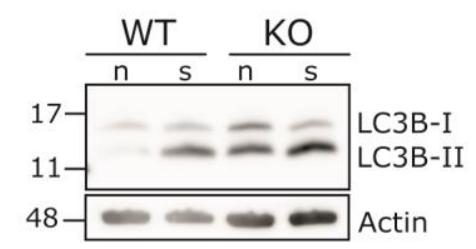

C

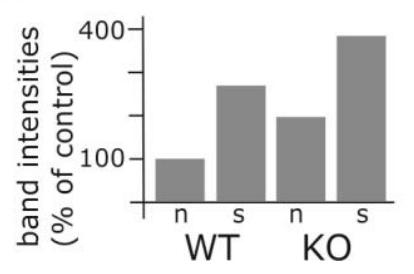

b

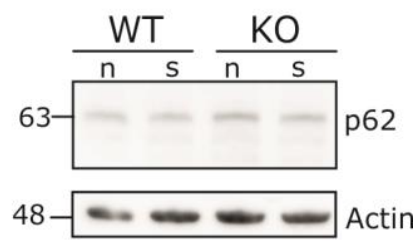

Figure 30: Snap29 deficiency affects autophagy.

(a) Representative western blot for LC3B-I and -II levels in control and Snap29-deficient primary fibroblasts cultured under normal (n) and starvation conditions (s). For starvation conditions, fibroblasts were cultured for $2 \mathrm{~h}$ in EBSS. (b) Levels of p62/SQSTM1 in control and Snap29-deficient primary fibroblasts cultured under normal (n) and starvation conditions (s). Western Blot analyses were performed with $25 \mu \mathrm{g}$ whole cell lysates, using a specific antibody against endogenous LC3B and p62/SQSTM1 (see Table 7). $\beta$-actin was used as loading control. The blots are representative for at least two independent experiments showing similar results. (c) The bar graph shows the ratio of LC3B-II against $\beta$-actin. The relative marker protein expression of control cells under normal conditions was set to $100 \%$. The western blot band intensities were measured with ImageJ software (Schiller, Seebode et al, 2015). 
In order to accurately assess autophagy, autophagic flux was further assessed by LC3-II turnover using western blot analysis (see Chapter 3.5.4) in the presence and absence of bafilomycin $\mathrm{A} 1$, an inhibitor for the vacuolar type $\mathrm{H}^{+}$-ATPase reliant for lysosomal acidification (see Chapter 1.6.1). If autophagic flux is occurring, the degradation of LC3-II through the autophagic pathway is blocked in the presence of the inhibitor, resulting in accumulation of LC3B-II (Tanida et al, 2005). LC3B-II levels were increased under EBSS treatment (see Figure 30a) and were further increased by the combination of EBSS and bafilomycin A1 in Snap29 knockout cells and control cells (see Figure 31), which suggests that effects of Snap29 deficiency on autophagy were based on an enhanced autophagic flux.

In summary, our experiments point towards an induction of autophagy, because no strikingly enhanced levels of p62 were detected and the levels of LC3B-II increased in the presence of bafilomycin A1, especially in mutant cells.

a

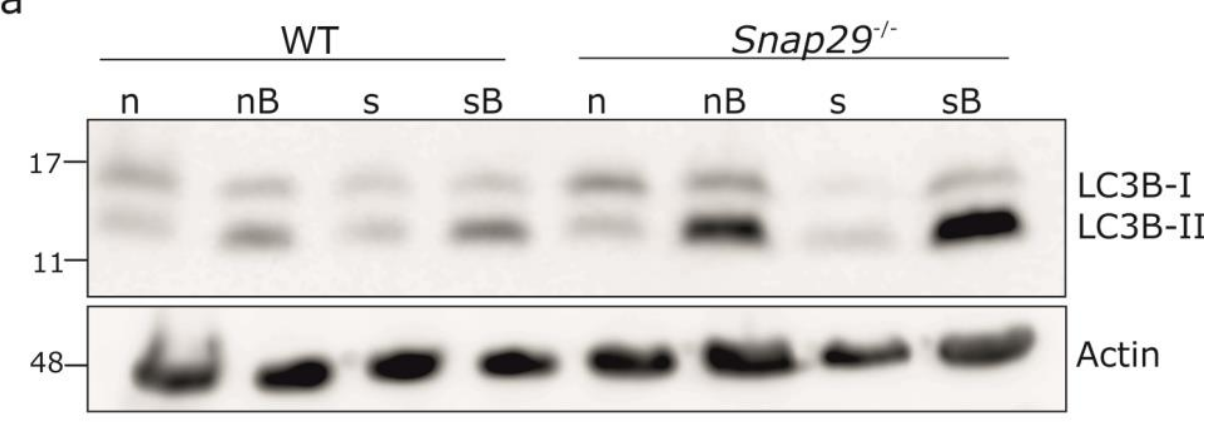

b

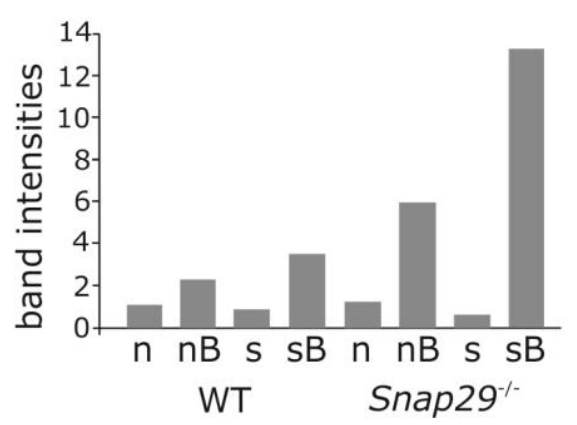

Figure 31: Effects of bafilomycin A1 on LC3B-II levels.

Representative western blot for LC3B-I and -II levels in control and Snap29-deficient primary fibroblasts cultured under normal (n), starvation conditions (s) and under treatment with bafilomycin A1 (B). Primary fibroblasts were grown in normal medium (n), treated for $2 \mathrm{~h}$ with 100nM bafilomycin A1 in normal medium (nB), or grown in EBSS in the absence (s) or presence of $100 \mathrm{nM}$ bafilomycin A1 (sB). Western Blot analysis was performed with $25 \mu \mathrm{g}$ whole cell lysates, using a specific antibody against endogenous LC3B. $\beta$-actin was used as loading control. The numbers left to the blots indicate protein size in $\mathrm{kDa}$. (b) The bar graph shows the ratio of LC3B-II against $\beta$-actin. The relative marker protein expression of control cells under normal conditions was set to $100 \%$. The western blot band intensities were measured with ImageJ software. 


\section{Discussion}

Epidermal differentiation requires membrane fusion between vesicles and target membranes for intracellular membrane trafficking for what SNARE proteins have been recognized as the core protein machinery (reviewed by (Jahn \& Scheller, 2006)). An important recent development has been the discovery that homozygous loss-of-function mutations in the SNAP29 gene, encoding a member of the SNAP family, cause the human CEDNIK syndrome (see Chapter 1.3). Examining the molecular basis of this neurocutaneous syndrome revealed the pivotal role of SNAP29 in epidermal differentiation (Rapaport et al, 2010; Sprecher et al, 2005).

However, despite the presence of cutaneous abnormalities, little is known about the consequence of a defective SNAP29 function during terminal differentiation of keratinocytes. Therefore, the aim of my thesis was to create Snap29-deficient mouse lines to further investigate the role of SNAP29 in epidermal differentiation, especially on LB formation and maturation. This experimental approach contributes to a further disclosure of the pathogenesis of the human CEDNIK syndrome.

\subsection{Generation of two Snap29-deficient mouse models}

Due to the very limited number of CEDNIK syndrome patients (see Chapter 1.3), a mouse model is needed to facilitate the study of SNAP29 in epidermal differentiation. Furthermore, mouse models are most commonly used for investigating human disease. So far, three Snap29-deficient non-mammalian animal models have been described (Caenorhabditis elegans (Guo et al, 2014; Kang et al, 2011; Sato et al, 2011), Danio rerio (Li et al, 2011), and Drosophila melanogaster (Xu et al, 2014)). Nonetheless, these models are not sufficient to elaborate the role of SNAP29 in epidermal differentiation, because non-mammalian epidermis does not undergo cornification, which in mammals leads to the development of the stratum corneum and the associated barrier function of the epidermis (see Chapter 1.2). Until today, no mouse mutants for Snap29 have been described and it was suggested that Snap29 knockout mice are embryonic lethal ( $\mathrm{Lu}$ et al, 2015). However, in this present study I report the successful generation and characterization of a total knockout as well as a keratinocytespecific conditional Snap29-deficient mouse line as models for the very rare human CEDNIK syndrome (Schiller, Seebode et al, 2015). 


\subsection{Snap29-deficient mice mimic the human CEDNIK syndrome phenotype}

Ichthyoses are genetically determined Mendelian disorders of cornification (MEDOC) that can be distinguished between non-syndromic and syndromic forms (Traupe et al, 2014). CEDNIK syndrome is an example of syndromic ichthyoses, in which the cutaneous manifestations are accompanied by clinical symptoms within a number of other organ systems. The epidermal phenotype of CEDNIK syndrome patients is characterized by palmoplantar keratoderma and ichthyosis (see Chapter 1.3 and Figure 4) (Sprecher et al, 2005). Both Snap29-deficient mouse lines showed a distinct ichthyosiform phenotype (see Chapter 4.3), which closely reproduces the ichthyosis phenotype seen in human patients with SNAP29 mutations. Furthermore, we observed many CEDNIK features in our mutant mice, including hyperkeratosis, impaired LB secretion and maturation, alterations in epidermal lipid distribution and levels, and an impaired skin barrier function. Thus, both Snap29 mutant mouse lines provide a mammalian model for the human CEDNIK syndrome (Schiller, Seebode et al, 2015).

Nonetheless, the murine skin phenotype is more severe compared to the cutaneous alterations in human patients. Epidermal as well as total Snap29-deficient mice displayed a congenital ichthyosis with constrictive and taut skin as well as ichthyotic lesions, resulting in early lethality within a few hours after birth (see Chapter 4.3). However, the human epidermal phenotype is often characterized by a rather mild and late-onset ichthyosis with symptoms developing early in childhood (between 5 and 11 months of age) (Sprecher et al, 2005) (see Chapter 1.3). More recently, however, another case of two siblings suffering from CEDNIK syndrome has been mentioned. Besides the common features described for this syndrome, the affected children showed phenotypic variations, including a congenital generalized ichthyosis with a collodion membrane at birth. It was hypothesized that this variability could be due to the influence of modifier genes (Ben-Salem et al, 2015). Furthermore, CEDNIK syndrome patients show solely hyperkeratosis (Fuchs-Telem et al, 2011; Sprecher et al, 2005), but no additional ichthyotic features like acanthosis as seen in Snap29-deficient mice (see Chapter 4.4).

The observed differences regarding the ichthyosiform phenotype between the murine model and the human disease might result from different stages of skin development at birth in mice and humans (Eckl et al, 2013). Concordantly, the development of more pronounced phenotypes accompanied by neonatal lethality is commonly observed in murine models of 
human ichthyosiform diseases (Epp et al, 2007; Radner et al, 2010). Furthermore, gene defects resulting in impaired barrier function often cause a more severe phenotype in the murine than in human skin as a result of a less advantageous body surface to volume ratio in small rodents (de Juanes et al, 2009).

Taken together, SNAP29 is implicated in the development of the skin phenotype in both the human patients and in the Snap29-deficient mouse models. Unfortunately, the early lethality within a few hours after birth prevented a further characterization of the epidermal phenotype in adult mice. A suitable approach in order to overcome early postnatal lethality might be a tamoxifen-inducible Cre-lox recombination technology.

\subsection{SNAP29 is essential for a functional epidermal barrier.}

Notably, Snap29-deficient mice revealed a severely impaired skin permeability function (see Figure 28) and the fact that both mutant mouse lines died shortly after birth (see Chapter 4.3), suggests that the neonatal mice died because of their severe cutaneous phenotype. Most mouse models with epidermal barrier defects suffer from postnatal lethality, often as a result of severe transepidermal water loss. Snap29-deficient mice lost approximately 5\% of their body weight within the first three hours after birth, and other knockout mouse models with epidermal permeability barrier defects, e.g. mice deficient for LEKTI (Descargues et al, 2005), exhibited comparable weight loss (Epp et al, 2007), whereas these transgenic mice showed a longer lifespan compared to our mice. Furthermore, 12R-LOX-deficient mice, which harbor loss-of function mutations in the Alox $12 b$ gene, lost for example $10 \%$ of the bodyweight within one hour as a result of severe barrier perturbations and died three to five hours after birth (Epp et al, 2007). By comparison, Snap29-deficient mice lost substantially less water and it is likely that the transepidermal water loss is not the sole cause for neonatal lethality in our mice.

Additionally, Snap29-deficient animals presented a restrictive movement capability and no suckling behavior was observed (see Chapter 4.3). Interestingly, the K14 promoter targets Cre recombination not exclusively to the epidermis, but also to tongue and esophagus (Qiu et al, 2011) and EIIa gene products are ubiquitously expressed (see Chapter 4.1). Thus, both promoters are active in esophagus and oral epithelium, having likely an additional effect on early lethality due to suckling difficulties. Therefore, the severe skin phenotype due to a severely impaired inward and outward permeability barrier function, accompanied by 
impaired dietary intake most likely leads to the early postnatal death of Snap29-deficient animals.

\subsection{Snap29 deficiency provokes a disturbed LB appearance}

SNAP29 deficiency is known to result in impaired maturation and secretion of LBs, particularly interfering with the transport and exocytosis of lipids from granular cells into the extracellular spaces of the stratum corneum. The most striking ultrastructural feature in the epidermis of human CEDNIK patients is the presence of vesicles with various sizes and contents (Sprecher et al, 2005)(see Chapter 1.3). CEDNIK patients' epidermis displayed numerous electron-lucent vesicles in the upper epidermal layers and additionally in the in the lower stratum corneum. It was hypothesized that these vesicles represent empty or malformed LBs. It is noteworthy, that normal-shaped LBs were also found in the epidermis of CEDNIK patients (Sprecher et al, 2005). Currently, it remains unclear to which extent this electronlucent vesicles possess normal LB function in the epidermis of CEDNIK syndrome patients. Consistent with these data, comparable ultrastructural alterations regarding LB morphology were obtained in Snap29-deficient mouse epidermis. LBs with normal as well as electronlucent appearances lacking the typical lamellar structures were present in the stratum granulosum. Furthermore, electron-lucent vesicle-like structures were also observed in the stratum corneum (see Chapter 4.7). These observations closely resemble findings in human patients, and additionally in a Snap29-deficient Danio rerio model, which also displayed electron-lucent vesicles in the epidermis ( $\mathrm{Li}$ et al, 2011).

Other cutaneous diseases which are characterized by disturbances in intracellular organelle trafficking are, for example, ARC syndrome and Harlequin ichthyosis. The identification of diseases affecting LB function is a crucial advance for understanding the molecular basis of LB function. ARC syndrome is caused by a mutation in the SM (Sec1/Munc18) protein VPS33B (vacuolar protein sorting 33 homolog B), which is involved in the regulation of vesicular membrane fusion by interacting with SNARE proteins (Gissen et al, 2006). Ultrastructural examinations of patient's skin samples revealed retention of normal appearing LBs in corneocytes (Hershkovitz et al, 2008). Despite the fact that impaired vesicle fusion leads to an impaired LB secretion in both ARC and CEDNIK syndrome (Gissen et al, 2006; Steegmaier et al, 1998), LB maturation was markedly disrupted in CEDNIK syndrome (Sprecher et al, 2005), whereas the internal structure of LBs appeared normal in ARC syndrome (Hershkovitz et al, 2008; Zhou \& Zhang, 2014). These data are indicative for a role 
of SNAP29 controlling key steps in exocytosis as well as in the formation/maturation of LBs, whereas VPS33B is exclusively involved in LB secretion.

Loss-of-function mutations in the $A B C A 12$ gene, encoding for the ATP-binding cassette transporter A12 - a lipid transporter in LBs - have been reported to cause Harlequin ichthyosis. This cutaneous disease is likewise characterized by disturbances in the transport of lipids from the Golgi to LBs. In Harlequin ichthyosis abnormally shaped, reduced, or absent LBs were found in the epidermis (Scott et al, 2013). As a result, defective intracellular trafficking especially interfering with lipid transport can result in phenotypically and ultrastruturally similar alterations in the epidermis.

However, it should be noted that perturbations in LB morphology were also observed in human filaggrin knockdown organotypic cultures, which showed LBs varying in size and lacking their typical lamellar structure. The filaggrin-deficient skin model revealed barrier perturbations which were not accompanied by altered lipid composition (Mildner et al, 2010). These findings suggest the presence of additional indirect factors which might orchestrate LB formation. As a result, it remains to be elucidated whether SNAP29 is directly or indirectly involved in this process.

\subsection{Snap29 deficiency alters the epidermal lipid structure}

As transport units for barrier components, LBs serve as key factors for the composition, formation, and maintenance of the epidermal barrier (Elias et al, 1998; Ishida-Yamamoto et al, 2004; Norlen, 2001; Raymond et al, 2008)(see Chapter 1.2.4). Due to the finding of malformed or empty LBs in Snap29-deficient mice (see Chapter 4.7), we assumed that the observed perturbations in barrier function might be caused by a defective formation of lipid lamellae. This lamellar structure within the cornified layers is essential for the formation and homeostasis of an intact epidermal permeability barrier (Wertz, 2000). Under physiological conditions LBs deliver and secret precursor lipids (GlcCers) to the transition area which fill up the intercellular space between the corneocytes (Ishida-Yamamoto et al, 2004) (see Chapter 1.2.3). GlcCers are the main constituent of LBs and they are converted into ceramides, which represent substantial structural components of intercellular lipid lamellae (Wertz, 2000). During histological preparations, these intercellular lipids get lost causing the characteristic fluffy, basket-weave like appearance of the stratum corneum in histologic sections. However, histological examinations as well as electron micrographs of the murine 
Snap29-deficient epidermis displayed a condensed stratum corneum with a reduced number of lacunas, indicating a diminished formation of the intercorneocyte lipid lamellae. Concordantly, ultrastructural analyses revealed decreased amounts of secreted LB content in the transition area between stratum granulosum and stratum corneum in our mouse models (see Chapter 4.7). Moreover, we detected reduced levels of GlcCer in the murine Snap29deficient epidermis (see Chapter 4.9). In good agreement with these findings, diminished amounts of GlcCer were also found in the epidermis of human CEDNIK syndrome patients (Sprecher et al, 2005).

Furthermore, it was shown that mice with an epidermis-specific glucosylceramide synthase deficiency displayed abnormalities in LB as well as in stratum corneum structure accompanied by an increased transepidermal water loss (Jennemann et al, 2007). In addition, a defective trafficking of GlcCer into LBs due to ABCA12-deficiency in decreased LB contents as well as decreased amounts of the extracellular lipid lamellae within the stratum corneum, leading to an impaired barrier function (Akiyama, 2011a; Dale et al, 1990; Milner et al, 1992). Thus, these data together with our observations are consistent with a substantial role of GlcCer in epidermal barrier homeostasis (Feingold, 2007).

Since LBs are hypothesized to be a part of the tubulo-vesicular TGN (Elias et al, 1998; Madison, 2003) and SNAP29 was found to localize to intracellular membranes such as the Golgi apparatus and endocytic vesicles (Rapaport et al, 2010; Wong et al, 1999), these data support the role of SNAP29 in mediating trafficking from the Golgi apparatus via lipidcontaining LBs to the intercellular space (Rapaport et al, 2010; Sprecher et al, 2005). Interestingly, it was suggested that ABCA12 is essential for lipid transport from the Golgi apparatus to LBs in keratinocytes (Sakai et al, 2007).

From these findings, we suggest that ablation of SNAP29 provokes perturbations in LB secretion of lipids to the interstices of corneocytes, leading to a defective formation of the intercellular lipid lamellar structure of the stratum corneum, which most likely results in severe barrier perturbations in Snap29-deficient mice. The clinical importance of this lamellar structure has been reported in several studies (reviewed by (Madison, 2003)) and many congenital ichthyoses, despite their genetic heterogeneity, are considered as disorders of a disturbed epidermal lipid metabolism associated with prominent barrier abnormalities (Elias et al, 2012). 
Consistent with disturbed GlcCer levels, a substantial reduction of neutral lipids in the neonatal knockout skin was additionally observed (see Chapter 4.9). Furthermore, numerous lipid droplets were detected in the stratum corneum of Snap29-deficient mice. Notably, the observed cutaneous phenotype of Snap29-deficient mice mostly resembled defects in epidermal barrier function, as seen in ABCA12-deficient mice which were characterized by hyperkeratosis, abnormal LB morphology and retention of lipid droplets (Smyth et al, 2008; Yanagi et al, 2008). In general, lipid droplets arise from the ER and store neutral lipids. Interestingly, accumulation of these specialized organelles has been described under induced ER stress (Hapala et al, 2011), after exposure of the epidermis to several irritants, e.g. retinoids, or in states of a disturbed epidermal lipid metabolism (Schmuth, 2003).

\subsection{Increased proliferation and abnormal keratinocyte differentiation in Snap29-deficient mice epidermis}

Snap29-deficient mice displayed hyperkeratosis and epidermal hyperplasia, which were associated with an increased number of proliferating cells in the basal layer of the epidermis as well as K6 expression (see Chapter 4.6). In humans, hyperproliferation represents a typical mechanism to compensate epidermal barrier defects as a repair process (Ny \& Egelrud, 2004) and similar morphological hyperproliferative conditions were observed in the epidermis of the human skin diseases like lamellar ichthyosis, atopic dermatitis, and psoriasis (Oji \& Traupe, 2006). Furthermore, it is also a common feature observed in the adult epidermis of other mouse models suffering from severe skin barrier defects (Segre, 2003). However, the hyperkeratosis of Snap29-deficient mice displayed directly after birth, being unlikely a direct compensatory response to the epidermal barrier defect. Furthermore, the expression of keratinocyte differentiation markers was dysregulated. Snap29-deficient keratinocytes lost their ability for normal terminal differentiation and expressed K14 throughout suprabasal layers (see Chapter 4.5). Hence, loss of Snap29 leads to aberrant terminal differentiation of basal keratinocytes.

Moreover, immunohistochemistry revealed a reduction of keratohyalin granules within the granular layer and a reduced expression of filaggrin in the stratum granulosum of Snap29deficient mice (see Chapter 4.5), which are commonly observed histomorphological features in the skin of patients with ichthyosis vulgaris (Smith et al, 2006; Sybert et al, 1985). Concordantly, the reduced level of this structural protein might also contribute to the formation of condensed stratum corneum, which might additionally be involved in the 
observed defects in barrier permeability. This is in concordance with data from other authors reporting a disturbed epidermal barrier due to a defective proteolytic processing of filaggrin into its monomers (Descargues et al, 2005; Leyvraz et al, 2005; List et al, 2002; Presland et al, 2000).

Immunohostochemical analyses revealed a diminished proteolytic level in the epidermis of Snap29-deficient mice, indicating that the hyperkeratosis in Snap29-deficient epidermis might be partly due to a reduced desquamation of corneocytes. Furthermore, remnants of CDSN were observed in the stratum corneum (see Chapter 4.8), which is normally devoid of this corneodesmosomal protein. Since LBs transport and secret several proteins, proteases, and protease inhibitors, which are essential for the generation and maintenance of the epidermal barrier (Ishida-Yamamoto et al, 2004) (see Chapter 1.2.4), it is likely that disturbances in LB function effect localization and levels of their cargoes such as CDSN, which undergoes proteolytic cleavage mainly by the specific serine proteinases KLK7 and KLK5 (Caubet et al, 2004). Concordantly, controlled proteolytic degradation of corneodesmosomes is essential for physiological desquamation. Our findings indicate a delayed corneodesmosomal degradation process as a result of reduced amounts of KLK7. This is consistent with previous studies indicating that abnormalities in transportation and secretion of LBs result in reduced desquamation (Ishida-Yamamoto \& Kishibe, 2011; Rassner et al, 1999). In good agreement with our findings, remnants of CDSN were also found in a human SNAP29 down-regulated organotypic keratinocyte cell culture model (Fuchs-Telem et al, 2011). Taken together, our results suggest that the observed hyperkeratosis is not a compensatory mechanism to counteract barrier perturbation, but represents more likely a retention hyperkeratosis due to a defective differentiation and desquamation processes. Additionally, this feature can be attributed to structural and functional alterations of LB function.

Furthermore, lipid precursors are essential for the incorporation of the hydrolytic enzymes into LBs (Rassner et al, 1999), and since we observed diminished amounts of GlcCer in Snap29-deficient mice epidermis, the defective lipid trafficking could also impair the transportation of KLK7 to LBs. This is in good agreement with the a recent line of published data, which suggests that a lipid transport defect impairs the delivery of proteases to LBs in the skin of Harlequin ichthyosis patients, resulting in hyperkeratosis (Elias et al, 2008).

In conclusion, Snap29 depletion leads to aberrant keratinocyte differentiation, a reduced serine protease level accompanied by delayed desquamation (see Figure 26) and a dysregulation of keratinocyte differentiation markers (see Figure 19). These features are 
presumably caused by general disturbances in vesicular trafficking, leading to the observed ichthyotic phenotype in Snap29-deficient mice.

\subsection{Impacts of Snap29 deficiency on ER function}

The observation of lipid droplets in the stratum corneum of Snap29-deficient mice (see Figure 27) prompted us to investigate ER function. The ER serves as the main site for the synthesis as well as homeostasis of lipids. As already stated, lipid droplets arise from the ER and theses dynamic structures often accumulate under various cellular stress conditions such as induced ER stress (Wilfling et al, 2014) (see Chapter 5.5). Besides being storage compartments for neutral lipids, they are formed in order degrade excessive amounts of lipids accumulated at the ER. Lipid droplets might also be important for the degradation of un- or misfolded proteins (Hapala et al, 2011).

The ER stress response was originally considered as a compensatory mechanism in order to overcome pathological conditions, but it has emerged that this reaction is also involved in physiological cellular differentiation. For example, studies revealed that CHOP was induced in the suprabasal layers of human epidermis during normal keratinocyte differentiation (Sugiura et al, 2009). Thus, keratinocytes are reliant upon a slight UPR activation for normal differentiation (Sugiura, 2013). In conjunction with this, immunohistochemical staining revealed CHOP staining in the epidermis of our mice. Furthermore, a marked increase of CHOP levels in Snap29-deficient fibroblasts compared to control cells was detected (see Chapter 4.11). Therefore, the accumulation of lipid droplets within corneocytes of Snap29deficient mice appeared to be caused by an increased ER stress response in order to prevent the accumulation of excessive lipids and unfolded proteins at the ER membrane. Notably, lipid droplets were also observed within corneocytes as ultrastructural feature of Congenital ichthyosis type 1 (Niemi et al, 1994), and additionally in other pathologies such as cardiomyopathies, neuropathies, or during viral hepatitis (Vallet-Pichard et al, 2012), which are not associated with defects in lipid metabolisms.

Furthermore, a recent study elucidated a novel link between barrier homeostasis of the epidermis and the response to ER stress. It was anticipated that $\mathrm{Ca}^{2+}$ release from the ER as a response to cellular stress conditions is associated with $\mathrm{LB}$ secretion. This $\mathrm{Ca}^{2+}$ release ensures that LB secretion takes place prior to cornification (Celli et al, 2011). Thus, it is an important signal for epidermal permeability barrier repair. Since Snap29 deficiency correlates 
with epidermal barrier permeability in our knockout mice, the ER stress response might be a signal for triggering LB secretion in order to overcome the defective barrier (see Chapter 1.2.4).

Together, these results clearly indicate evidence of the link between Snap29 deficiency and altered ER function. Furthermore, the increased number of lipid droplets in the epidermis of Snap29-deficient animals also points towards defects in lipid transportation.

\subsection{Impacts of Snap29 deficiency on autophagy}

During the time of my thesis, a role of SNAP29 in autophagy has emerged. It was recently revealed, that Snap29 plays a role in late steps of autophagosome-lysosome fusion, together with Stx 17 and VAMP8/VAMP7 in both human and fruit fly (Itakura et al, 2012; Takats et al, 2013) (see Chapter 1.6.2). Therefore, we decided to perform autophagic flux assays in addition to the characterization of the epidermal phenotype of Snap29-deficient mice. Autophagy relies on membrane fusion processes, especially regarding autophagosome biogenesis and fusion with the endocytic system (Mizushima, 2007). This process takes place under basal conditions, but it can be markedly upregulated by numerous conditions such as ER stress, as seen in Snap29-deficient fibroblasts (see Chapter 4.11). Furthermore, autophagy plays a role in the cornification of keratinocytes, which involves the degradation of intracellular constituents but it is not essential for barrier formation (Rossiter et al, 2013). However, the key regulatory mechanisms of autophagic membrane fusion as well as the involvement of autophagy in keratinocyte differentiation have not been thoroughly evaluated in the past.

Autophagic degradation is thought to be required for cellular remodeling and organelle clearance during keratinocyte differentiation (Aymard et al, 2011). Since SNAP29 is directly involved in membrane fusion of autophagosomes with lysosomes, we performed autophagic flux assays (see Chapter 1.6.1). In Snap29-deficient fibroblasts the levels of LC3B-II, a marker for autophagosomes, were increased, particularly under amino acid starvation conditions and bafilomycin A1 treatment, compared to control cells (see Chapter 4.12). Accumulation of autophagosomes, and therefore increased LC3B-II levels, may indicate an induction of autophagy or blockage of downstream steps, e.g. inhibition of autophagosomelysosome fusion (Mizushima et al, 2010) (see Chapter 1.6.1). Thus, p62 levels were examined since it correlates inversely with autophagic flux. p62 binds directly to LC 3 and ubiquitinated 
substrates and is degraded by autophagy (Bjorkoy et al, 2005). Interestingly, no accumulation of p62 was observed in Snap29-deficient fibroblasts. Our data implicate an induction of autophagy through Snap29 ablation, because no strikingly enhanced levels of p62 could be detected and a further increase of LC3B-II upon bafilomycin A1 treatment in Snap29 knockout cells.

Our data are in contrast with experiments in SNAP29 knockdown cells, in which levels of LC3-II were increased but autophagic flux was inhibited (Itakura et al, 2012). However, a study in Caenorhabditis elegans showed a significant induction of autophagy in the intestine of snap-29(RNAi) animals (Sato et al, 2011).

In addition, Drosophila melanogaster mutants for Snap29 displayed an increased number of autophagosomes and epithelial architecture defects (Morelli et al, 2014). However, Drosophila mutants in genes exclusively acting in the autophagic machinery but not in endocytic trafficking do not reveal alterations in epithelial tissue architecture or secretion defects (Chang et al, 2009; Morelli et al, 2014). Therefore, this study supports the idea that alterations in the autophagic machinery are not responsible for the epidermal phenotype of CEDNIK syndrome patients. It is rather emphasized, that the epithelial defects in Drosophila are caused by alterations in secretory trafficking. It was also suggested, that an altered autophagic pathway may be causative for the neuronal phenotype of CEDNIK syndrome patients (Morelli et al, 2014), considering that defects in autophagy contribute to neurodegeneration (reviewed by (Son et al, 2012)). In conclusion, the exact role of SNAP29 in the autophagy machinery and its contribution to a normal epidermal homeostasis warrants further investigations.

Until today, only few studies report the involvement of the autophagic pathway in epidermal keratinization and particularly gene-targeting experiments of autophagy regulators in murine models have further examined the involvement of the autophagic pathway in mammalian development (Barth et al, 2010). For example, mice in which essential genes for the autophagic machinery, such as Atg5 and Atg7, are deleted are born without an overt skin phenotype (Komatsu et al, 2005; Kuma et al, 2004). This evidence also supports the idea that perturbations in autophagy do not affect skin morphogenesis in neonatal mice. Another recent line of research proposed that autophagy is constitutively active in the epidermis but is dispensable for the formation of a functional epidermal barrier (Rossiter et al, 2013). Notably, skin graft experiments with Atg7-deficient mice predicted that autophagy has a significant role in terminal epidermal differentiation and hair growth (Yoshihara et al, 2015); however, 
epidermis specific knockout mice (K14-Cre-Atg7 f/f) did not resemble these features (Rossiter et al, 2013). These recent reports might partially be explained by the discovery of another alternative autophagy pathway, which is independent of Atg5 and Atg7, essential genes in mammalian autophagy (Nishida et al, 2009; Shimizu et al, 2010). In this cellular model, MEFs deficient for Atg5 or Atg7 were still able to form autophagosomes and autolysosomes even without LC3 lipidation. Further investigation exploring the relationship between autophagy and the subsequent differentiation of keratinocytes will be required to evaluate novel insights into these processes.

Taken together, our findings suggest that the autophagic machinery is likely affected by ablation of SNAP29; however, the neonatal murine skin phenotype especially the disturbed skin barrier function in Snap29-deficient mice is most likely not caused by alterations in the autophagic pathway. The role of SNAP29 was briefly addressed in this thesis and its emerging importance in this dynamic process requests further investigation.

\section{General remarks regarding autophagic flux assays}

Given that autophagy is a highly dynamic process that is regulated at multiple steps, it was pointed out that researchers need to carefully choose methods to monitor autophagy and that there are common pitfalls in interpreting such results. As discussed in Chapter 1.6.1, the most common approach in order to monitor autophagic activity is the measurement of LC3-II and/or p62 protein levels. However, it is important to use several approaches (pharmacological and genetic) before making final conclusions about the impact on autophagy in the respective system (Barth et al, 2010). In theory, autophagy gene knockdown/knockout represents a more specific approach than pharmacological targeting of autophagy (Mizushima \& Levine, 2010). Notably, bafilomycin A1 has in addition to the influence on autophagy an impact upon endocytic travelling (Bayer et al, 1998) and recently it was reported that this pharmacological agent does not affect the lysosomal $\mathrm{pH}$ as assumed previously (Mauvezin et al, 2015). Therefore, a more careful examination of the autophagic pathway is required for example under the treatment with other chemical inhibitors, in order to further investigate the impact of Snap29 depletion on autophagy. 


\subsection{Total knockout mice show a more pronounced phenotype}

Total as well as epidermal Snap29 knockout mice exhibited a nearly identical ichthyotic phenotype accompanied by neonatal lethality (see Figure 17). However, a detailed comparison of the phenotypes of the two knockout mouse lines indicated slight differences in epidermal differentiation as well as in hair morphogenesis. The cutaneous abnormalities of the mutant mice were more pronounced in the total knockout mice. Snap $29^{-/-}$mice displayed more organelle remnants in the stratum corneum, nucleated keratinocytes in the cornified layers (parakeratosis) (see Figure 25) and less hair follicles compared to Snap29 $9^{\mathrm{fl} / \mathrm{fl}} / \mathrm{K} 14-\mathrm{Cre}$ mice (see Chapter 4.4 and 4.7).

Interestingly, a recent study revealed that SNAP29 is directly involved in early fusion processes implicated in primary cilium assembly, and depletion of SNAP29 inhibited ciliogenesis (Lu et al, 2015). Primary cilia on the surface of mammalian cells are essential for epidermal morphogenesis (Chen et al, 2015). Furthermore, cilia are required for proper Notch-signaling and progenitor cell differentiation in skin morphogenesis. In early epidermal development, Notch-signaling via primary cilia leads to the commitment of basal keratinocytes to terminal differentiation (Ezratty et al, 2011) and cilia have been implicated in hair follicle morphogenesis, reliant upon sonic hedgehog signaling (Chen et al, 2015; Ezratty et al, 2011). Notably, ciliary-mutants displayed defects in Notch-signaling and in the commitment of progenitor cells to differentiate (Chen et al, 2015; Ezratty et al, 2011), suggesting that ciliary loss might directly affect epidermal differentiation. We assume that disturbances in primary cilia formation and the associated perturbations in progenitor cell differentiation might be responsible for the observed alleviated phenotype in total Snap29 knockout mice.

Moreover, the key prerequisite for hair follicle development relies on signaling between keratinocytes and the underlying mesenchymal fibroblasts (Mikkola, 2007; Schneider et al, 2009). A recent study revealed a role for dermal primary cilia as an essential signaling component required for regulating hair follicle morphogenesis (Lehman et al, 2009). Consistent with these data, it might be reasonably assumed that the reduction of hair follicles in total Snap29-deficient mice compared to the epidermal knockout relies on a disruption of the tightly regulated ectodermal-mesodermal interactions via early cilium assembly. At this time, little is known about the role of epidermal cilia and the involvement of SNAP29 in primary cilia assembly. Thus, the crosstalk between ciliogenesis and skin morphogenesis remains to be fully explored. 


\subsection{Summary and conclusions}

Loss-of-function mutations in the SNAP29 gene are associated with the human CEDNIK syndrome and so far, no Snap29-deficient mammalian animal models have been described for this syndrome. This work reports the generation and characterization of a total (Snap29 $9^{-/-}$) as well as keratinocyte specific (Snap29 $\left.{ }^{\mathrm{fl} / \mathrm{fl}} / \mathrm{K} 14-\mathrm{Cre}\right)$ Snap29 knockout mouse line. Both Snap29-deficient mouse models resemble the human epidermal CEDNIK syndrome phenotype and can be used as beneficial models to further enlighten the impact of SNAP29 in epidermal differentiation as well as in understanding the pathophysiological mechanisms in the human CEDNIK syndrome.

Both Snap29-deficient mouse lines showed an ichthyosiform skin phenotype accompanied by neonatal lethality. Altogether, the murine phenotype comprises epidermal differentiation defects shown by a dysregulation of keratinocyte differentiation markers as well as a reduced serine protease activity in the stratum granulosum accompanied by delayed desquamation. Furthermore, markedly decreased deposition of LB contents in mutant mice epidermis and the observation of malformed LBs indicate severe impairments in LB function due to the Snap29 knockout. Thus, my thesis presents novel evidence that strengthens a role of SNAP29 on LB maturation and secretion as demonstrated by abnormal lipid profiles, and an impaired barrier formation, which are most likely due to an impaired vesicular trafficking.

Besides the impact on terminal keratinocyte differentiation, my thesis emphasizes a role of SNAP29 in autophagy and ER stress. However, it is most likely that the observed alterations in the autophagic pathway are not primarily causative for the neonatal murine skin phenotype, especially the severe barrier perturbations in Snap29-deficient mice. Further studies will elucidate the exact role of SNAP29 in autophagy and ER stress, especially regarding normal epidermal homeostasis.

Overall, my thesis contributes to the substantial role of SNAP29 in epidermal differentiation. Further research will reveal to what extent the observed alterations in LB function in Snap29deficient epidermis are caused by direct effects on vesicular trafficking or by indirect effects due to disturbances in Golgi, ER or autophagy. A follow-up research application for further unraveling the impact of SNAP29 on autophagy and ciliogenesis is planned for submission at the Deutsche Forschungsgemeinschaft (DFG). Therefore, the isolated cells (keratinocytes and fibroblasts) from Snap $29^{-/-}$and Snap $29^{f l f l} / \mathrm{K} 14-\mathrm{Cre}$ mice can be used as a key perquisite for further investigations. 


\section{Bibliography}

Aberg KM, Man MQ, Gallo RL, Ganz T, Crumrine D, Brown BE, Choi EH, Kim DK, Schroder JM, Feingold KR, Elias PM (2008) Co-regulation and interdependence of the mammalian epidermal permeability and antimicrobial barriers. The Journal of investigative dermatology 128: 917-925

Akiyama M (2011a) The roles of ABCA12 in keratinocyte differentiation and lipid barrier formation in the epidermis. Dermato-endocrinology 3: 107-112

Akiyama M (2011b) Updated molecular genetics and pathogenesis of ichthiyoses. Nagoya J Med Sci 73: $79-90$

Anand S, Chakrabarti E, Kawamura H, Taylor CR, Maytin EV (2005) Ultraviolet light (UVB and UVA) induces the damage-responsive transcription factor $\mathrm{CHOP} /$ gadd153 in murine and human epidermis: evidence for a mechanism specific to intact skin. The Journal of investigative dermatology 125: $323-333$

Apostolovic B, Danial M, Klok HA (2010) Coiled coils: attractive protein folding motifs for the fabrication of self-assembled, responsive and bioactive materials. Chemical Society reviews 39: 35413575

Aymard E, Barruche V, Naves T, Bordes S, Closs B, Verdier M, Ratinaud MH (2011) Autophagy in human keratinocytes: an early step of the differentiation? Experimental dermatology 20: 263-268

Barlowe C, Orci L, Yeung T, Hosobuchi M, Hamamoto S, Salama N, Rexach MF, Ravazzola M, Amherdt M, Schekman R (1994) COPII: a membrane coat formed by Sec proteins that drive vesicle budding from the endoplasmic reticulum. Cell 77: 895-907

Barth S, Glick D, Macleod KF (2010) Autophagy: assays and artifacts. The Journal of pathology 221: $117-124$

Bayer N, Schober D, Prchla E, Murphy RF, Blaas D, Fuchs R (1998) Effect of bafilomycin A1 and nocodazole on endocytic transport in HeLa cells: implications for viral uncoating and infection. Journal of virology 72: 9645-9655

Behrens DT, Villone D, Koch M, Brunner G, Sorokin L, Robenek H, Bruckner-Tuderman L, Bruckner $P$, Hansen U (2012) The epidermal basement membrane is a composite of separate laminin- or collagen IV-containing networks connected by aggregated perlecan, but not by nidogens. The Journal of biological chemistry 287: 18700-18709

Belleudi F, Purpura V, Caputo S, Torrisi MR (2014) FGF7/KGF regulates autophagy in keratinocytes: A novel dual role in the induction of both assembly and turnover of autophagosomes. Autophagy 10: 803-821

Ben-Salem S, Nara S, Al-Shamsi AM, Valle D, Ali BR, Al-Gazali L (2015) New Arab family with cerebral dysgenesis, neuropathy, ichthyosis and keratoderma syndrome suggests a possible founder effect for the c.223delG mutation. The Journal of dermatology

Bjorkoy G, Lamark T, Brech A, Outzen H, Perander M, Overvatn A, Stenmark H, Johansen T (2005) p62/SQSTM1 forms protein aggregates degraded by autophagy and has a protective effect on huntingtin-induced cell death. The Journal of cell biology 171: 603-614

Blanpain C, Fuchs E (2009) Epidermal homeostasis: a balancing act of stem cells in the skin. Nature reviews Molecular cell biology 10: 207-217

Bock JB, Matern HT, Peden AA, Scheller RH (2001) A genomic perspective on membrane compartment organization. Nature 409: 839-841 
Bonifacino JS, Glick BS (2004) The mechanisms of vesicle budding and fusion. Cell 116: 153-166

Borradori L, Sonnenberg A (1999) Structure and function of hemidesmosomes: more than simple adhesion complexes. The Journal of investigative dermatology 112: 411-418

Bradford MM (1976) A rapid and sensitive method for the quantitation of microgram quantities of protein utilizing the principle of protein-dye binding. Analytical biochemistry 72: 248-254

Braff MH, Di Nardo A, Gallo RL (2005) Keratinocytes store the antimicrobial peptide cathelicidin in lamellar bodies. The Journal of investigative dermatology 124: 394-400

Brown SJ, McLean WH (2012) One remarkable molecule: filaggrin. The Journal of investigative dermatology 132: 751-762

Cai H, Reinisch K, Ferro-Novick S (2007) Coats, tethers, Rabs, and SNAREs work together to mediate the intracellular destination of a transport vesicle. Dev Cell 12: 671-682

Candi E, Schmidt R, Melino G (2005) The cornified envelope: a model of cell death in the skin. Nature reviews Molecular cell biology 6: 328-340

Cardiff RD, Miller CH, Munn RJ (2014) Manual hematoxylin and eosin staining of mouse tissue sections. Cold Spring Harbor protocols 2014: 655-658

Caubet C, Jonca N, Brattsand M, Guerrin M, Bernard D, Schmidt R, Egelrud T, Simon M, Serre G (2004) Degradation of corneodesmosome proteins by two serine proteases of the kallikrein family, SCTE/KLK5/hK5 and SCCE/KLK7/hK7. The Journal of investigative dermatology 122: 1235-1244

Celli A, Mackenzie DS, Crumrine DS, Tu CL, Hupe M, Bikle DD, Elias PM, Mauro TM (2011) Endoplasmic reticulum $\mathrm{Ca} 2+$ depletion activates XBP1 and controls terminal differentiation in keratinocytes and epidermis. The British journal of dermatology 164: 16-25

Chang YY, Juhasz G, Goraksha-Hicks P, Arsham AM, Mallin DR, Muller LK, Neufeld TP (2009) Nutrient-dependent regulation of autophagy through the target of rapamycin pathway. Biochemical Society transactions 37: 232-236

Chen J, Laclef C, Moncayo A, Snedecor ER, Yang N, Li L, Takemaru K, Paus R, SchneiderMaunoury S, Clark RA (2015) The ciliopathy gene Rpgrip11 is essential for hair follicle development. The Journal of investigative dermatology 135: 701-709

Chen YA, Scheller RH (2001) SNARE-mediated membrane fusion. Nature reviews Molecular cell biology 2: 98-106

Dale BA, Holbrook KA, Fleckman P, Kimball JR, Brumbaugh S, Sybert VP (1990) Heterogeneity in harlequin ichthyosis, an inborn error of epidermal keratinization: variable morphology and structural protein expression and a defect in lamellar granules. The Journal of investigative dermatology 94: 618

de Juanes S, Epp N, Latzko S, Neumann M, Furstenberger G, Hausser I, Stark HJ, Krieg P (2009) Development of an ichthyosiform phenotype in Alox12b-deficient mouse skin transplants. The Journal of investigative dermatology 129: 1429-1436

Deraison C, Bonnart C, Lopez F, Besson C, Robinson R, Jayakumar A, Wagberg F, Brattsand M, Hachem JP, Leonardsson G, Hovnanian A (2007) LEKTI fragments specifically inhibit KLK5, KLK7, and KLK14 and control desquamation through a pH-dependent interaction. Molecular biology of the cell 18: 3607-3619

Descargues P, Deraison C, Bonnart C, Kreft M, Kishibe M, Ishida-Yamamoto A, Elias P, Barrandon Y, Zambruno G, Sonnenberg A, Hovnanian A (2005) Spink5-deficient mice mimic Netherton 
syndrome through degradation of desmoglein 1 by epidermal protease hyperactivity. Nature genetics 37: $56-65$

Diaz G, Melis M, Batetta B, Angius F, Falchi AM (2008) Hydrophobic characterization of intracellular lipids in situ by Nile Red red/yellow emission ratio. Micron 39: 819-824

Dragatsis I, Zeitlin S (2001) A method for the generation of conditional gene repair mutations in mice. Nucleic acids research 29: E10

Eckert RL, Sturniolo MT, Broome AM, Ruse M, Rorke EA (2005) Transglutaminase function in epidermis. The Journal of investigative dermatology 124: 481-492

Eckhart L, Lippens S, Tschachler E, Declercq W (2013) Cell death by cornification. Biochim Biophys Acta 1833: 3471-3480

Eckl KM, Tidhar R, Thiele H, Oji V, Hausser I, Brodesser S, Preil ML, Onal-Akan A, Stock F, Muller D, Becker K, Casper R, Nurnberg G, Altmuller J, Nurnberg P, Traupe H, Futerman AH, Hennies HC (2013) Impaired epidermal ceramide synthesis causes autosomal recessive congenital ichthyosis and reveals the importance of ceramide acyl chain length. The Journal of investigative dermatology 133: 2202-2211

Elias PM (2012) Structure and function of the stratum corneum extracellular matrix. The Journal of investigative dermatology 132: 2131-2133

Elias PM, Ahn SK, Denda M, Brown BE, Crumrine D, Kimutai LK, Komuves L, Lee SH, Feingold KR (2002) Modulations in epidermal calcium regulate the expression of differentiation-specific markers. The Journal of investigative dermatology 119: 1128-1136

Elias PM, Choi EH (2005) Interactions among stratum corneum defensive functions. Experimental dermatology 14: 719-726

Elias PM, Cullander C, Mauro T, Rassner U, Komuves L, Brown BE, Menon GK (1998) The secretory granular cell: the outermost granular cell as a specialized secretory cell. The journal of investigative dermatology Symposium proceedings / the Society for Investigative Dermatology, Inc [and] European Society for Dermatological Research 3: 87-100

Elias PM, Fartasch M, Crumrine D, Behne M, Uchida Y, Holleran WM (2000) Origin of the corneocyte lipid envelope (CLE): observations in harlequin ichthyosis and cultured human keratinocytes. The Journal of investigative dermatology 115: 765-769

Elias PM, Williams ML, Feingold KR (2012) Abnormal barrier function in the pathogenesis of ichthyosis: therapeutic implications for lipid metabolic disorders. Clinics in dermatology 30: 311-322

Elias PM, Williams ML, Holleran WM, Jiang YJ, Schmuth M (2008) Pathogenesis of permeability barrier abnormalities in the ichthyoses: inherited disorders of lipid metabolism. Journal of lipid research 49: 697-714

Epp N, Furstenberger G, Muller K, de Juanes S, Leitges M, Hausser I, Thieme F, Liebisch G, Schmitz G, Krieg P (2007) 12R-lipoxygenase deficiency disrupts epidermal barrier function. The Journal of cell biology 177: 173-182

Ezratty EJ, Stokes N, Chai S, Shah AS, Williams SE, Fuchs E (2011) A role for the primary cilium in Notch signaling and epidermal differentiation during skin development. Cell 145: 1129-1141

Fasshauer D, Antonin W, Margittai M, Pabst S, Jahn R (1999) Mixed and non-cognate SNARE complexes. Characterization of assembly and biophysical properties. The Journal of biological chemistry 274: 15440-15446 
Fasshauer D, Sutton RB, Brunger AT, Jahn R (1998) Conserved structural features of the synaptic fusion complex: SNARE proteins reclassified as Q- and R-SNAREs. Proceedings of the National Academy of Sciences of the United States of America 95: 15781-15786

Feingold KR (2007) Thematic review series: skin lipids. The role of epidermal lipids in cutaneous permeability barrier homeostasis. Journal of lipid research 48: 2531-2546

Feingold KR (2009) The outer frontier: the importance of lipid metabolism in the skin. Journal of lipid research 50 Suppl: S417-422

Feng Y, He D, Yao Z, Klionsky DJ (2014) The machinery of macroautophagy. Cell research 24: 2441

Fuchs-Telem D, Stewart H, Rapaport D, Nousbeck J, Gat A, Gini M, Lugassy Y, Emmert S, Eckl K, Hennies HC, Sarig O, Goldsher D, Meilik B, Ishida-Yamamoto A, Horowitz M, Sprecher E (2011) CEDNIK syndrome results from loss-of-function mutations in SNAP29. The British journal of dermatology 164: 610-616

Fuchs E (1995) Keratins and the skin. Annual review of cell and developmental biology 11: 123-153

Fuchs E, Green H (1980) Changes in keratin gene expression during terminal differentiation of the keratinocyte. Cell 19: 1033-1042

Fuchs E, Raghavan S (2002) Getting under the skin of epidermal morphogenesis. Nature reviews Genetics 3: 199-209

Gelfant S (1976) The cell cycle in psoriasis: a reappraisal. The British journal of dermatology 95: 577590

Gissen P, Tee L, Johnson CA, Genin E, Caliebe A, Chitayat D, Clericuzio C, Denecke J, Di Rocco M, Fischler B, FitzPatrick D, Garcia-Cazorla A, Guyot D, Jacquemont S, Koletzko S, Leheup B, Mandel H, Sanseverino MT, Houwen RH, McKiernan PJ, Kelly DA, Maher ER (2006) Clinical and molecular genetic features of ARC syndrome. Human genetics 120: 396-409

Green KJ, Jones JC (1996) Desmosomes and hemidesmosomes: structure and function of molecular components. FASEB journal : official publication of the Federation of American Societies for Experimental Biology 10: 871-881

Guo B, Liang Q, Li L, Hu Z, Wu F, Zhang P, Ma Y, Zhao B, Kovacs AL, Zhang Z, Feng D, Chen S, Zhang H (2014) O-GlcNAc-modification of SNAP-29 regulates autophagosome maturation. Nature cell biology 16: 1215-1226

Hachem JP, Crumrine D, Fluhr J, Brown BE, Feingold KR, Elias PM (2003) pH directly regulates epidermal permeability barrier homeostasis, and stratum corneum integrity/cohesion. The Journal of investigative dermatology 121: 345-353

Hafner M, Wenk J, Nenci A, Pasparakis M, Scharffetter-Kochanek K, Smyth N, Peters T, Kess D, Holtkotter O, Shephard P, Kudlow JE, Smola H, Haase I, Schippers A, Krieg T, Muller W (2004) Keratin 14 Cre transgenic mice authenticate keratin 14 as an oocyte-expressed protein. Genesis 38: 176-181

Hailey DW, Rambold AS, Satpute-Krishnan P, Mitra K, Sougrat R, Kim PK, Lippincott-Schwartz J (2010) Mitochondria supply membranes for autophagosome biogenesis during starvation. Cell 141: 656-667

Halprin KM (1972) Epidermal "turnover time"--a re-examination. The British journal of dermatology 86: $14-19$ 
Hamasaki M, Furuta N, Matsuda A, Nezu A, Yamamoto A, Fujita N, Oomori H, Noda T, Haraguchi T, Hiraoka Y, Amano A, Yoshimori T (2013) Autophagosomes form at ER-mitochondria contact sites. Nature 495: 389-393

Hapala I, Marza E, Ferreira T (2011) Is fat so bad? Modulation of endoplasmic reticulum stress by lipid droplet formation. Biology of the cell / under the auspices of the European Cell Biology Organization 103: 271-285

Hayashi T, McMahon H, Yamasaki S, Binz T, Hata Y, Sudhof TC, Niemann H (1994) Synaptic vesicle membrane fusion complex: action of clostridial neurotoxins on assembly. The EMBO journal 13: 5051-5061

Hennings H, Michael D, Cheng C, Steinert P, Holbrook K, Yuspa SH (1980) Calcium regulation of growth and differentiation of mouse epidermal cells in culture. Cell 19: 245-254

Hershkovitz D, Mandel H, Ishida-Yamamoto A, Chefetz I, Hino B, Luder A, Indelman M, Bergman R, Sprecher E (2008) Defective lamellar granule secretion in arthrogryposis, renal dysfunction, and cholestasis syndrome caused by a mutation in VPS33B. Archives of dermatology 144: 334-340

Hohenstein AC, Roche PA (2001) SNAP-29 is a promiscuous syntaxin-binding SNARE. Biochemical and biophysical research communications 285: 167-171

Holt M, Varoqueaux F, Wiederhold K, Takamori S, Urlaub H, Fasshauer D, Jahn R (2006) Identification of SNAP-47, a novel Qbc-SNARE with ubiquitous expression. The Journal of biological chemistry 281: 17076-17083

Irvine AD, McLean WH, Leung DY (2011) Filaggrin mutations associated with skin and allergic diseases. The New England journal of medicine 365: 1315-1327

Ishida-Yamamoto A, Hohl D, Roop DR, lizuka H, Eady RA (1993) Loricrin immunoreactivity in human skin: localization to specific granules (L-granules) in acrosyringia. Archives of dermatological research 285: 491-498

Ishida-Yamamoto A, Kishibe M (2011) Involvement of corneodesmosome degradation and lamellar granule transportation in the desquamation process. Medical molecular morphology 44: 1-6

Ishida-Yamamoto A, Simon M, Kishibe M, Miyauchi Y, Takahashi H, Yoshida S, O'Brien TJ, Serre G, lizuka H (2004) Epidermal lamellar granules transport different cargoes as distinct aggregates. The Journal of investigative dermatology 122: 1137-1144

Itakura E, Kishi-Itakura C, Mizushima N (2012) The hairpin-type tail-anchored SNARE syntaxin 17 targets to autophagosomes for fusion with endosomes/lysosomes. Cell 151: 1256-1269

Itakura E, Mizushima N (2010) Characterization of autophagosome formation site by a hierarchical analysis of mammalian Atg proteins. Autophagy 6: 764-776

Jahn R, Scheller RH (2006) SNAREs--engines for membrane fusion. Nature reviews Molecular cell biology 7: 631-643

Jennemann R, Sandhoff R, Langbein L, Kaden S, Rothermel U, Gallala H, Sandhoff K, Wiegandt H, Grone HJ (2007) Integrity and barrier function of the epidermis critically depend on glucosylceramide synthesis. The Journal of biological chemistry 282: 3083-3094

Jonca N, Leclerc EA, Caubet C, Simon M, Guerrin M, Serre G (2011) Corneodesmosomes and corneodesmosin: from the stratum corneum cohesion to the pathophysiology of genodermatoses. European journal of dermatology : EJD 21 Suppl 2: 35-42 
Kadowaki H, Nishitoh H (2013) Signaling pathways from the endoplasmic reticulum and their roles in disease. Genes 4: 306-333

Kalinin AE, Kajava AV, Steinert PM (2002) Epithelial barrier function: assembly and structural features of the cornified cell envelope. BioEssays : news and reviews in molecular, cellular and developmental biology 24: 789-800

Kang J, Bai Z, Zegarek MH, Grant BD, Lee J (2011) Essential roles of snap-29 in C. elegans. Developmental biology 355: 77-88

Karlsson U, Schultz RL (1965) Fixation of the Central Nervous System from Electron Microscopy by Aldehyde Perfusion. I. Preservation with Aldehyde Perfusates Versus Direct Perfusion with Osmium Tetroxide with Special Reference to Membranes and the Extracellular Space. Journal of ultrastructure research 12: 160-186

Klionsky DJ, Elazar Z, Seglen PO, Rubinsztein DC (2008) Does bafilomycin A1 block the fusion of autophagosomes with lysosomes? Autophagy 4: 849-850

Komatsu M, Waguri S, Ueno T, Iwata J, Murata S, Tanida I, Ezaki J, Mizushima N, Ohsumi Y, Uchiyama Y, Kominami E, Tanaka K, Chiba T (2005) Impairment of starvation-induced and constitutive autophagy in Atg7-deficient mice. The Journal of cell biology 169: 425-434

Kominami E, Hashida S, Khairallah EA, Katunuma N (1983) Sequestration of cytoplasmic enzymes in an autophagic vacuole-lysosomal system induced by injection of leupeptin. The Journal of biological chemistry 258: 6093-6100

Kopitz J, Kisen GO, Gordon PB, Bohley P, Seglen PO (1990) Nonselective autophagy of cytosolic enzymes by isolated rat hepatocytes. The Journal of cell biology 111: 941-953

Kuma A, Hatano M, Matsui M, Yamamoto A, Nakaya H, Yoshimori T, Ohsumi Y, Tokuhisa T, Mizushima N (2004) The role of autophagy during the early neonatal starvation period. Nature 432: $1032-1036$

Lakso M, Pichel JG, Gorman JR, Sauer B, Okamoto Y, Lee E, Alt FW, Westphal H (1996) Efficient in vivo manipulation of mouse genomic sequences at the zygote stage. Proceedings of the National Academy of Sciences of the United States of America 93: 5860-5865

Lamb CA, Yoshimori T, Tooze SA (2013) The autophagosome: origins unknown, biogenesis complex. Nature reviews Molecular cell biology 14: 759-774

Lane EB, Bartek J, Purkis PE, Leigh IM (1985) Keratin antigens in differentiating skin. Annals of the New York Academy of Sciences 455: 241-258

Lee SH, Elias PM, Proksch E, Menon GK, Mao-Quiang M, Feingold KR (1992) Calcium and potassium are important regulators of barrier homeostasis in murine epidermis. The Journal of clinical investigation 89: 530-538

Lehman JM, Laag E, Michaud EJ, Yoder BK (2009) An essential role for dermal primary cilia in hair follicle morphogenesis. The Journal of investigative dermatology 129: 438-448

Leigh IM, Purkis PE, Whitehead P, Lane EB (1993) Monospecific monoclonal antibodies to keratin 1 carboxy terminal (synthetic peptide) and to keratin 10 as markers of epidermal differentiation. The British journal of dermatology 129: 110-119 
Letourneur F, Gaynor EC, Hennecke S, Demolliere C, Duden R, Emr SD, Riezman H, Cosson P (1994) Coatomer is essential for retrieval of dilysine-tagged proteins to the endoplasmic reticulum. Cell 79: 1199-1207

Leyvraz C, Charles RP, Rubera I, Guitard M, Rotman S, Breiden B, Sandhoff K, Hummler E (2005) The epidermal barrier function is dependent on the serine protease CAP1/Prss8. The Journal of cell biology 170: 487-496

Li F, Pincet F, Perez E, Eng WS, Melia TJ, Rothman JE, Tareste D (2007) Energetics and dynamics of SNAREpin folding across lipid bilayers. Nature structural \& molecular biology 14: 890-896

Li Q, Frank M, Akiyama M, Shimizu H, Ho SY, Thisse C, Thisse B, Sprecher E, Uitto J (2011) Abca12-mediated lipid transport and Snap29-dependent trafficking of lamellar granules are crucial for epidermal morphogenesis in a zebrafish model of ichthyosis. Disease models \& mechanisms 4: 777785

Lin RC, Scheller RH (1997) Structural organization of the synaptic exocytosis core complex. Neuron 19: 1087-1094

Lippens S, Kockx M, Knaapen M, Mortier L, Polakowska R, Verheyen A, Garmyn M, Zwijsen A, Formstecher P, Huylebroeck D, Vandenabeele P, Declercq W (2000) Epidermal differentiation does not involve the pro-apoptotic executioner caspases, but is associated with caspase-14 induction and processing. Cell death and differentiation 7: 1218-1224

List K, Haudenschild CC, Szabo R, Chen W, Wahl SM, Swaim W, Engelholm LH, Behrendt N, Bugge TH (2002) Matriptase/MT-SP1 is required for postnatal survival, epidermal barrier function, hair follicle development, and thymic homeostasis. Oncogene 21: 3765-3779

Littleton JT, Barnard RJ, Titus SA, Slind J, Chapman ER, Ganetzky B (2001) SNARE-complex disassembly by NSF follows synaptic-vesicle fusion. Proceedings of the National Academy of Sciences of the United States of America 98: 12233-12238

Lloyd C, Yu QC, Cheng J, Turksen K, Degenstein L, Hutton E, Fuchs E (1995) The basal keratin network of stratified squamous epithelia: defining K15 function in the absence of K14. The Journal of cell biology 129: 1329-1344

Lu Q, Insinna C, Ott C, Stauffer J, Pintado PA, Rahajeng J, Baxa U, Walia V, Cuenca A, Hwang YS, Daar IO, Lopes S, Lippincott-Schwartz J, Jackson PK, Caplan S, Westlake CJ (2015) Early steps in primary cilium assembly require EHD1/EHD3-dependent ciliary vesicle formation. Nature cell biology 17: 228-240

Lundstrom A, Serre G, Haftek M, Egelrud T (1994) Evidence for a role of corneodesmosin, a protein which may serve to modify desmosomes during cornification, in stratum corneum cell cohesion and desquamation. Archives of dermatological research 286: 369-375

Madison KC (2003) Barrier function of the skin: "la raison d'etre" of the epidermis. The Journal of investigative dermatology 121: 231-241

Madison KC, Sando GN, Howard EJ, True CA, Gilbert D, Swartzendruber DC, Wertz PW (1998) Lamellar granule biogenesis: a role for ceramide glucosyltransferase, lysosomal enzyme transport, and the Golgi. The journal of investigative dermatology Symposium proceedings / the Society for Investigative Dermatology, Inc [and] European Society for Dermatological Research 3: 80-86

Matsui T, Amagai M (2015) Dissecting the formation, structure and barrier function of the stratum corneum. International immunology 27: 269-280 
Mauro T, Bench G, Sidderas-Haddad E, Feingold K, Elias P, Cullander C (1998) Acute barrier perturbation abolishes the $\mathrm{Ca} 2+$ and $\mathrm{K}+$ gradients in murine epidermis: quantitative measurement using PIXE. The Journal of investigative dermatology 111: 1198-1201

Mauvezin C, Nagy P, Juhasz G, Neufeld TP (2015) Autophagosome-lysosome fusion is independent of V-ATPase-mediated acidification. Nature communications 6: 7007

McDonald-McGinn DM, Fahiminiya S, Revil T, Nowakowska BA, Suhl J, Bailey A, Mlynarski E, Lynch DR, Yan AC, Bilaniuk LT, Sullivan KE, Warren ST, Emanuel BS, Vermeesch JR, Zackai EH, Jerome-Majewska LA (2013) Hemizygous mutations in SNAP29 unmask autosomal recessive conditions and contribute to atypical findings in patients with 22q11.2DS. J Med Genet 50: 80-90

McGowan K, Coulombe PA (1998) The wound repair-associated keratins 6, 16, and 17. Insights into the role of intermediate filaments in specifying keratinocyte cytoarchitecture. Sub-cellular biochemistry 31: 173-204

Mehrel T, Hohl D, Rothnagel JA, Longley MA, Bundman D, Cheng C, Lichti U, Bisher ME, Steven AC, Steinert PM, et al. (1990) Identification of a major keratinocyte cell envelope protein, loricrin. Cell 61: 1103-1112

Menon GK, Elias PM (1991) Ultrastructural localization of calcium in psoriatic and normal human epidermis. Archives of dermatology 127: 57-63

Menon GK, Feingold KR, Elias PM (1992) Lamellar body secretory response to barrier disruption. The Journal of investigative dermatology 98: 279-289

Menon GK, Price LF, Bommannan B, Elias PM, Feingold KR (1994) Selective obliteration of the epidermal calcium gradient leads to enhanced lamellar body secretion. The Journal of investigative dermatology 102: 789-795

Merritt AJ, Berika MY, Zhai W, Kirk SE, Ji B, Hardman MJ, Garrod DR (2002) Suprabasal desmoglein 3 expression in the epidermis of transgenic mice results in hyperproliferation and abnormal differentiation. Molecular and cellular biology 22: 5846-5858

Mikkola ML (2007) Genetic basis of skin appendage development. Seminars in cell \& developmental biology 18: 225-236

Mildner M, Jin J, Eckhart L, Kezic S, Gruber F, Barresi C, Stremnitzer C, Buchberger M, Mlitz V, Ballaun C, Sterniczky B, Fodinger D, Tschachler E (2010) Knockdown of filaggrin impairs diffusion barrier function and increases UV sensitivity in a human skin model. The Journal of investigative dermatology 130: 2286-2294

Milner ME, O'Guin WM, Holbrook KA, Dale BA (1992) Abnormal lamellar granules in harlequin ichthyosis. The Journal of investigative dermatology 99: 824-829

Misura KM, Gonzalez LC, Jr., May AP, Scheller RH, Weis WI (2001a) Crystal structure and biophysical properties of a complex between the N-terminal SNARE region of SNAP25 and syntaxin 1a. The Journal of biological chemistry 276: 41301-41309

Misura KM, Scheller RH, Weis WI (2001b) Self-association of the H3 region of syntaxin 1A. Implications for intermediates in SNARE complex assembly. The Journal of biological chemistry 276: 13273-13282

Mizushima N (2005) The pleiotropic role of autophagy: from protein metabolism to bactericide. Cell death and differentiation 12 Suppl 2: 1535-1541 
Mizushima N (2007) Autophagy: process and function. Genes \& development 21: 2861-2873

Mizushima N (2014) Sugar modification inhibits autophagosome-lysosome fusion. Nature cell biology 16: $1132-1133$

Mizushima N, Levine B (2010) Autophagy in mammalian development and differentiation. Nature cell biology 12: 823-830

Mizushima N, Yoshimori T, Levine B (2010) Methods in mammalian autophagy research. Cell 140: 313-326

Mobius W, Cooper B, Kaufmann WA, Imig C, Ruhwedel T, Snaidero N, Saab AS, Varoqueaux F (2010) Electron microscopy of the mouse central nervous system. Methods in cell biology 96: 475-512

Moll R, Divo M, Langbein L (2008) The human keratins: biology and pathology. Histochemistry and cell biology 129: 705-733

Moll R, Franke WW, Schiller DL, Geiger B, Krepler R (1982) The catalog of human cytokeratins: patterns of expression in normal epithelia, tumors and cultured cells. Cell 31: 11-24

Montpetit A, Cote S, Brustein E, Drouin CA, Lapointe L, Boudreau M, Meloche C, Drouin R, Hudson TJ, Drapeau P, Cossette P (2008) Disruption of AP1S1, causing a novel neurocutaneous syndrome, perturbs development of the skin and spinal cord. PLoS genetics 4: e1000296

Morelli E, Ginefra P, Mastrodonato V, Beznoussenko GV, Rusten TE, Bilder D, Stenmark H, Mironov AA, Vaccari T (2014) Multiple functions of the SNARE protein Snap29 in autophagy, endocytic, and exocytic trafficking during epithelial formation in Drosophila. Autophagy 10: 22512268

Moriyama M, Moriyama H, Uda J, Matsuyama A, Osawa M, Hayakawa T (2014) BNIP3 plays crucial roles in the differentiation and maintenance of epidermal keratinocytes. The Journal of investigative dermatology 134: 1627-1635

Mullis K, Faloona F, Scharf S, Saiki R, Horn G, Erlich H (1986) Specific enzymatic amplification of DNA in vitro: the polymerase chain reaction. Cold Spring Harbor symposia on quantitative biology 51 Pt 1: 263-273

Niemi KM, Kanerva L, Kuokkanen K, Ignatius J (1994) Clinical, light and electron microscopic features of recessive congenital ichthyosis type I. The British journal of dermatology 130: 626-633

Nishida Y, Arakawa S, Fujitani K, Yamaguchi H, Mizuta T, Kanaseki T, Komatsu M, Otsu K, Tsujimoto Y, Shimizu S (2009) Discovery of Atg5/Atg7-independent alternative macroautophagy. Nature 461: 654-658

Nishifuji K, Yoon JS (2013) The stratum corneum: the rampart of the mammalian body. Vet Dermatol 24: 60-72 e15-66

Norlen L (2001) Skin barrier formation: the membrane folding model. The Journal of investigative dermatology 117: 823-829

Ny A, Egelrud T (2004) Epidermal hyperproliferation and decreased skin barrier function in mice overexpressing stratum corneum chymotryptic enzyme. Acta dermato-venereologica 84: 18-22

Odell ID, Cook D (2013) Immunofluorescence techniques. The Journal of investigative dermatology 133: e4 
Ogata M, Hino S, Saito A, Morikawa K, Kondo S, Kanemoto S, Murakami T, Taniguchi M, Tanii I, Yoshinaga K, Shiosaka S, Hammarback JA, Urano F, Imaizumi K (2006) Autophagy is activated for cell survival after endoplasmic reticulum stress. Molecular and cellular biology 26: 9220-9231

Ohsaki Y, Shinohara Y, Suzuki M, Fujimoto T (2010) A pitfall in using BODIPY dyes to label lipid droplets for fluorescence microscopy. Histochemistry and cell biology 133: 477-480

Oji V, Traupe H (2006) Ichthyoses: differential diagnosis and molecular genetics. European journal of dermatology : EJD 16: 349-359

Ossig R, Schmitt HD, de Groot B, Riedel D, Keranen S, Ronne H, Grubmuller H, Jahn R (2000) Exocytosis requires asymmetry in the central layer of the SNARE complex. The EMBO journal 19: 6000-6010

Pelham HR (2001) SNAREs and the specificity of membrane fusion. Trends in cell biology 11: 99-101

Poirier MA, Hao JC, Malkus PN, Chan C, Moore MF, King DS, Bennett MK (1998) Protease resistance of syntaxin.SNAP-25.VAMP complexes. Implications for assembly and structure. The Journal of biological chemistry 273: 11370-11377

Potten CS (1981) Cell replacement in epidermis (keratopoiesis) via discrete units of proliferation. International review of cytology 69: 271-318

Potten CS, Saffhill R, Maibach HI (1987) Measurement of the transit time for cells through the epidermis and stratum corneum of the mouse and guinea-pig. Cell Tissue Kinet 20: 461-472

Presland RB, Boggess D, Lewis SP, Hull C, Fleckman P, Sundberg JP (2000) Loss of normal profilaggrin and filaggrin in flaky tail (ft/ft) mice: an animal model for the filaggrin-deficient skin disease ichthyosis vulgaris. The Journal of investigative dermatology 115: 1072-1081

Proksch E, Brandner JM, Jensen JM (2008) The skin: an indispensable barrier. Experimental dermatology 17: 1063-1072

Prose PH, Friedman-Kien AE, Neistein S (1967) Ultrastructural studies of organ cultures of adult human skin. In vitro growth and keratinization of epidermal cells. Laboratory investigation; a journal of technical methods and pathology 17: 693-716

Qiu W, Li X, Tang H, Huang AS, Panteleyev AA, Owens DM, Su GH (2011) Conditional activin receptor type 1B (Acvr1b) knockout mice reveal hair loss abnormality. The Journal of investigative dermatology 131: 1067-1076

Radner FP, Streith IE, Schoiswohl G, Schweiger M, Kumari M, Eichmann TO, Rechberger G, Koefeler HC, Eder S, Schauer S, Theussl HC, Preiss-Landl K, Lass A, Zimmermann R, Hoefler G, Zechner R, Haemmerle G (2010) Growth retardation, impaired triacylglycerol catabolism, hepatic steatosis, and lethal skin barrier defect in mice lacking comparative gene identification-58 (CGI-58). The Journal of biological chemistry 285: 7300-7311

Rapaport D, Lugassy Y, Sprecher E, Horowitz M (2010) Loss of SNAP29 impairs endocytic recycling and cell motility. PloS one 5: e9759

Rassner U, Feingold KR, Crumrine DA, Elias PM (1999) Coordinate assembly of lipids and enzyme proteins into epidermal lamellar bodies. Tissue \& cell 31: 489-498

Ravikumar B, Moreau K, Jahreiss L, Puri C, Rubinsztein DC (2010) Plasma membrane contributes to the formation of pre-autophagosomal structures. Nature cell biology 12: 747-757 
Rawlings AV, Harding CR (2004) Moisturization and skin barrier function. Dermatologic therapy 17 Suppl 1: 43-48

Raymond AA, Gonzalez de Peredo A, Stella A, Ishida-Yamamoto A, Bouyssie D, Serre G, Monsarrat B, Simon M (2008) Lamellar bodies of human epidermis: proteomics characterization by high throughput mass spectrometry and possible involvement of CLIP-170 in their trafficking/secretion. Mol Cell Proteomics 7: 2151-2175

Roop DR, Hawley-Nelson P, Cheng CK, Yuspa SH (1983) Keratin gene expression in mouse epidermis and cultured epidermal cells. Proceedings of the National Academy of Sciences of the United States of America 80: 716-720

Rossiter H, Konig U, Barresi C, Buchberger M, Ghannadan M, Zhang CF, Mlitz V, Gmeiner R, Sukseree S, Fodinger D, Eckhart L, Tschachler E (2013) Epidermal keratinocytes form a functional skin barrier in the absence of Atg7 dependent autophagy. Journal of dermatological science 71: 67-75

Rotem-Yehudar R, Galperin E, Horowitz M (2001) Association of insulin-like growth factor 1 receptor with EHD1 and SNAP29. The Journal of biological chemistry 276: 33054-33060

Rubinsztein DC, Cuervo AM, Ravikumar B, Sarkar S, Korolchuk V, Kaushik S, Klionsky DJ (2009) In search of an "autophagomometer". Autophagy 5: 585-589

Sakai K, Akiyama M, Sugiyama-Nakagiri Y, McMillan JR, Sawamura D, Shimizu H (2007) Localization of ABCA12 from Golgi apparatus to lamellar granules in human upper epidermal keratinocytes. Experimental dermatology 16: 920-926

Samali A, Fitzgerald U, Deegan S, Gupta S (2010) Methods for monitoring endoplasmic reticulum stress and the unfolded protein response. International journal of cell biology 2010: 830307

Samuelov L, Sprecher E (2014) Inherited desmosomal disorders. Cell and tissue research

Sato M, Saegusa K, Sato K, Hara T, Harada A, Sato K (2011) Caenorhabditis elegans SNAP-29 is required for organellar integrity of the endomembrane system and general exocytosis in intestinal epithelial cells. Molecular biology of the cell 22: 2579-2587

Schardt A, Brinkmann BG, Mitkovski M, Sereda MW, Werner HB, Nave KA (2009) The SNARE protein SNAP-29 interacts with the GTPase Rab3A: Implications for membrane trafficking in myelinating glia. Journal of neuroscience research 87: 3465-3479

Scharfenberger L, Hennerici T, Kiraly G, Kitzmuller S, Vernooij M, Zielinski JG (2014) Transgenic mouse technology in skin biology: generation of complete or tissue-specific knockout mice. The Journal of investigative dermatology 134: e16

Schiller S, Seebode C, Wieser G, Goebbels S, Möbius W, Horowitz M, Sarig O, Sprecher E, Emmert S (2015) Establishment of two mouse models for CEDNIK syndrome reveals the pivotal role of SNAP29 in epidermal differentiation. The Journal of investigative dermatolog, in press

Schmuth M (2003) Dropping lipids for epidermal defense. The Journal of investigative dermatology 121: $x i$

Schmuth M, Martinz V, Janecke AR, Fauth C, Schossig A, Zschocke J, Gruber R (2013) Inherited ichthyoses/generalized Mendelian disorders of cornification. Eur J Hum Genet 21: 123-133

Schneider MR, Schmidt-Ullrich R, Paus R (2009) The hair follicle as a dynamic miniorgan. Current biology : $C B$ 19: R132-142 
Scholzen T, Gerdes J (2000) The Ki-67 protein: from the known and the unknown. Journal of cellular physiology 182: 311-322

Scott CA, Rajpopat S, Di WL (2013) Harlequin ichthyosis: ABCA12 mutations underlie defective lipid transport, reduced protease regulation and skin-barrier dysfunction. Cell and tissue research 351: 281-288

Segre J (2003) Complex redundancy to build a simple epidermal permeability barrier. Current opinion in cell biology 15: 776-782

Serre G, Mils V, Haftek M, Vincent C, Croute F, Reano A, Ouhayoun JP, Bettinger S, Soleilhavoup JP (1991) Identification of late differentiation antigens of human cornified epithelia, expressed in reorganized desmosomes and bound to cross-linked envelope. The Journal of investigative dermatology 97: 1061-1072

Shimizu S, Arakawa S, Nishida Y (2010) Autophagy takes an alternative pathway. Autophagy 6: 290291

Smith FJ, Irvine AD, Terron-Kwiatkowski A, Sandilands A, Campbell LE, Zhao Y, Liao H, Evans AT, Goudie DR, Lewis-Jones S, Arseculeratne G, Munro CS, Sergeant A, O'Regan G, Bale SJ, Compton JG, DiGiovanna JJ, Presland RB, Fleckman P, McLean WH (2006) Loss-of-function mutations in the gene encoding filaggrin cause ichthyosis vulgaris. Nature genetics 38: 337-342

Smyth I, Hacking DF, Hilton AA, Mukhamedova N, Meikle PJ, Ellis S, Satterley K, Collinge JE, de Graaf CA, Bahlo M, Sviridov D, Kile BT, Hilton DJ (2008) A mouse model of harlequin ichthyosis delineates a key role for Abca12 in lipid homeostasis. PLoS genetics 4: e1000192

Sollner T, Bennett MK, Whiteheart SW, Scheller RH, Rothman JE (1993) A protein assemblydisassembly pathway in vitro that may correspond to sequential steps of synaptic vesicle docking, activation, and fusion. Cell 75: 409-418

Son JH, Shim JH, Kim KH, Ha JY, Han JY (2012) Neuronal autophagy and neurodegenerative diseases. Experimental \& molecular medicine 44: 89-98

Sprecher E, Ishida-Yamamoto A, Mizrahi-Koren M, Rapaport D, Goldsher D, Indelman M, Topaz O, Chefetz I, Keren H, O'Brien T J, Bercovich D, Shalev S, Geiger D, Bergman R, Horowitz M, Mandel H (2005) A mutation in SNAP29, coding for a SNARE protein involved in intracellular trafficking, causes a novel neurocutaneous syndrome characterized by cerebral dysgenesis, neuropathy, ichthyosis, and palmoplantar keratoderma. Am J Hum Genet 77: 242-251

Sridharan G, Shankar AA (2012) Toluidine blue: A review of its chemistry and clinical utility. Journal of oral and maxillofacial pathology : JOMFP 16: 251-255

Steegmaier M, Yang B, Yoo JS, Huang B, Shen M, Yu S, Luo Y, Scheller RH (1998) Three novel proteins of the syntaxin/SNAP-25 family. The Journal of biological chemistry 273: 34171-34179

Steinert PM, Marekov LN (1995) The proteins elafin, filaggrin, keratin intermediate filaments, loricrin, and small proline-rich proteins 1 and 2 are isodipeptide cross-linked components of the human epidermal cornified cell envelope. The Journal of biological chemistry 270: 17702-17711

Steinert PM, Marekov LN (1999) Initiation of assembly of the cell envelope barrier structure of stratified squamous epithelia. Molecular biology of the cell 10: 4247-4261

Steven AC, Bisher ME, Roop DR, Steinert PM (1990) Biosynthetic pathways of filaggrin and loricrin-two major proteins expressed by terminally differentiated epidermal keratinocytes. Journal of structural biology 104: 150-162 
Stoler A, Kopan R, Duvic M, Fuchs E (1988) Use of monospecific antisera and cRNA probes to localize the major changes in keratin expression during normal and abnormal epidermal differentiation. The Journal of cell biology 107: 427-446

Stoorvogel W, Oorschot V, Geuze HJ (1996) A novel class of clathrin-coated vesicles budding from endosomes. The Journal of cell biology 132: 21-33

Su Q, Mochida S, Tian JH, Mehta R, Sheng ZH (2001) SNAP-29: a general SNARE protein that inhibits SNARE disassembly and is implicated in synaptic transmission. Proceedings of the National Academy of Sciences of the United States of America 98: 14038-14043

Sugiura K (2013) Unfolded protein response in keratinocytes: impact on normal and abnormal keratinization. Journal of dermatological science 69: 181-186

Sugiura K, Muro Y, Futamura K, Matsumoto K, Hashimoto N, Nishizawa Y, Nagasaka T, Saito H, Tomita Y, Usukura J (2009) The unfolded protein response is activated in differentiating epidermal keratinocytes. The Journal of investigative dermatology 129: 2126-2135

Sybert VP, Dale BA, Holbrook KA (1985) Ichthyosis vulgaris: identification of a defect in synthesis of filaggrin correlated with an absence of keratohyaline granules. The Journal of investigative dermatology 84: 191-194

Takats S, Nagy P, Varga A, Pircs K, Karpati M, Varga K, Kovacs AL, Hegedus K, Juhasz G (2013) Autophagosomal Syntaxin17-dependent lysosomal degradation maintains neuronal function in Drosophila. The Journal of cell biology 201: 531-539

Taljebini M, Warren R, Mao-Oiang M, Lane E, Elias PM, Feingold KR (1996) Cutaneous permeability barrier repair following various types of insults: kinetics and effects of occlusion. Skin pharmacology : the official journal of the Skin Pharmacology Society 9: 111-119

Tanida I, Minematsu-Ikeguchi N, Ueno T, Kominami E (2005) Lysosomal turnover, but not a cellular level, of endogenous LC3 is a marker for autophagy. Autophagy 1: 84-91

Thyssen JP, Godoy-Gijon E, Elias PM (2013) Ichthyosis vulgaris: the filaggrin mutation disease. The British journal of dermatology 168: 1155-1166

Traupe H, Fischer J, Oji V (2014) Nonsyndromic types of ichthyoses - an update. Journal der Deutschen Dermatologischen Gesellschaft = Journal of the German Society of Dermatology : JDDG 12: $109-121$

Tybulewicz VL, Tremblay ML, LaMarca ME, Willemsen R, Stubblefield BK, Winfield S, Zablocka B, Sidransky E, Martin BM, Huang SP, et al. (1992) Animal model of Gaucher's disease from targeted disruption of the mouse glucocerebrosidase gene. Nature 357: 407-410

Uemura T, Ueda T, Ohniwa RL, Nakano A, Takeyasu K, Sato MH (2004) Systematic analysis of SNARE molecules in Arabidopsis: dissection of the post-Golgi network in plant cells. Cell structure and function 29: 49-65

Ungar D, Hughson FM (2003) SNARE protein structure and function. Annual review of cell and developmental biology 19: 493-517

Vallet-Pichard A, Mallet V, Pol S (2012) Nonalcoholic fatty liver disease and HIV infection. Seminars in liver disease 32: 158-166

van der Vaart A, Reggiori F (2010) The Golgi complex as a source for yeast autophagosomal membranes. Autophagy 6: 800-801 
Wang X, Zinkel S, Polonsky K, Fuchs E (1997) Transgenic studies with a keratin promoter-driven growth hormone transgene: prospects for gene therapy. Proceedings of the National Academy of Sciences of the United States of America 94: 219-226

Waters MG, Serafini T, Rothman JE (1991) 'Coatomer': a cytosolic protein complex containing subunits of non-clathrin-coated Golgi transport vesicles. Nature 349: 248-251

Wertz PW (2000) Lipids and barrier function of the skin. Acta dermato-venereologica Supplementum 208: $7-11$

Wesolowski J, Caldwell V, Paumet F (2012) A novel function for SNAP29 (synaptosomal-associated protein of $29 \mathrm{kDa}$ ) in mast cell phagocytosis. PloS one 7: e49886

Whyte JR, Munro S (2002) Vesicle tethering complexes in membrane traffic. Journal of cell science 115: $2627-2637$

Wiederhold K, Fasshauer D (2009) Is assembly of the SNARE complex enough to fuel membrane fusion? The Journal of biological chemistry 284: 13143-13152

Wilfling F, Haas JT, Walther TC, Farese RV, Jr. (2014) Lipid droplet biogenesis. Current opinion in cell biology 29: 39-45

Wong SH, Xu Y, Zhang T, Griffiths G, Lowe SL, Subramaniam VN, Seow KT, Hong W (1999) GS32, a novel Golgi SNARE of $32 \mathrm{kDa}$, interacts preferentially with syntaxin 6. Molecular biology of the cell 10: 119-134

Xu H, Mohtashami M, Stewart B, Boulianne G, Trimble WS (2014) Drosophila SNAP-29 is an essential SNARE that binds multiple proteins involved in membrane traffic. PloS one 9: e91471

Yamamoto A, Tagawa Y, Yoshimori T, Moriyama Y, Masaki R, Tashiro Y (1998) Bafilomycin A1 prevents maturation of autophagic vacuoles by inhibiting fusion between autophagosomes and lysosomes in rat hepatoma cell line, H-4-II-E cells. Cell structure and function 23: 33-42

Yanagi T, Akiyama M, Nishihara H, Sakai K, Nishie W, Tanaka S, Shimizu H (2008) Harlequin ichthyosis model mouse reveals alveolar collapse and severe fetal skin barrier defects. Human molecular genetics 17: 3075-3083

Yang B, Gonzalez L, Jr., Prekeris R, Steegmaier M, Advani RJ, Scheller RH (1999) SNARE interactions are not selective. Implications for membrane fusion specificity. The Journal of biological chemistry 274: 5649-5653

Yoneda K, Hohl D, McBride OW, Wang M, Cehrs KU, Idler WW, Steinert PM (1992) The human loricrin gene. The Journal of biological chemistry 267: 18060-18066

Yoshihara N, Ueno T, Takagi A, Oliva Trejo JA, Haruna K, Suga Y, Komatsu M, Tanaka K, Ikeda S (2015) The significant role of autophagy in the granular layer in normal skin differentiation and hair growth. Archives of dermatological research 307: 159-169

Yuspa SH, Kilkenny AE, Steinert PM, Roop DR (1989) Expression of murine epidermal differentiation markers is tightly regulated by restricted extracellular calcium concentrations in vitro. The Journal of cell biology 109: 1207-1217

Zhang J, Naslavsky N, Caplan S (2012) EHDs meet the retromer: Complex regulation of retrograde transport. Cellular logistics 2: 161-165

Zhou Y, Zhang J (2014) Arthrogryposis-renal dysfunction-cholestasis (ARC) syndrome: from molecular genetics to clinical features. Italian journal of pediatrics 40: 77 


\section{Curriculum vitae}

\section{Personal details}

$\begin{array}{ll}\text { Name } & \text { Christina Seebode } \\ & \text { born } 17^{\text {th }} \text { April } 1986 \text { in Göttingen, Germany } \\ & \text { Department of Dermatology, Venereology and Allergology } \\ & \text { University Medical Center Göttingen } \\ & \text { Robert-Koch Straße } 40 \\ & 37075 \text { Göttingen }\end{array}$

\section{Education}

August 2015

Research associate at the University Medical Center Rostock, Germany, (responsible for the laboratory relocation from Göttingen to Rostock)

Department of Dermatology and Venereology

2012-2015

2011-2012

$\mathrm{PhD}$ Thesis at the Department of Dermatology, Venereology and Allergology, supervised by Prof. Dr. med. Steffen Emmert and Dr.

Stina Schiller with the title: "The role of SNAP29 during epidermal differentiation".

Member of the research staff at the Max Plank Institute for

Experimental Medicine, Göttingen

$2010-2011$

Diploma thesis at the Department of Anthropology at

Georg-August-University of Göttingen, Germany, supervised by Prof.

i.R. Dr. Bernd Hermann with the title: "Die Lichtensteinhöhle als genetisches Archiv - Untersuchungen des Laktosetoleranz- Markers

C/T 13910 an dem bronzezeitlichen Skelettkollektiv."

2005-2011

Study of Biology (Diploma),

Georg-August-University Göttingen, Germany

Major exam subject: Anthropology, Human Genetics and Forensic

Medicine

High School Diploma at the Grotefend-Gymnasium Hann. Münden, Germany 